Supporting Information

\title{
Oxidative Addition of Aryl and Alkyl Halides to a Reduced Iron Pincer Complex
}

\author{
Stephan M. Rummelt, Paul O. Peterson, Hongyu Zhong, Paul J. Chirik* \\ Department of Chemistry, Frick Laboratory \\ Princeton University, Princeton, NJ 08544, USA \\ pchirik@princeton.edu
}

\section{Table of Contents}

General Considerations $\quad$ S2

Preparation of Oxidative Addition Complexes $\quad$ S4

Additional Reactions and Associated NMR data $\quad$ S17

$\begin{array}{ll}\text { Competition Experiments } & \text { S23 }\end{array}$

$\begin{array}{ll}\text { Additional Spectroscopic Data } & \text { S35 }\end{array}$

$\begin{array}{ll}\text { References } & \text { S66 }\end{array}$ 


\section{General Considerations}

All air- and moisture-sensitive manipulations were carried out using vacuum line, Schlenk and cannula techniques or in an MBraun inert atmosphere (nitrogen) dry box unless otherwise noted. All glassware was stored in a pre-heated oven prior to use. The solvents used for air- and moisture-sensitive manipulations were dried and deoxygenated using literature procedures. ${ }^{1}$ All liquid aryl and alkyl halides were dried over $\mathrm{CaH}_{2}$ for 24 hours, degassed via three freeze-pumpthaw cycles, and vacuum distilled prior to glovebox storage. The following compounds were prepared according to literature procedures: $\left(3,5-\mathrm{Me}_{2}-{ }^{\mathrm{Mes}} \mathrm{CNC}\right) \mathrm{Fe}\left(\mathrm{N}_{2}\right)_{2}{ }^{2}$

${ }^{1} \mathrm{H}$ NMR spectra were recorded on either Bruker ADVANCE 300 or 500 spectrophotometers operating at $300.13 \mathrm{MHz}$, and $500.46 \mathrm{MHz}$, respectively. ${ }^{13} \mathrm{C}$ NMR spectra were recorded on either Bruker ADVANCE 300 or 500 spectrometer operating at $75.48 \mathrm{MHz}$ and $125.85 \mathrm{MHz}$, respectively. All ${ }^{1} \mathrm{H}$ and ${ }^{13} \mathrm{C}$ NMR chemical shifts are reported in ppm relative to $\mathrm{SiMe}_{4}$ using the ${ }^{1} \mathrm{H}$ (chloroform-d: $7.26 \mathrm{ppm}$; benzene- $d_{6}: 7.16 \mathrm{ppm}$ ) and ${ }^{13} \mathrm{C}$ (chloroform- $d: 77.16 \mathrm{ppm}$; benzene$d_{6}: 128.06 \mathrm{ppm}$ ) chemical shifts of the solvent as a standard. ${ }^{1} \mathrm{H}$ NMR data for diamagnetic compounds are reported as follows: chemical shift, multiplicity $(s=$ singlet, $d=$ doublet, $t=$ triplet, $q=$ quartet,$p=$ pentet,$b r=$ broad,$m=$ multiplet, $a p p=$ apparent, obsc $=$ obscured), coupling constants $(\mathrm{Hz})$, integration, assignment. ${ }^{1} \mathrm{H}$ NMR data for paramagnetic compounds are reported as follows: chemical shift, integration, peak width at half height $(\mathrm{Hz}) \cdot{ }^{13} \mathrm{C}$ NMR data for diamagnetic compounds are reported as follows: chemical shift, number of protons attached to carbon (e.g. $\mathrm{CH}_{2}$ ), assignment.

Zero-field ${ }^{57} \mathrm{Fe}$ Mössbauer spectra were recorded on a SEE Co. Mössbauer spectrometer (MS4) at $80 \mathrm{~K}$ in constant acceleration mode. ${ }^{57} \mathrm{Co} / \mathrm{Rh}$ was used as the radiation source. WMOSS software $^{2}$ was used for the quantitative evaluation of the spectral parameters (least-squares fitting to Lorentzian peaks). The temperature of the sample was controlled by a Janis Research Co. CCS-850 He/ $\mathrm{N}_{2}$ cryostat within an accuracy of $0.3 \mathrm{~K}$. Isomer shifts were determined relative to airon at $298 \mathrm{~K}$. 
GC analyses were performed using a Shimadzu GC-2010 gas chromatograph equipped with a Shimadzu AOC-20s autosampler and a Shimadzu SHRXI-5MS capillary column $(15 \mathrm{~m} x$ $250 \mu \mathrm{m})$. The instrument was set to an injection volume of $5 \mu \mathrm{L}$, and inlet and detector temperatures of $250^{\circ} \mathrm{C}$ and $275^{\circ} \mathrm{C}$, respectively. UHP-grade S3 helium was used as carrier gas with a flow rate of $1.82 \mathrm{~mL} / \mathrm{min}$. The temperature program used was as follows: $60^{\circ} \mathrm{C}$, isothermal $1 \mathrm{~min} ; 15^{\circ} \mathrm{C} / \mathrm{min}$ to $250^{\circ} \mathrm{C}$, isothermal $2 \mathrm{~min}$. GC-MS analyses were performed on an Agilent 7890A gas chromatograph equipped with an Agilent 5975 mass selective detector (electrospray ionization method), using a RESTEC Rtx®-35MS, Crossbond® $35 \%$ diphenyl-65\% dimethylpolysiloxane column ( $30 \mathrm{~m}, 0.32 \mathrm{mmID}, 0.5 \mu \mathrm{m} \mathrm{df})$. The instrument was set to an injection volume of $0.5 \mu \mathrm{L}$ (sample dissolved in dichloromethane) with inlet and detector temperatures of $250{ }^{\circ} \mathrm{C}$. UHP-grade S3 helium was used as carrier gas with a flow rate of $1.82 \mathrm{~mL} / \mathrm{min}$. The temperature program was as follows: $30^{\circ} \mathrm{C}, 5^{\circ} \mathrm{C} / \mathrm{min}$ to $50^{\circ} \mathrm{C}$, isothermal $5 \mathrm{~min}, 10^{\circ} \mathrm{C} / \mathrm{min}$ to 225 ${ }^{\circ} \mathrm{C}$.

Elemental analyses were performed at Robinson Microlit Laboratories, Inc., in Ledgewood, NJ. Infrared spectroscopy was conducted on a Thermo-Nicolet iS10 FT-IR spectrometer calibrated with a polystyrene standard.

Single crystals suitable for X-ray diffraction were coated with polyisobutylene oil in a drybox, transferred to a nylon loop and then quickly transferred to the goniometer head of a Bruker SMART APEX DUO diffractometer equipped with a molybdenum X-ray tube $(\lambda=0.71073 \AA)$ and a Cu X-ray tube $(\lambda=1.54178 \AA)$. Preliminary data revealed the crystal system. The data collection strategy was optimized for completeness and redundancy using the Bruker COSMO software suite. The space group was identified, and the data were processed using the Bruker SAINT+ program and corrected for absorption using SADABS. The structures were solved using direct methods (SHELXS) completed by subsequent Fourier synthesis and refined by full-matrix leastsquares procedures. 


\section{Preparation of Oxidative Addition Complexes.}
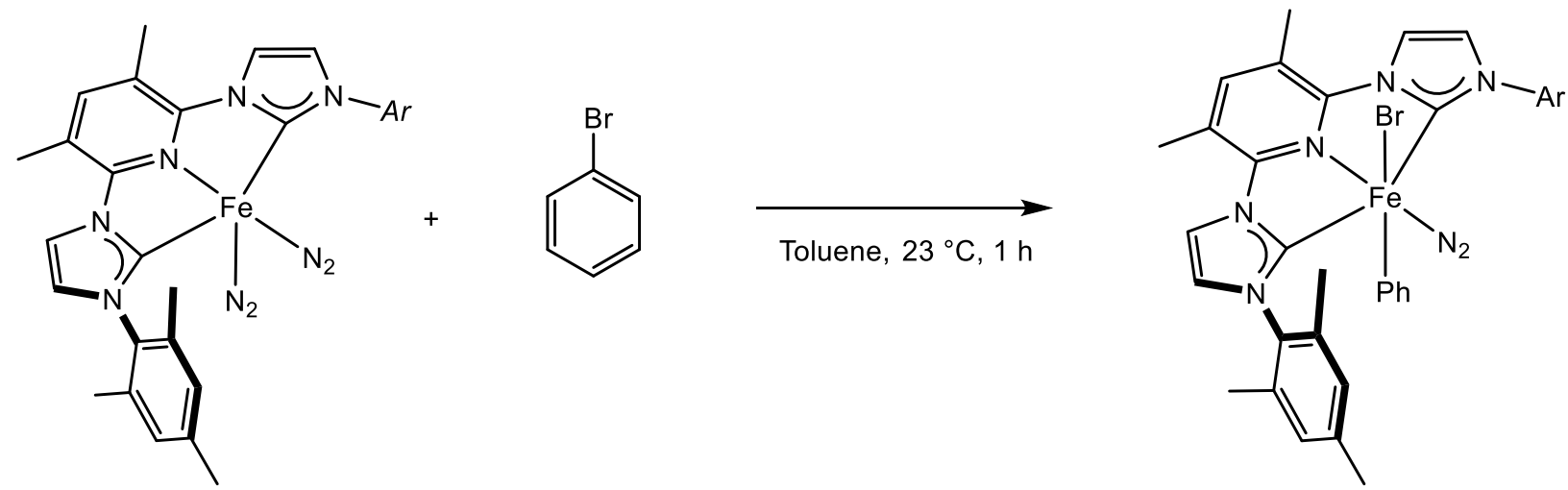

Preparation of (3,5-Me $\left.{ }_{2}{ }^{\mathrm{Mes}} \mathrm{CNC}\right) \mathrm{FePh}\left(\mathrm{N}_{2}\right) \mathrm{Br}$. In a $\mathrm{N}_{2}$-filled glovebox a $20 \mathrm{~mL}$ scintillation vial was charged with (3,5-Me $\left.{ }_{2}^{\mathrm{Mes}} \mathrm{CNC}\right) \mathrm{Fe}\left(\mathrm{N}_{2}\right)_{2}(137 \mathrm{mg}, 0.23 \mathrm{mmol}, 1.0$ equiv) and toluene (ca. $3 \mathrm{~mL}$ ). $\mathrm{PhBr}(27 \mu \mathrm{L}, 0.26 \mathrm{mmol}, 1.1$ equiv) was added at $\mathrm{RT}$ and the reaction mixture was stirred for $1 \mathrm{~h}$. Pentane (ca. $3 \mathrm{~mL}$ ) was added and the resulting suspension was filtered and the solid was washed with pentane $(3 \times 3 \mathrm{~mL})$ and dried under vacuum to yield $131 \mathrm{mg}(79 \%$ yield $)$ of $(3,5-$ $\left.\mathrm{Me}_{2}{ }^{\mathrm{Mes}} \mathrm{CNC}\right) \mathrm{FePh}\left(\mathrm{N}_{2}\right) \mathrm{Br}$ as a brown-red solid. Single crystals of poor quality suitable for X-ray diffraction were grown from an oversaturated solution in $\mathrm{C}_{6} \mathrm{H}_{6}$ at $\mathrm{RT}$. Anal Calcd for $\mathrm{C}_{37} \mathrm{H}_{38} \mathrm{BrFeN}_{7}$ : $\mathrm{C}, 62.02 ; \mathrm{H}, 5.35 ; \mathrm{N}, 13.68$. Found: $\mathrm{C}, 61.75 ; \mathrm{H}, 5.51 ; \mathrm{N}, 12.98 . \mathrm{IR}\left(\mathrm{C}_{6} \mathrm{D}_{6}\right): \mathrm{v}_{\mathrm{NN}}=2139 \mathrm{~cm}^{-1} .{ }^{1} \mathrm{H}$ $\operatorname{NMR}\left(500 \mathrm{MHz}, \mathrm{C}_{6} \mathrm{D}_{6}, 2{ }^{\circ} \mathrm{O}\right)$ : $\delta 7.29(\mathrm{~d}, J=2.1 \mathrm{~Hz}, 2 \mathrm{H}, 4 / 5$-imidazolylidene $H), 6.84(\mathrm{~s}, 2 \mathrm{H}, \mathrm{Ar}$ H), $6.74(\mathrm{~s}, 2 \mathrm{H}, \mathrm{Ar} H), 6.72-6.69(\mathrm{~m}, 3 \mathrm{H}, \mathrm{Ph} H), 6.46-6.40(\mathrm{~m}, 2 \mathrm{H}, \mathrm{Ph} H), 6.42(\mathrm{~s}, 1 \mathrm{H}, \mathrm{p}-\mathrm{pyr}$ H), $6.15\left(\mathrm{~d}, J=2.0 \mathrm{~Hz}, 2 \mathrm{H}, 4 / 5\right.$-imidazolylidene H), 2.91 (s, 6H, Ar- $\mathrm{CH}_{3}$ ), 2.02 (s, 6H, Ar- $\mathrm{CH}_{3}$ ), 1.77 (s, 6H, m-pyr- $\left.\left(\mathrm{CH}_{3}\right)_{2}\right), 1.43\left(\mathrm{~s}, 6 \mathrm{H}, \mathrm{Ar}-\mathrm{CH}_{3}\right) .{ }^{13} \mathrm{C}\left\{{ }^{1} \mathrm{H}\right\} \mathrm{NMR}\left(201 \mathrm{MHz}, \mathrm{C}_{6} \mathrm{D}_{6}, 25{ }^{\circ} \mathrm{C}\right): \delta 219.4$ (2-imidazolylidene C), 165.5 (1-Ph C), 152.2 (o-pyr C), 143.2 (p-pyr C), 138.9 (Ar C), 138.3 ( $\operatorname{Ar}$ C), $138.1(\operatorname{Ph} C), 136.6(\operatorname{Ar} C), 136.1(\operatorname{Ar} C), 129.6(3-\operatorname{Ar} C), 128.7(3-\operatorname{Ar} C), 125.6(\operatorname{Ph} C), 123.9$ (4/5-imidazolylidene C), 120.1 ( $\mathrm{Ph} \mathrm{C),} 118.1$ (4/5-imidazolylidene C), 114.2 (m-pyr C), 21.1 (Ar$\left.\mathrm{CH}_{3}\right), 20.5\left(\mathrm{Ar}-\mathrm{CH}_{3}\right), 18.2\left(\mathrm{~m}-\mathrm{pyr}-\left(\mathrm{CH}_{3}\right)_{2}\right), 17.9\left(\mathrm{Ar}-\mathrm{CH}_{3}\right)$. 

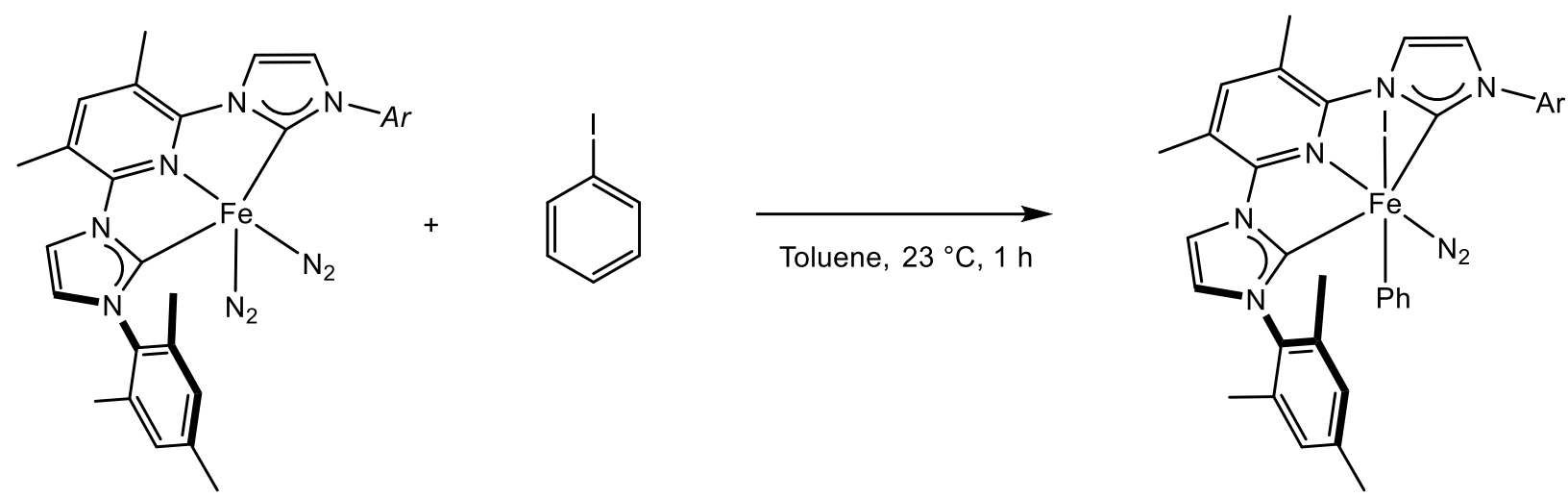

Preparation of $\left(3,5-\mathrm{Me}_{2}{ }^{\mathrm{Mes}} \mathrm{CNC}\right) \mathrm{FePh}\left(\mathrm{N}_{2}\right)$ I. In a $\mathrm{N}_{2}$-filled glovebox a $20 \mathrm{~mL}$ scintillation vial was charged with (3,5-Me $\left.{ }^{\mathrm{Mes}} \mathrm{CNC}\right) \mathrm{Fe}\left(\mathrm{N}_{2}\right)_{2}(100 \mathrm{mg}, 0.17 \mathrm{mmol}, 1.0$ equiv) and toluene (ca. $3 \mathrm{~mL}$ ). $\mathrm{Phl}(21 \mu \mathrm{L}, 0.19 \mathrm{mmol}, 1.1$ equiv) was added at RT and the reaction mixture was stirred for $1 \mathrm{~h}$. Pentane (ca. $3 \mathrm{~mL}$ ) was added and the resulting suspension was filtered and the solid was washed with pentane $(3 \times 3 \mathrm{~mL})$ and dried under vacuum to yield $77 \mathrm{mg}(59 \%$ yield) of $(3,5-$ $\left.\mathrm{Me}_{2}{ }^{\mathrm{Mes}} \mathrm{CNC}\right) \mathrm{FePh}\left(\mathrm{N}_{2}\right)$ I as a brown-red solid. Single crystals suitable for X-ray diffraction were grown through slow evaporation of pentane into a concentrated toluene solution at $-35{ }^{\circ} \mathrm{C}$.

Anal Calcd for $\mathrm{C}_{37} \mathrm{H}_{38} \mathrm{FelN}_{7}$ : C, 58.21; $\mathrm{H}, 5.02 ; \mathrm{N}, 12.84$. Found: $\mathrm{C}, 58.56 ; \mathrm{H}, 5.31 ; \mathrm{N}, 12.46$. IR $\left(\mathrm{C}_{6} \mathrm{D}_{6}\right): \mathrm{v}_{\mathrm{NN}}=2134 \mathrm{~cm}^{-1} .{ }^{1} \mathrm{H}$ NMR $\left(400 \mathrm{MHz}, \mathrm{C}_{6} \mathrm{D}_{6}, 25 \stackrel{\circ}{\circ} \mathrm{C}\right): \delta 7.36(\mathrm{~d}, J=2.2 \mathrm{~Hz}, 2 \mathrm{H}, 4 / 5-$ imidazolylidene $H$ ), $6.85-6.81(\mathrm{~m}, 2 \mathrm{H}, \mathrm{Ar} H), 6.74-6.69(\mathrm{~m}, 5 \mathrm{H}, \mathrm{Ph} H$ and $\mathrm{Ar} H), 6.48(\mathrm{~s}, 1 \mathrm{H}$, p-pyr H), $6.39-6.30(\mathrm{~m}, 2 \mathrm{H}, \mathrm{Ph} H$ ), $6.16(\mathrm{~d}, J=2.2 \mathrm{~Hz}, 2 \mathrm{H}, 4 / 5$-imidazolylidene $H$ ), 2.94 (s, 6H, Ar- $\left.\mathrm{CH}_{3}\right), 2.00$ (s, 6H, Ar- $\left.\mathrm{CH}_{3}\right), 1.84$ (s, 6H, m-pyr- $\left.\left(\mathrm{CH}_{3}\right)_{2}\right), 1.33$ (s, 6H, Ar- $\left.\mathrm{CH}_{3}\right) .{ }^{13} \mathrm{C}\left\{{ }^{1} \mathrm{H}\right\} \mathrm{NMR}(101$ MHz, $\mathrm{C}_{6} \mathrm{D}_{6}, 25^{\circ} \mathrm{C}$ ): $\delta 219.6$ (2-imidazolylidene C), 166.7 (1-Ph C), 151.9 (o-pyr C), 143.3 (p-pyr C), $138.9(\operatorname{Ar} C), 138.2(\operatorname{Ar} C), 137.4(\operatorname{Ph} C), 136.9(\operatorname{Ar} C), 136.0(\operatorname{Ar} C), 129.6(3-\operatorname{Ar} C), 128.8(3-$ $\operatorname{Ar} C$ ), 125.8 ( $P h C), 124.4$ (4/5-imidazolylidene C), 120.2 ( $\mathrm{Ph} C$ ), 118.0 (4/5-imidazolylidene C), $114.2(\mathrm{~m}-\mathrm{pyr} \mathrm{C}), 21.5\left(\mathrm{Ar}-\mathrm{CH}_{3}\right), 21.1\left(\mathrm{Ar}-\mathrm{CH}_{3}\right), 18.2\left(\mathrm{~m}-\mathrm{pyr}-\left(\mathrm{CH}_{3}\right)_{2}\right), 17.9\left(\mathrm{Ar}-\mathrm{CH}_{3}\right)$. 

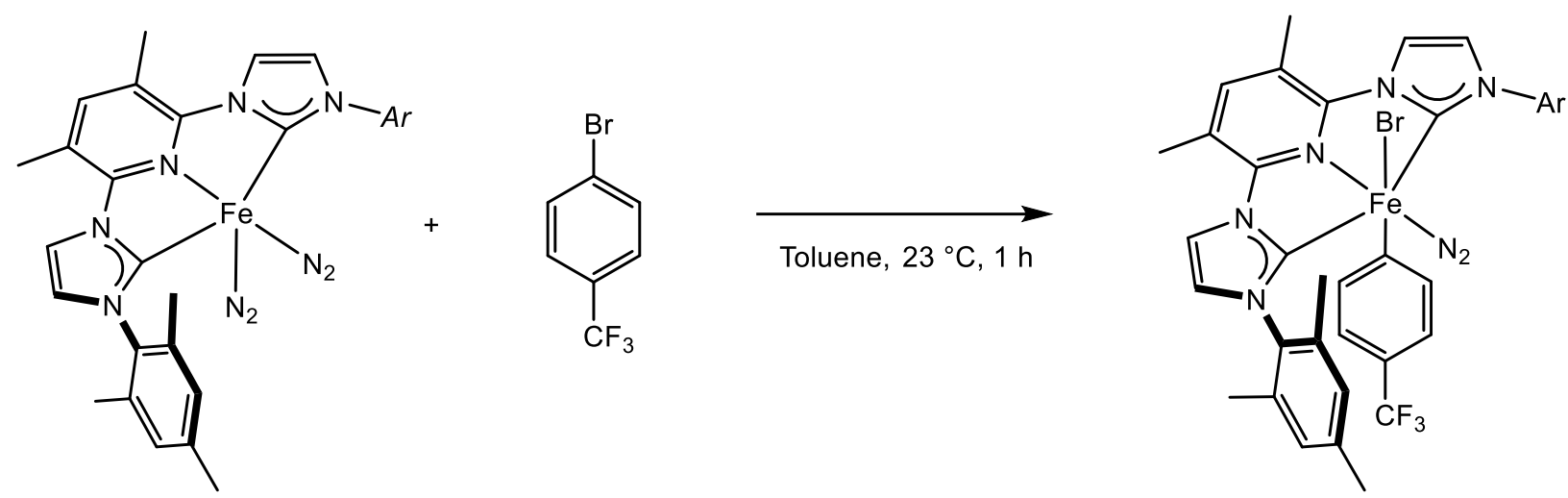

Preparation of $\left(3,5-\mathrm{Me}_{2}{ }^{\mathrm{Mes}} \mathbf{C N C}\right) \mathrm{Fe}\left(\mathrm{C}_{6} \mathrm{H}_{4} \mathbf{C F}_{3}\right)\left(\mathbf{N}_{2}\right) \mathrm{Br}$. In a $\mathrm{N}_{2}$-filled glovebox a $20 \mathrm{~mL}$ scintillation vial was charged with $\left(3,5-\mathrm{Me}_{2}{ }^{\mathrm{Mes}} \mathrm{CNC}\right) \mathrm{Fe}\left(\mathrm{N}_{2}\right)_{2}(40 \mathrm{mg}, 0.068 \mathrm{mmol}, 1.0$ equiv) and toluene (ca. $2 \mathrm{~mL}$ ). 1-bromo-4-(trifluoromethyl)benzene (10.5 $\mathrm{LL}, 0.075 \mathrm{mmol}, 1.1$ equiv) was added at RT and the reaction mixture was stirred for $1 \mathrm{~h}$. Pentane (ca. $6 \mathrm{~mL}$ ) was added and the resulting suspension was filtered and the solid was washed with pentane $(3 \times 3 \mathrm{~mL})$ and dried under vacuum to yield $39 \mathrm{mg}\left(73 \%\right.$ yield) of $\left(3,5-\mathrm{Me}_{2}{ }^{\mathrm{Mes}} \mathrm{CNC}\right) \mathrm{Fe}\left(\mathrm{C}_{6} \mathrm{H}_{4} \mathrm{CF}_{3}\right)\left(\mathrm{N}_{2}\right) \mathrm{Br}$ as a brown-red solid. Anal Calcd for $\mathrm{C}_{38} \mathrm{H}_{37} \mathrm{BrF}_{3} \mathrm{FeN}_{7}$ : C, 58.18; $\mathrm{H}, 4.75 ; \mathrm{N}, 12.50$. Found: $\mathrm{C}, 57.93 ; \mathrm{H}, 5.16 ; \mathrm{N}, 12.82$. IR $\left(\mathrm{C}_{6} \mathrm{D}_{6}\right): \mathrm{v}_{\mathrm{NN}}=2141 \mathrm{~cm}^{-1} .{ }^{1} \mathrm{H}$ NMR $\left(500 \mathrm{MHz}, \mathrm{C}_{6} \mathrm{D}_{6}, 2{ }^{\circ} \mathrm{C}\right): \delta 7.29(\mathrm{~s}, 2 \mathrm{H}, 4 / 5$-imidazolylidene H), $6.86\left(\mathrm{~d}, J=8.0 \mathrm{~Hz}, 2 \mathrm{H}, \mathrm{C}_{6} H_{4} \mathrm{CF}_{3}\right), 6.82(\mathrm{~s}, 2 \mathrm{H}, \operatorname{Ar} H), 6.66(\mathrm{~s}, 2 \mathrm{H}, \operatorname{Ar} H), 6.46(\mathrm{~d}, J=7.7 \mathrm{~Hz}$, 2H, $\mathrm{C}_{6} \mathrm{H}_{4} \mathrm{CF}_{3}$ ), 6.43 (s, 1H, p-pyr H), 6.09 (s, 2H, 4/5-imidazolylidene $H$ ), 2.85 (s, 6H, Ar- $\mathrm{CH}_{3}$ ), 2.01 (s, 6H, Ar- $\left.\mathrm{CH}_{3}\right), 1.78$ (s, 6H, m-pyr- $\left.\left(\mathrm{CH}_{3}\right)_{2}\right), 1.26$ (s, 6H, Ar- $\left.\mathrm{CH}_{3}\right) .{ }^{13} \mathrm{C}\left\{{ }^{1} \mathrm{H}\right\} \mathrm{NMR}(126 \mathrm{MHz}$, $\mathrm{C}_{6} \mathrm{D}_{6}, 25 \stackrel{\circ}{\circ}$ ) : $\delta 217.8$ (2-imidazolylidene C), $178.9\left(1-\mathrm{C}_{6} \mathrm{H}_{4} \mathrm{CF}_{3} \mathrm{C}\right.$ ), 152.1 (o-pyr C), 144.1 (p-pyr C), $139.1(\operatorname{Ar} C), 138.1\left(\mathrm{C}_{6} \mathrm{H}_{4} \mathrm{CF}_{3} \mathrm{CH}\right.$ and $\left.\operatorname{Ar} C\right), 136.3(\operatorname{Ar} C), 135.8(\operatorname{Ar} C), 129.6(3-\operatorname{Ar} C), 128.8$ (3- $\operatorname{Ar} C), 124.3$ (4/5-imidazolylidene $C$ ), $120.6\left(\mathrm{C}_{6} \mathrm{H}_{4} \mathrm{CF}_{3} \mathrm{CH}\right), 118.3$ (4/5-imidazolylidene $\left.C\right), 114.6$ (m-pyr C), $21.01\left(\mathrm{Ar}-\mathrm{CH}_{3}\right), 20.3\left(\mathrm{Ar}-\mathrm{CH}_{3}\right), 18.4\left(\mathrm{~m}-\mathrm{pyr}-\left(\mathrm{CH}_{3}\right)_{2}\right), 17.6\left(\mathrm{Ar}-\mathrm{CH}_{3}\right) .\left(\mathrm{C}-\mathrm{CF}_{3}\right.$ and $\mathrm{C}-\mathrm{CF}_{3}$ not located). 

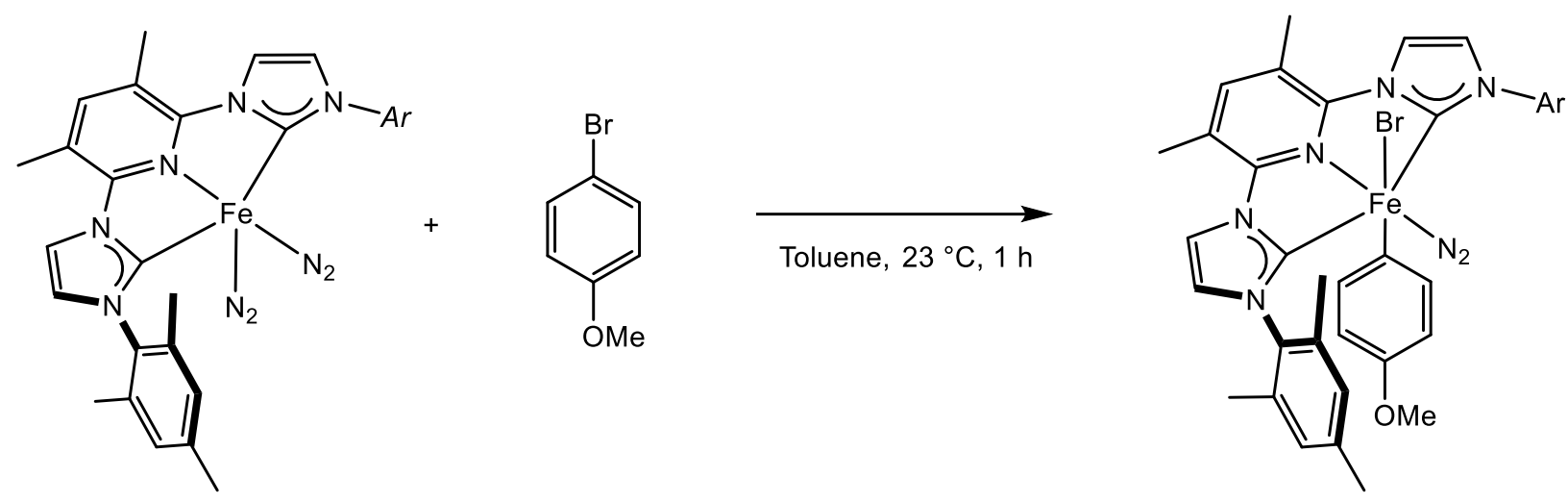

Preparation of (3,5-Me $\left.{ }_{2}{ }^{\mathrm{Mes}} \mathrm{CNC}\right) \mathrm{Fe}\left(\mathrm{C}_{6} \mathrm{H}_{4} \mathrm{OMe}\right)\left(\mathrm{N}_{2}\right) \mathrm{Br}$. In a $\mathrm{N}_{2}$-filled glovebox a $20 \mathrm{~mL}$ scintillation vial was charged with $\left(3,5-\mathrm{Me}_{2}{ }^{\mathrm{Mes}} \mathrm{CNC}\right) \mathrm{Fe}\left(\mathrm{N}_{2}\right)_{2}(40 \mathrm{mg}, 0.068 \mathrm{mmol}, 1.0$ equiv) and toluene (ca. $2 \mathrm{~mL})$. 1-bromo-4-methoxybenzene $(9.4 \mu \mathrm{L}, 0.075 \mathrm{mmol}, 1.1$ equiv) was added at RT and the reaction mixture was stirred for $1 \mathrm{~h}$. Pentane (ca. $6 \mathrm{~mL}$ ) was added and the resulting suspension was filtered and the solid was washed with pentane $(3 \times 3 \mathrm{~mL})$ and dried under vacuum to yield $34 \mathrm{mg}(67 \%$ yield $)$ of $\left(3,5-\mathrm{Me}_{2}{ }^{\mathrm{Mes}} \mathrm{CNC}\right) \mathrm{Fe}\left(\mathrm{C}_{6} \mathrm{H}_{4} \mathrm{OMe}\right)\left(\mathrm{N}_{2}\right) \mathrm{Br}$ as a brown-red solid.

Anal Calcd for $\mathrm{C}_{38} \mathrm{H}_{40} \mathrm{BrFeN} 7 \mathrm{O}: \mathrm{C}, 61.14 ; \mathrm{H}, 5.40 ; \mathrm{N}, 13.13$. Found: $\mathrm{C}, 60.88 ; \mathrm{H}, 5.71 ; \mathrm{N}, 13.11$. IR $\left(\mathrm{C}_{6} \mathrm{D}_{6}\right): \mathrm{v}_{\mathrm{NN}}=2136,2117 \mathrm{~cm}^{-1} .{ }^{1} \mathrm{H}$ NMR $\left(500 \mathrm{MHz}, \mathrm{C}_{6} \mathrm{D}_{6}, 25 \stackrel{\circ}{\circ} \mathrm{C}\right): \delta 7.31(\mathrm{~s}, 2 \mathrm{H}, 4 / 5-$ imidazolylidene H), $6.84(\mathrm{~s}, 2 \mathrm{H}, \operatorname{Ar} H), 6.73(\mathrm{~s}, 2 \mathrm{H}, \operatorname{Ar} H), 6.46\left(\mathrm{~d}, J=8.1 \mathrm{~Hz}, 2 \mathrm{H}, \mathrm{C}_{6} H_{4} \mathrm{OCH}_{3}\right)$, $6.43\left(\mathrm{~s}, 1 \mathrm{H}, \mathrm{p}\right.$-pyr $H$ ), $6.23\left(\mathrm{~d}, J=8.0 \mathrm{~Hz}, 2 \mathrm{H}, \mathrm{C}_{6} \mathrm{H}_{4} \mathrm{OCH}_{3}\right), 6.17$ (s, 2H, 4/5-imidazolylidene $H$ ), $3.34\left(\mathrm{~s}, 3 \mathrm{H}, \mathrm{C}_{6} \mathrm{H}_{4} \mathrm{OCH}_{3}\right), 2.91$ (s, 6H, Ar- $\mathrm{CH}_{3}$ ), 2.03 (s, 6H, Ar- $\left.\mathrm{CH}_{3}\right), 1.80$ (s, 6H, m-pyr- $\left(\mathrm{CH}_{3}\right)_{2}$ ), 1.45 (s, 6H, Ar- $\left.\mathrm{CH}_{3}\right) .{ }^{13} \mathrm{C}\left\{{ }^{1} \mathrm{H}\right\} \mathrm{NMR}\left(126 \mathrm{MHz}, \mathrm{C}_{6} \mathrm{D}_{6}, 25 \stackrel{\circ}{\circ} \mathrm{C}\right): \delta 219.9$ (2-imidazolylidene $\mathrm{C}$ ), 156.0 (4- $\mathrm{C}_{6} \mathrm{H}_{4} \mathrm{OCH}_{3}$ C), 152.3 (o-pyr C), $150.3\left(1-\mathrm{C}_{6} \mathrm{H}_{4} \mathrm{OCH}_{3}\right.$ C), 143.3 (p-pyr C), 138.8 (Ar C), 138.3 $(\operatorname{Ar} C), 137.6\left(\mathrm{C}_{6} \mathrm{H}_{4} \mathrm{OCH}_{3} C\right), 136.6(\operatorname{Ar} C), 136.2(\operatorname{Ar} C), 129.6(3-\operatorname{Ar} C), 128.7(3-\operatorname{Ar} C), 124.0$ (4/5-imidazolylidene C), 118.2 (4/5-imidazolylidene $C$ ), 114.2 (m-pyr $C$ ), $112.6\left(\mathrm{C}_{6} \mathrm{H}_{4} \mathrm{OCH}_{3} C\right.$ ), 54.8 $\left(\mathrm{C}_{6} \mathrm{H}_{4} \mathrm{OCH}_{3}\right), 21.1\left(\mathrm{Ar}-\mathrm{CH}_{3}\right), 20.5\left(\mathrm{Ar}-\mathrm{CH}_{3}\right), 18.3\left(\mathrm{~m}-\mathrm{pyr}-\left(\mathrm{CH}_{3}\right)_{2}\right), 18.0\left(\mathrm{Ar}-\mathrm{CH}_{3}\right)$. 

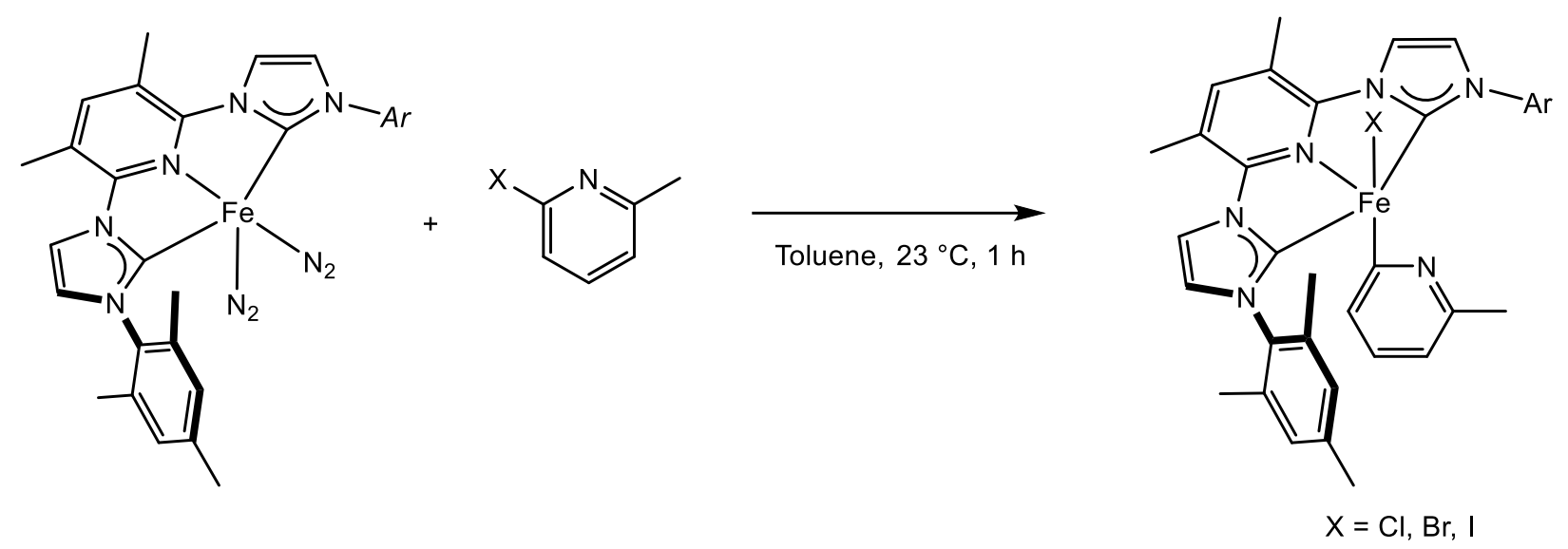

Preparation of (3,5-Me $\left.{ }_{2}{ }^{-M e s} \mathrm{CNC}\right) \mathrm{Fe}(6-M e$ pyridine-2-yl)I. In the glovebox, a $20 \mathrm{~mL}$ scintillation vial was charged with 3,5-Me ${ }_{2}{ }^{-M e s} \mathrm{CNCFe}\left(\mathrm{N}_{2}\right)_{2}$ (37 mg, $0.06 \mathrm{mmol}, 1.0$ equiv.), toluene (ca. $3 \mathrm{~mL}$ ), and a Teflon-coated stir bar. To the stirred solution was added 2-iodo-6-methylpyridine $(0.07$ mmol, 1.1 equiv.) via syringe. Upon addition, the solution turned from brown to green. The solution was then stirred at room temperature for 90 minutes. Pentane $(5 \mathrm{~mL})$ was added, resulting in precipitation of a green solid. The precipitate was isolated via filtration and washed with $3 \times 3 \mathrm{~mL}$ pentane to yield $32 \mathrm{mg}$ (68\% yield) of a green solid identified as $\left(3,5-\mathrm{Me}_{2}{ }^{-\mathrm{Mes}} \mathrm{CNC}\right) \mathrm{Fe}(6-\mathrm{Me}$ pyridine-2-yl)I. Anal Calcd for $\mathrm{C}_{37} \mathrm{H}_{39} \mathrm{FelN}_{6}$ : C, 59.21; H, 5.24; N, 11.20. Found: C, 58.85; H, 4.90; $\mathrm{N}, 10.93 .{ }^{1} \mathrm{H}$ NMR $\left(400 \mathrm{MHz}, \mathrm{C}_{6} \mathrm{D}_{6}, 25^{\circ} \mathrm{C}\right): \delta 7.64$ (s, $2 \mathrm{H}, 4 / 5$-imidazolylidene $H$ ), 6.73 (s, 2H, $\mathrm{Ar}$ H), $6.42(\mathrm{~s}, 2 \mathrm{H}, \mathrm{Ar} H), 6.28(\mathrm{~m}, 4 \mathrm{H}), 6.10(\mathrm{~d}, 7.1 \mathrm{~Hz}, 1 \mathrm{H}$, picoline H), $5.45(\mathrm{~d}, 7.4 \mathrm{~Hz}, 1 \mathrm{H}$, picoline H), $2.99\left(\mathrm{~s}, 6 \mathrm{H}, \mathrm{Ar}-\mathrm{CH}_{3}\right), 2.10\left(\mathrm{~s}, 6 \mathrm{H}, \mathrm{Ar}-\mathrm{CH}_{3}\right), 2.06\left(\mathrm{~s}, 6 \mathrm{H}, \mathrm{m}-\mathrm{pyr}-\left(\mathrm{CH}_{3}\right)_{2}\right), 1.66\left(\mathrm{~s}, 3 \mathrm{H}, \mathrm{CH}_{3} \mathrm{C}_{5} \mathrm{H}_{3} \mathrm{~N}\right)$ $1.56\left(\mathrm{~s}, 6 \mathrm{H}, \mathrm{Ar}-\mathrm{CH}_{3}\right)$.

Preparation of $\left(3,5-\mathrm{Me}_{2}{ }^{-\mathrm{Mes}} \mathrm{CNC}\right) \mathrm{Fe}\left(6-\mathrm{Me}\right.$ pyridine-2-yl)Br. The compound (3,5-Me $\mathrm{Me}^{-}$ $\left.{ }^{\mathrm{Mes}} \mathrm{CNC}\right) \mathrm{Fe}(6-\mathrm{Me}$ pyridine-2-yl) $\mathrm{Br}$ was prepared in an analogous fashion to (3,5-Me-

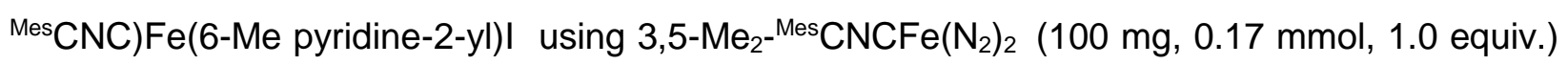
and 2-bromo-6-methylpyridine ( $0.19 \mathrm{mmol}, 1.1$ equiv.). This procedure yielded $95 \mathrm{mg}$ ( $79 \%$ yield) of a green solid identified as (3,5-Me $\left.{ }^{-}{ }^{M e s} \mathrm{CNC}\right) \mathrm{Fe}\left(6-\mathrm{Me}\right.$ pyridine-2-y) Br. ${ }^{1} \mathrm{H} \mathrm{NMR}\left(400 \mathrm{MHz}, \mathrm{C}_{6} \mathrm{D}_{6}\right.$, $\left.25^{\circ} \mathrm{C}\right):{ }^{1} \mathrm{H}$ NMR $\left(400 \mathrm{MHz}, \mathrm{C}_{6} \mathrm{D}_{6}, 25^{\circ} \mathrm{C}\right): \delta 7.60$ (s, 2H, 4/5-imidazolylidene $H$ ), 6.78 (s, 2H, $\mathrm{Ar} \mathrm{H}$ ), $6.48(\mathrm{~s}, 2 \mathrm{H}, \mathrm{Ar} H), 6.31(\mathrm{~m}, 3 \mathrm{H}), 6.24(\mathrm{~s}, 1 \mathrm{H}, \mathrm{p}-\mathrm{pyr} H), 6.04(\mathrm{~d}, 7.1 \mathrm{~Hz}, 1 \mathrm{H}$, picoline $H), 5.55(\mathrm{~d}$, 
$7.4 \mathrm{~Hz}, 1 \mathrm{H}$, picoline H), 2.97 (s, 6H, Ar- $\left.\mathrm{CH}_{3}\right), 2.13\left(\mathrm{~s}, 6 \mathrm{H}, \mathrm{Ar}-\mathrm{CH}_{3}\right), 2.04$ (s, 6H, m-pyr- $\left.\left(\mathrm{CH}_{3}\right)_{2}\right)$, $1.60\left(\mathrm{~s}, 3 \mathrm{H}, \mathrm{CH}_{3} \mathrm{C}_{5} \mathrm{H}_{3} \mathrm{~N}\right) 1.56\left(\mathrm{~s}, 6 \mathrm{H}, \mathrm{Ar}-\mathrm{CH}_{3}\right)$.

Preparation of $\left(3,5-\mathrm{Me}_{2}{ }^{-}{ }^{\mathrm{Mes}} \mathrm{CNC}\right) \mathrm{Fe}(6-\mathrm{Me}$ pyridine-2-yl)Cl. The compound (3,5-Me$\left.{ }^{\mathrm{Mes}} \mathrm{CNC}\right) \mathrm{Fe}(6-\mathrm{Me}$ pyridine-2-yl)Cl was prepared in an analogous fashion to (3,5-Me$\left.{ }^{\mathrm{Mes}} \mathrm{CNC}\right) \mathrm{Fe}\left(6-\mathrm{Me}\right.$ pyridine-2-yl)I using 3,5-Me ${ }_{2}{ }^{-}{ }^{\mathrm{Mes}} \mathrm{CNCFe}\left(\mathrm{N}_{2}\right)_{2}(30 \mathrm{mg}, 0.05 \mathrm{mmol}, 1.0$ equiv.) and 2-chloro-6-methylpyridine ( $0.06 \mathrm{mmol}, 1.1$ equiv.). This procedure yielded $20 \mathrm{mg}$ (59\% yield) of a green solid identified as (3,5-Me $\left.{ }^{-}{ }^{-}{ }^{M e s} \mathrm{CNC}\right) \mathrm{Fe}\left(6-\mathrm{Me}\right.$ pyridine-2-yl) $\mathrm{Cl}$. ${ }^{1} \mathrm{H} \mathrm{NMR}\left(400 \mathrm{MHz}, \mathrm{C}_{6} \mathrm{D}_{6}\right.$, $\left.25^{\circ} \mathrm{C}\right):{ }^{1} \mathrm{H}$ NMR (400 MHz, $\left.\mathrm{C}_{6} \mathrm{D}_{6}, 25^{\circ} \mathrm{C}\right): \delta 7.59$ (s, 2H, 4/5-imidazolylidene H), 6.80 (s, 2H, Ar H), $6.68(\mathrm{~s}, 1 \mathrm{H}, \operatorname{Ar} H), 6.51(\mathrm{~s}, 2 \mathrm{H}, \operatorname{Ar} H), 6.32(\mathrm{~m}, 3 \mathrm{H}), 6.23(\mathrm{~s}, 1 \mathrm{H}, \mathrm{p}-\mathrm{pyr} H), 6.00(\mathrm{~d}, 7.1 \mathrm{~Hz}, 1 \mathrm{H}$, picoline H), $5.60\left(\mathrm{~d}, 7.4 \mathrm{~Hz}, 1 \mathrm{H}\right.$, picoline H), 2.95 (s, 6H, Ar- $\left.\mathrm{CH}_{3}\right), 2.14\left(\mathrm{~s}, 6 \mathrm{H}, \mathrm{Ar}-\mathrm{CH}_{3}\right), 2.04$ (s, $6 \mathrm{H}, \mathrm{m}$-pyr- $\left(\mathrm{CH}_{3}\right)_{2}$ ), 1.56 (overlapping, $9 \mathrm{H}, \mathrm{Ar}-\mathrm{CH}_{3}$ and $\mathrm{CH}_{3} \mathrm{C}_{5} \mathrm{H}_{3} \mathrm{~N}$ ).
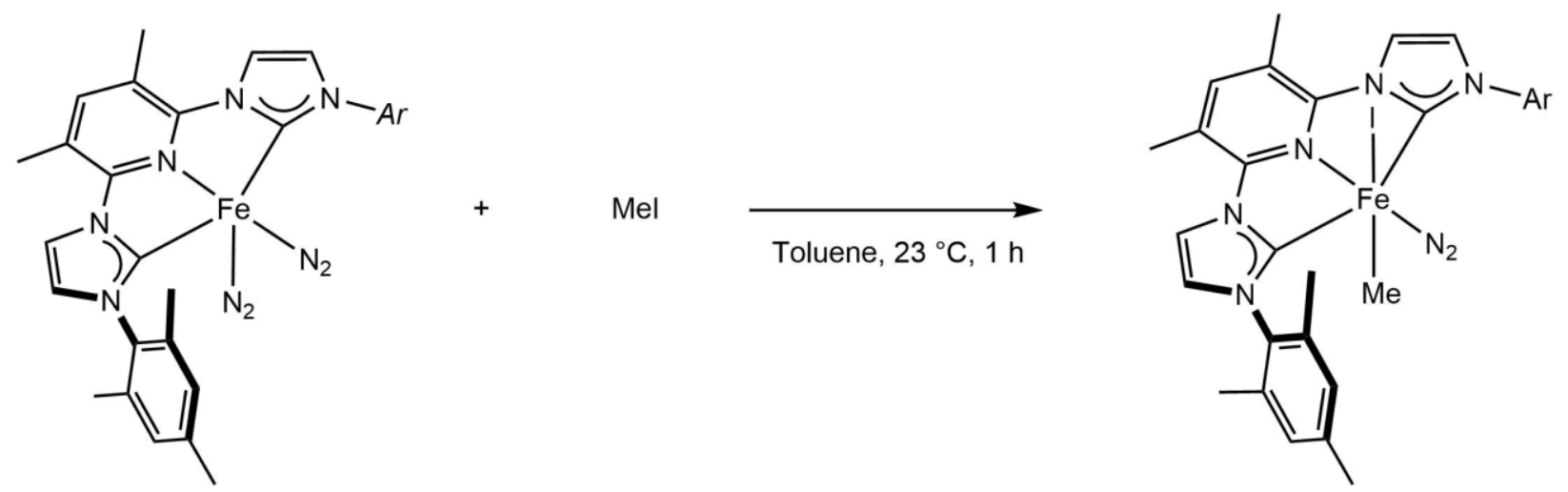

Preparation of $\left(3,5-\mathrm{Me}_{2}{ }^{-\mathrm{Mes}} \mathrm{CNC}\right) \mathrm{Fe}(\mathrm{Me})\left(\mathrm{N}_{2}\right)(\mathrm{I})$. A Schlenk tube was charged with 3,5- $\mathrm{Me}_{2-}$ ${ }^{\text {Mes }} \mathrm{CNCFe}\left(\mathrm{N}_{2}\right)_{2}(200 \mathrm{mg}, 0.34 \mathrm{mmol}, 1.0$ equiv.), toluene (ca. $5 \mathrm{~mL}$ ), and a Teflon-coated stir bar. On the vacuum line, the Schlenk tube was degassed and methyl iodide $(0.37 \mathrm{mmol}, 1.1$ equiv. $)$ was added via calibrated gas bulb at $-196{ }^{\circ} \mathrm{C}$. The solution was then thawed and allowed to stir at room temperature. Upon warming, the solution turned from brown to green. After stirring $1 \mathrm{~h}$, the Schlenk tube was brought into the glovebox. Pentane $(15 \mathrm{~mL})$ was added, resulting in precipitation of a green solid. The precipitate was isolated via filtration and washed with $3 \times 3 \mathrm{~mL}$ 


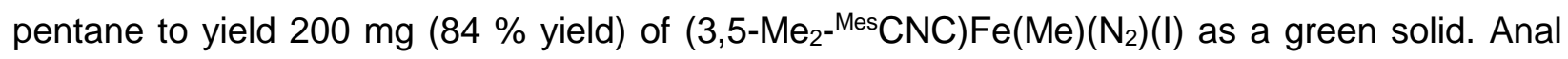
Calcd for $\mathrm{C}_{32} \mathrm{H}_{36} \mathrm{FeIN}_{7}$ : C, 54.79; H, 5.17; N, 13.98. Found: $\mathrm{C}, 54.47 ; \mathrm{H}, 4.89 ; \mathrm{N}, 13.49$. IR $\left(\mathrm{C}_{6} \mathrm{D}_{6}\right)$ : $2128 \mathrm{~cm}^{-1} .{ }^{1} \mathrm{H}$ NMR (400 MHz, $\mathrm{C}_{6} \mathrm{D}_{6}, 2{ }^{\circ} \mathrm{C}$ ): $\delta 7.43(\mathrm{~d}, J=1.6 \mathrm{~Hz}, 2 \mathrm{H}, 4 / 5$-imidazolylidene $H$ ), $6.76(\mathrm{~s}, 2 \mathrm{H}, \operatorname{Ar} H), 6.72(\mathrm{~s}, 2 \mathrm{H}, \operatorname{Ar} H), 6.32$ (d, 2H, 4/5-imidazolylidene H), 6.32 (s, 1H, p-pyr H), 2.85 (s, 6H, Ar- $\left.\mathrm{CH}_{3}\right), 2.15$ (s, 6H, Ar- $\left.\mathrm{CH}_{3}\right), 2.04$ (s, 6H, m-pyr- $\left.\left(\mathrm{CH}_{3}\right)_{2}\right), 1.82$ (s, 6H, Ar- $\left.\mathrm{CH}_{3}\right),-1.28$ (s, 3H, Fe-C $\left.H_{3}\right) .{ }^{13} \mathrm{C}\left\{{ }^{1} \mathrm{H}\right\}$ NMR (126 MHz, $\mathrm{C}_{6} \mathrm{D}_{6}, 25 \stackrel{\circ}{\circ} \mathrm{C}$ ): $\delta 225.2$ (2-imidazolylidene $\mathrm{C}$ ), 151.7 (opyr C), 141.4 (p-pyr C), $138.6(\operatorname{Ar} C), 138.0(\operatorname{Ar} C), 136.6(\operatorname{Ar} C), 135.7(\operatorname{Ar} C), 130.1(3-\operatorname{Ar} C)$ 128.9 (3-Ar C), 124.0 (4/5-imidazolylidene C), 118.1 (4/5-imidazolylidene C), 113.3 (m-pyr C), 21.6 $\left(\mathrm{Ar}-\mathrm{CH}_{3}\right), 21.0\left(\mathrm{Ar}-\mathrm{CH}_{3}\right), 18.5\left(\mathrm{~m}-\mathrm{pyr}-\left(\mathrm{CH}_{3}\right)_{2}\right), 18.1\left(\mathrm{Ar}-\mathrm{CH}_{3}\right)-5.5\left(\mathrm{Fe}-\mathrm{CH}_{3}\right)$.
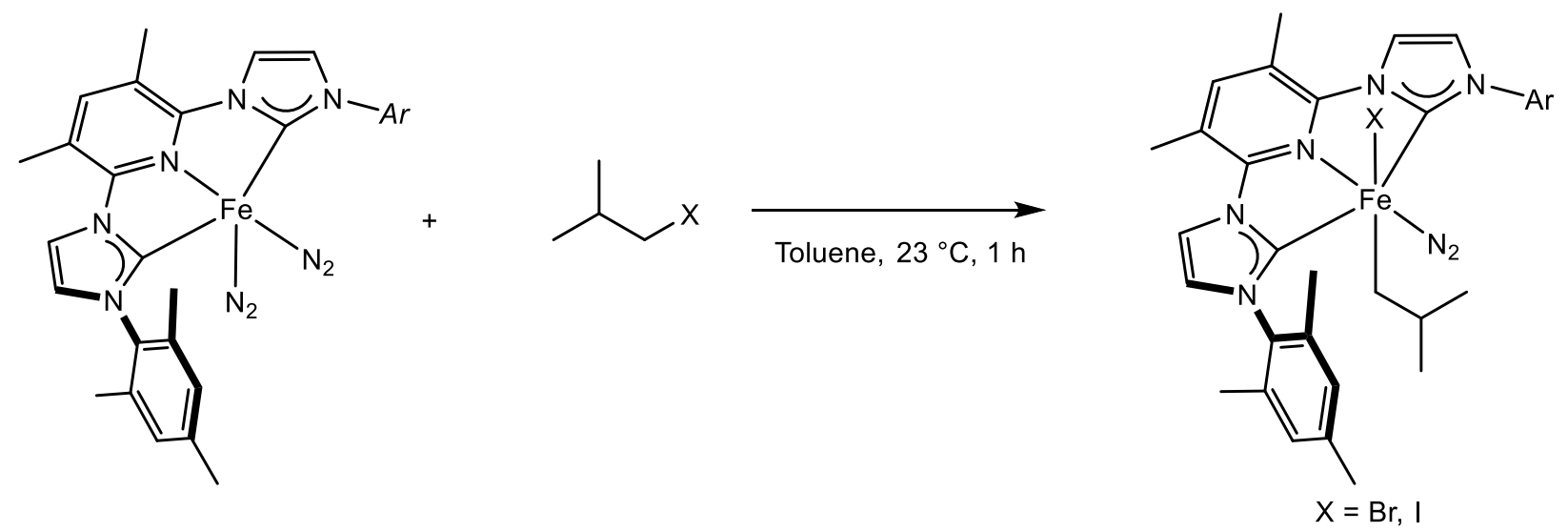

Preparation of (3,5-Me $\left.\mathrm{Me}^{-\mathrm{Mes}} \mathrm{CNC}\right) \mathrm{Fe}\left(\mathrm{CH}_{2} \mathrm{CHMe}_{2}\right)\left(\mathrm{N}_{2}\right)(\mathrm{I})$. A Schlenk tube was charged with 3,5$\mathrm{Me}_{2}{ }^{-\operatorname{Mes}} \mathrm{CNCFe}\left(\mathrm{N}_{2}\right)_{2}(52 \mathrm{mg}, 0.089 \mathrm{mmol}, 1.0$ equiv.), toluene (ca. $5 \mathrm{~mL}$ ), and a Teflon-coated stir bar. On the vacuum line, the Schlenk tube was degassed and isobutyl iodide $(0.097 \mathrm{mmol}, 1.1$ equiv.) was added via calibrated gas bulb at $-196{ }^{\circ} \mathrm{C}$. The solution was then thawed and allowed to stir at room temperature. Upon warming, the solution turned from brown to green. After stirring $1 \mathrm{~h}$, the Schlenk tube was brought into the glovebox. Pentane $(15 \mathrm{~mL})$ was added, resulting in precipitation of a green solid. The precipitate was isolated via filtration and washed with $3 \times 3 \mathrm{~mL}$ 
pentane to yield $46 \mathrm{mg}$ (69\% yield) of $\left(3,5-\mathrm{Me}_{2}{ }^{-\mathrm{Mes}} \mathrm{CNC}\right) \mathrm{Fe}\left(\mathrm{CH}_{2} \mathrm{CHMe}_{2}\right)\left(\mathrm{N}_{2}\right)(\mathrm{I})$ as a green solid. Anal Calcd for $\mathrm{C}_{35} \mathrm{H}_{42} \mathrm{FelN}_{7}$ : C, 56.54; $\mathrm{H}, 5.69 ; \mathrm{N}, 13.19$. Found: $\mathrm{C}, 56.36 ; \mathrm{H}, 5.48 ; \mathrm{N}, 12.85$. IR $\left(\mathrm{C}_{6} \mathrm{D}_{6}\right): 2130 \mathrm{~cm}^{-1} .{ }^{1} \mathrm{H}$ NMR $\left(400 \mathrm{MHz}, \mathrm{C}_{6} \mathrm{D}_{6}, 25^{\circ} \mathrm{C}\right): \delta 7.53(2 \mathrm{H}, 4 / 5$-imidazolylidene $H), 6.80(\mathrm{~s}$, 2H, Ar H), 6.67 (s, 2H, Ar H), 6.42 (s, 1H, p-pyr H), 6.33 (d, 2H, 4/5-imidazolylidene $H$ ), 2.93 (s, 6H, Ar- $\left.\mathrm{CH}_{3}\right), 2.17$ (s, 6H, Ar- $\left.\mathrm{CH}_{3}\right), 2.03$ (s, 6H, m-pyr-( $\left.\left.\mathrm{CH}_{3}\right)_{2}\right), 1.94$ (s, 6H, Ar-CH$)_{3}, 0.43$ (d, 4.8 $\left.\mathrm{Hz}, 6 \mathrm{H}, \mathrm{CH}_{2} \mathrm{CHMe}_{2}\right), 0.32$ (m, $1 \mathrm{H}, \mathrm{CH}_{2} \mathrm{CHMe}$ ), 0.09 (d, $\left.4.5 \mathrm{~Hz}, 2 \mathrm{H}, \mathrm{CH}_{2} \mathrm{CHMe}_{2}\right) .{ }^{13} \mathrm{C}\left\{{ }^{1} \mathrm{H}\right\} \mathrm{NMR}$ (126 MHz, $\mathrm{C}_{6} \mathrm{D}_{6}, 25 \stackrel{\circ}{\circ}$ ) : $\delta 223.0$ (2-imidazolylidene $\left.C\right), 152.3$ (o-pyr C), 141.0 (p-pyr C), 138.9 (Ar C), $138.5(\operatorname{Ar} C), 136.3(\operatorname{Ar} C), 135.2(\operatorname{Ar} C), 130.1(3-\operatorname{Ar} C), 128.9(3-\operatorname{Ar} C), 124.5$ (4/5imidazolylidene $C$ ), 117.7 (4/5-imidazolylidene $C$ ), 113.2 (m-pyr $C$ ), $30.9\left(\mathrm{Fe}^{-} \mathrm{CH}_{2} \mathrm{CHMe}_{2}\right), 26.6$ (Fe-CH $\left.\mathrm{CHMe}_{2}\right), 22.3\left(\mathrm{Ar}-\mathrm{CH}_{3}\right), 21.0\left(\mathrm{Ar}-\mathrm{CH}_{3}\right), 19.2\left(\mathrm{Fe}-\mathrm{CH}_{2} \mathrm{CHMe}_{2}\right), 19.0\left(\mathrm{~m}-\mathrm{pyr}-\left(\mathrm{CH}_{3}\right)_{2}\right), 18.4$ $\left(\mathrm{Ar}-\mathrm{CH}_{3}\right)$.

Preparation of (3,5-Me $\left.2^{-}{ }^{-\mathrm{Mes}} \mathrm{CNC}\right) \mathrm{Fe}\left(\mathrm{CH}_{2} \mathrm{CHMe}_{2}\right)\left(\mathrm{N}_{2}\right)(\mathrm{Br})$. The compound (3,5- $\mathrm{Me}_{2^{-}}$ $\left.{ }^{\mathrm{Mes}} \mathrm{CNC}\right) \mathrm{Fe}\left(\mathrm{CH}_{2} \mathrm{CHMe}_{2}\right)\left(\mathrm{N}_{2}\right)(\mathrm{Br})$ was prepared in an analogous fashion to $\left(3,5-\mathrm{Me}_{2}-\right.$ $\left.{ }^{\mathrm{Mes}} \mathrm{CNC}\right) \mathrm{Fe}\left(\mathrm{CH}_{2} \mathrm{CHMe}_{2}\right)\left(\mathrm{N}_{2}\right)(\mathrm{I})$ using (3,5-- $\left.\mathrm{Me}_{2}{ }^{-{ }^{M e s}} \mathrm{CNC}\right) \mathrm{Fe}\left(\mathrm{N}_{2}\right)_{2}(30 \mathrm{mg}, 0.051 \mathrm{mmol}, 1$ equiv.) and isobutyl bromide $(0.056 \mathrm{mmol}, 1.1$ equiv.). This procedure yielded $34 \mathrm{mg}(98 \%)$ of $(3,5-$ $\left.\mathrm{Me}_{2}{ }^{-{ }^{M e s}} \mathrm{CNC}\right) \mathrm{Fe}\left(\mathrm{CH}_{2} \mathrm{CHMe}_{2}\right)(\mathrm{Br})\left(\mathrm{N}_{2}\right)$ as a green solid. ${ }^{1} \mathrm{H} \mathrm{NMR}\left(400 \mathrm{MHz}, \mathrm{C}_{6} \mathrm{D}_{6}, 25^{\circ} \mathrm{C}\right): \delta 7.53$ (2H, 4/5-imidazolylidene $H$ ), 6.80 (s, 2H, $\operatorname{Ar} H$ ), 6.67 (s, 2H, $\operatorname{Ar} H$ ), 6.42 (s, 1H, p-pyr $H$ ), 6.33 (d, 2H, 4/5-imidazolylidene H), 2.93 (s, 6H, Ar- $\mathrm{CH}_{3}$ ), 2.17 (s, 6H, $\mathrm{Ar}-\mathrm{CH}_{3}$ ), 2.03 (s, 6H, m-pyr$\left.\left(\mathrm{CH}_{3}\right)_{2}\right), 1.94\left(\mathrm{~s}, 6 \mathrm{H}, \mathrm{Ar}-\mathrm{CH}_{3}\right), 0.43\left(\mathrm{~d}, 4.8 \mathrm{~Hz}, 6 \mathrm{H}, \mathrm{CH}_{2} \mathrm{CHMe}\right.$ ), $0.32\left(\mathrm{~m}, 1 \mathrm{H}, \mathrm{CH}_{2} \mathrm{CHMe}\right.$ ), 0.09 (d, $4.5 \mathrm{~Hz}, 2 \mathrm{H}, \mathrm{CH}_{2} \mathrm{CHMe}_{2}$ ). ${ }^{13} \mathrm{C}-\mathrm{NMR}$ data were not collected due to the instability of the complex in solution. 


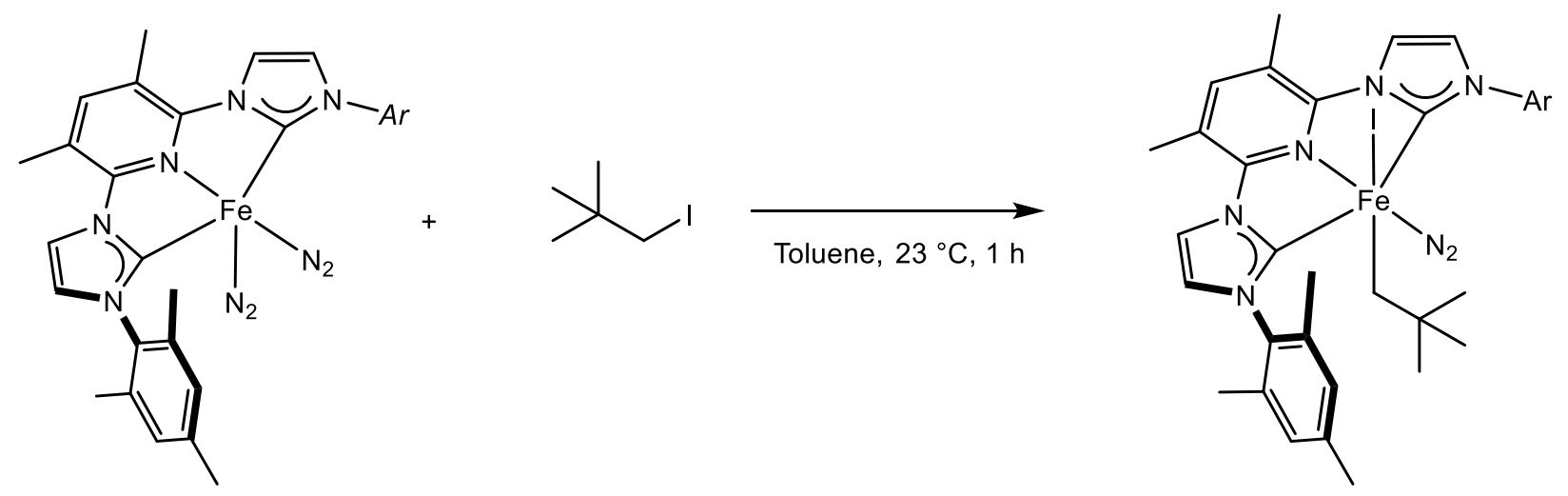

Preparation of (3,5-Me $\left.{ }^{-}{ }^{-\mathrm{Mes}} \mathrm{CNC}\right) \mathrm{Fe}\left(\mathrm{CH}_{2} \mathrm{CMe}_{3}\right)\left(\mathrm{N}_{2}\right)(\mathrm{I})$. A Schlenk tube was charged with 3,5$\mathrm{Me}_{2}{ }^{-} \operatorname{Mes} \mathrm{CNCFe}\left(\mathrm{N}_{2}\right)_{2}(100 \mathrm{mg}, 0.17 \mathrm{mmol}, 1.0$ equiv.), toluene (ca. $5 \mathrm{~mL})$, and a Teflon-coated stir bar. On the vacuum line, the Schlenk tube was degassed and neopentyl iodide $(0.19 \mathrm{mmol}$, 1.1 equiv.) was added via calibrated gas bulb at $-196^{\circ} \mathrm{C}$. The solution was then thawed and allowed to stir at room temperature. Upon warming, the solution turned from brown to green. After stirring $1 \mathrm{~h}$, the Schlenk tube was brought into the glovebox. Pentane $(15 \mathrm{~mL})$ was added, resulting in precipitation of a green solid. The precipitate was isolated via filtration and washed with $3 \times 3 \mathrm{~mL}$ pentane to yield $93 \mathrm{mg}$ (75\% yield) of $\left(3,5-\mathrm{Me}_{2}{ }^{-\mathrm{Mes}} \mathrm{CNC}\right) \mathrm{Fe}\left(\mathrm{CH}_{2} \mathrm{CMe}_{3}\right)\left(\mathrm{N}_{2}\right)(\mathrm{I})$ as a green solid. Anal Calcd for $\mathrm{C}_{36} \mathrm{H}_{44} \mathrm{FelN}_{7}$ : C, 57.08; H, 5.85; N, 12.94. Found: C, 56.72; H, 5.48; $\mathrm{N}, 13.16 .{ }^{1} \mathrm{H}$ NMR $\left(400 \mathrm{MHz}, \mathrm{C}_{6} \mathrm{D}_{6}, 25^{\circ} \mathrm{C}\right): \delta 7.55$ (d, $2.0 \mathrm{~Hz}, 2 \mathrm{H}, 4 / 5$-imidazolylidene $H$ ), 6.78 (s, 2H, Ar H), 6.67 (s, 2H, Ar H), 6.34 (m, 3H, overlap p-pyr $H$ and 4/5-imidazolylidene $H$ ), 2.91 (s, 6H, Ar- $\left.\mathrm{CH}_{3}\right), 2.30$ (s, 6H, Ar- $\left.\mathrm{CH}_{3}\right), 2.03$ (s, 6H, m-pyr- $\left.\left(\mathrm{CH}_{3}\right)_{2}\right), 1.93$ (s, 6H, Ar-CH$\left.)_{3}\right), 0.40$ (s, 9H, $\left.\mathrm{CH}_{2} \mathrm{CMe}_{3}\right), 0.32\left(\mathrm{~s}, 2 \mathrm{H}, \mathrm{CH}_{2} \mathrm{CMe}_{3}\right) .{ }^{13} \mathrm{C}\left\{{ }^{1} \mathrm{H}\right\} \mathrm{NMR}\left(126 \mathrm{MHz}, \mathrm{C}_{6} \mathrm{D}_{6}, 25{ }^{\circ} \mathrm{C}\right): \delta 223.5$ (2imidazolylidene C), 153.3 (o-pyr C), 141.0 (p-pyr C), $139.3(\operatorname{Ar} C), 138.5(\operatorname{Ar} C), 136.4(\operatorname{Ar} C)$, $134.9(\operatorname{Ar} C), 130.1(3-\operatorname{Ar} C), 129.0$ (3-Ar C), 124.6 (4/5-imidazolylidene C), 117.6 (4/5imidazolylidene $\mathrm{C}$ ), 113.2 (m-pyr $\mathrm{C}$ ), $36.1\left(\mathrm{Fe}-\mathrm{CH}_{2} \mathrm{CMe}_{3}\right), 32.6\left(\mathrm{Fe}-\mathrm{CH}_{2} \mathrm{CMe}_{3}\right), 23.9(\mathrm{Fe}-$ $\left.\mathrm{CH}_{2} \mathrm{CMe}_{3}\right), 22.6\left(\mathrm{Ar}-\mathrm{CH}_{3}\right), 20.9\left(\mathrm{Ar}-\mathrm{CH}_{3}\right), 19.5\left(\mathrm{~m}-\mathrm{pyr}-\left(\mathrm{CH}_{3}\right)_{2}\right), 18.3\left(\mathrm{Ar}-\mathrm{CH}_{3}\right)$. 

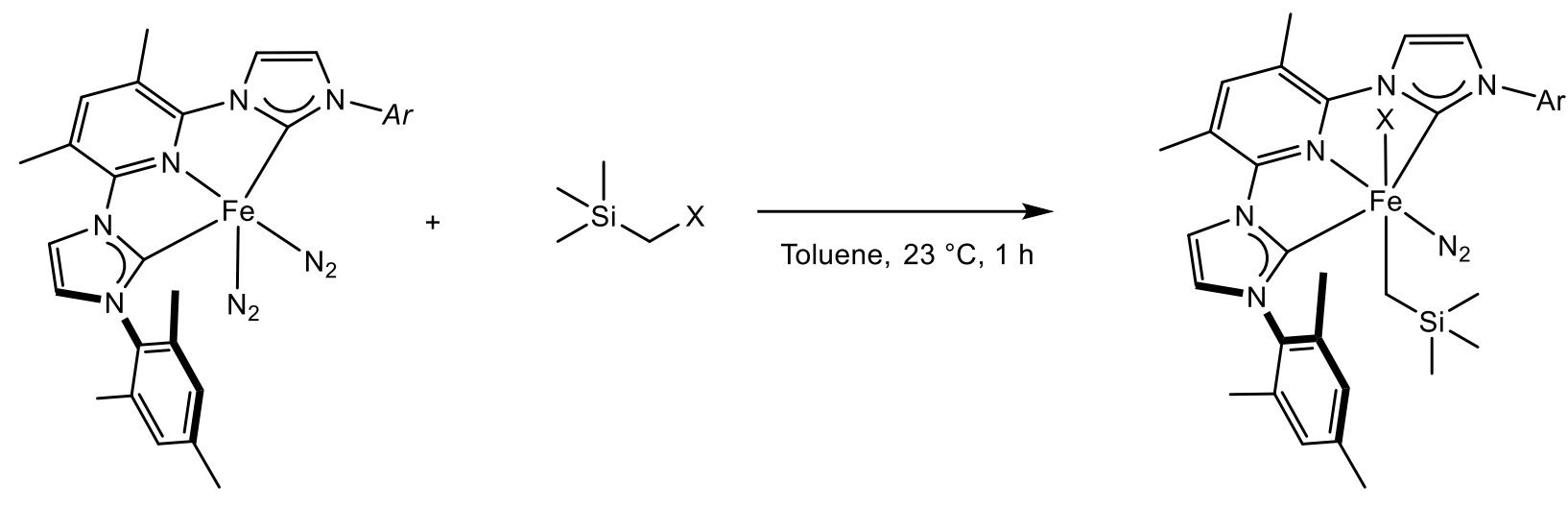

$\mathrm{X}=\mathrm{Cl}, \mathrm{Br}, \mathrm{I}$

Preparation of (3,5- $\left.\mathrm{Me}_{2}{ }^{-\mathrm{Mes}} \mathrm{CNC}\right) \mathrm{Fe}\left(\mathrm{CH}_{2} \mathrm{SiMe}_{3}\right)\left(\mathrm{N}_{2}\right)(\mathrm{Br})$. A Schlenk tube was charged with 3,5$\mathrm{Me}_{2}{ }^{-\mathrm{Mes}} \mathrm{CNCFe}\left(\mathrm{N}_{2}\right)_{2}(70 \mathrm{mg}, 0.12 \mathrm{mmol}, 1.0$ equiv.), toluene (ca. $5 \mathrm{~mL}$ ), and a Teflon-coated stir bar. On the vacuum line, the Schlenk tube was degassed and (bromomethyl)trimethylsilane (0.13 mmol, 1.1 equiv.) was added via calibrated gas bulb at $-196{ }^{\circ} \mathrm{C}$. The solution was then thawed and allowed to stir at room temperature. Upon warming, the solution turned from brown to green. After stirring $1 \mathrm{~h}$, the Schlenk tube was brought into the glovebox. Pentane (15 $\mathrm{mL})$ was added, resulting in precipitation of a green solid. The precipitate was isolated via filtration and washed with $3 \times 3 \mathrm{~mL}$ pentane to yield $70 \mathrm{mg}(78 \%$ yield $)$ of $\left(3,5-\mathrm{Me}_{2}-\right.$ $\left.{ }^{\text {Mes }} \mathrm{CNC}\right) \mathrm{Fe}\left(\mathrm{CH}_{2} \mathrm{SiMe}_{3}\right)\left(\mathrm{N}_{2}\right)(\mathrm{Br})$ as a green solid. Anal Calcd for $\mathrm{C}_{35} \mathrm{H}_{44} \mathrm{BrFeN}_{7} \mathrm{Si}$ : C, 57.85; H, 6.10; N, 13.49. Found: C, 56.47; H, 5.84; N, 12.96. ${ }^{1} \mathrm{H}$ NMR $\left(400 \mathrm{MHz}, \mathrm{C}_{6} \mathrm{D}_{6}, 25\right.$ $\left.{ }^{\circ} \mathrm{C}\right): \delta 7.53$ (bs, 2H, 4/5-imidazolylidene H), 6.84 (s, 2H, Ar H), 6.63 (s, 2H, Ar H), 6.36 (s, 1H, ppyr $H$ ), 6.32 (bs, 2H, 4/5-imidazolylidene $H$ ), 2.88 (s, 6H, $\mathrm{Ar}-\mathrm{CH}_{3}$ ), 2.13 (s, 6H, Ar-CH3), 2.03 (s, 6H, m-pyr-( $\left.\left.\mathrm{CH}_{3}\right)_{2}\right), 1.95$ (s, 6H, $\left.\mathrm{Ar}-\mathrm{CH}_{3}\right),-0.48$ (s, 9H, $\mathrm{CH}_{2} \mathrm{SiMe}$ ), -1.91 (s, 2H, $\mathrm{CH}_{2} \mathrm{SiMe}_{3}$ ). ${ }^{13} \mathrm{C}\left\{{ }^{1} \mathrm{H}\right\}$ NMR (126 MHz, $\left.\mathrm{C}_{6} \mathrm{D}_{6}, 25^{\circ} \mathrm{C}\right): \delta 222.1$ (2-imidazolylidene $\left.C\right), 153.3$ (o-pyr $\left.\mathrm{C}\right), 141.5$ (ppyr C), $139.0(\operatorname{Ar} C), 138.6(\operatorname{Ar} C), 136.4(\operatorname{Ar} C), 134.7(\operatorname{Ar} C), 129.9(3-\operatorname{Ar} C), 129.1(3-\operatorname{Ar} C)$, 124.2 (4/5-imidazolylidene C), 118.0 (4/5-imidazolylidene $C), 113.4$ (m-pyr $C), 21.0\left(\mathrm{Ar}-\mathrm{CH}_{3}\right)$, $20.7\left(\mathrm{Ar}-\mathrm{CH}_{3}\right), 18.7\left(\mathrm{~m}-\mathrm{pyr}-\left(\mathrm{CH}_{3}\right)_{2}\right), 18.3\left(\mathrm{Ar}-\mathrm{CH}_{3}\right), 2.1\left(\mathrm{Fe}-\mathrm{CH}_{2} \mathrm{SiMe}_{3}\right)$. 
Preparation of (3,5-Me $\left.{ }_{2}{ }^{-\mathrm{Mes}} \mathrm{CNC}\right) \mathrm{Fe}\left(\mathrm{CH}_{2} \mathrm{SiMe}_{3}\right)\left(\mathrm{N}_{2}\right)(\mathrm{I})$. The compound (3,5-Me ${ }_{2-}^{-}$ $\left.{ }^{\mathrm{Mes}} \mathrm{CNC}\right) \mathrm{Fe}\left(\mathrm{CH}_{2} \mathrm{SiMe}_{3}\right)\left(\mathrm{N}_{2}\right)$ (I) was prepared in an analogous fashion to $\left(3,5-\mathrm{Me}_{2^{-}}\right.$ $\left.{ }^{\mathrm{Mes}} \mathrm{CNC}\right) \mathrm{Fe}\left(\mathrm{CH}_{2} \mathrm{SiMe}_{3}\right)\left(\mathrm{N}_{2}\right)(\mathrm{I})$ using $3,5-\mathrm{Me}_{2}{ }^{-\mathrm{Mes}} \mathrm{CNCFe}\left(\mathrm{N}_{2}\right)_{2}(100 \mathrm{mg}, 0.17 \mathrm{mmol}, 1.0$ equiv. and (iodomethyl)trimethylsilane ( $0.19 \mathrm{mmol}, 1.1$ equiv.). This procedure yielded $114 \mathrm{mg}(84 \%$ yield) of $\left(3,5-\mathrm{Me}_{2}{ }^{-{ }^{M e s}} \mathrm{CNC}\right) \mathrm{Fe}\left(\mathrm{CH}_{2} \mathrm{SiMe}_{3}\right)\left(\mathrm{N}_{2}\right)(\mathrm{I})$ as a green solid. ${ }^{1} \mathrm{H} \mathrm{NMR}\left(400 \mathrm{MHz}, \mathrm{C}_{6} \mathrm{D}_{6}, 25\right.$ ${ }^{\circ} \mathrm{C}$ ): $\delta 7.54$ (d, $1.8 \mathrm{~Hz}, 2 \mathrm{H}, 4 / 5$-imidazolylidene H), 6.81 (s, 2H, Ar H), 6.60 (s, 2H, Ar H), 6.38 (s, 1H, p-pyr H), $6.32(\mathrm{~d}, 1.8 \mathrm{~Hz}, 2 \mathrm{H}, 4 / 5$-imidazolylidene H), 2.92 (s, 6H, Ar-CH$), 2.11$ (s, 6H, Ar$\left.\mathrm{CH}_{3}\right), 2.02\left(\mathrm{~s}, 6 \mathrm{H}, \mathrm{m}-\mathrm{pyr}-\left(\mathrm{CH}_{3}\right)_{2}\right), 1.96\left(\mathrm{~s}, 6 \mathrm{H}, \mathrm{Ar}-\mathrm{CH}_{3}\right),-0.48\left(\mathrm{~s}, 9 \mathrm{H}, \mathrm{CH}_{2} \mathrm{SiMe}\right),-1.76(\mathrm{~s}, 2 \mathrm{H}$, $\left.\mathrm{CH}_{2} \mathrm{SiMe}_{3}\right) .{ }^{13} \mathrm{C}\left\{{ }^{1} \mathrm{H}\right\} \mathrm{NMR}\left(126 \mathrm{MHz}, \mathrm{C}_{6} \mathrm{D}_{6}, 25^{\circ} \mathrm{C}\right.$ ): $\delta 223.0$ (2-imidazolylidene $\mathrm{C}$ ), 153.0 (o-pyr C), $141.4(\mathrm{p}-\mathrm{pyr} C), 139.0(\operatorname{Ar} C), 138.6(\operatorname{Ar} C), 136.3(\operatorname{Ar} C), 134.9(\operatorname{Ar} C), 130.0(3-\operatorname{Ar} C), 129.2$ (3-Ar C), 124.5 (4/5-imidazolylidene C), 117.9 (4/5-imidazolylidene $C$ ), 113.3 (m-pyr C), 22.1 (Ar- $\left.\mathrm{CH}_{3}\right), 21.0\left(\mathrm{Ar}-\mathrm{CH}_{3}\right), 18.9\left(\mathrm{~m}-\mathrm{pyr}-\left(\mathrm{CH}_{3}\right)_{2}\right), 18.2\left(\mathrm{Ar}-\mathrm{CH}_{3}\right), 2.2\left(\mathrm{Fe}-\mathrm{CH}_{2} \mathrm{SiMe}\right)_{3}$.

Preparation of (3,5-Me $\left.{ }_{2}{ }^{-}{ }^{\mathrm{Mes}} \mathrm{CNC}\right) \mathrm{Fe}\left(\mathrm{CH}_{2} \mathrm{SiMe}_{3}\right)\left(\mathrm{N}_{2}\right)(\mathrm{Cl})$. The compound $\left(3,5-\mathrm{Me}_{2-}\right.$ $\left.{ }^{\mathrm{Mes}} \mathrm{CNC}\right) \mathrm{Fe}\left(\mathrm{CH}_{2} \mathrm{SiMe}_{3}\right)\left(\mathrm{N}_{2}\right)(\mathrm{Cl})$ was prepared in an analogous fashion to $\left(3,5-\mathrm{Me}_{2}-\right.$ $\left.{ }^{\mathrm{Mes}} \mathrm{CNC}\right) \mathrm{Fe}\left(\mathrm{CH}_{2} \mathrm{SiMe}_{3}\right)\left(\mathrm{N}_{2}\right)(\mathrm{Br})$ using $3,5-\mathrm{Me}_{2}{ }^{-\mathrm{Mes}} \mathrm{CNCFe}\left(\mathrm{N}_{2}\right)_{2}(50 \mathrm{mg}, 0.085 \mathrm{mmol}, 1.0$ equiv. $)$ and (chloromethyl)trimethylsilane $(0.10 \mathrm{mmol}, 1.1$ equiv.). This procedure yielded $30 \mathrm{mg}$ (50\% yield) of $\left(3,5-\mathrm{Me}_{2}{ }^{-{ }^{-}{ }^{-s}} \mathrm{CNC}\right) \mathrm{Fe}\left(\mathrm{CH}_{2} \mathrm{SiMe}_{3}\right)\left(\mathrm{N}_{2}\right)(\mathrm{Cl})$ as a green solid. ${ }^{1} \mathrm{H} \mathrm{NMR}\left(400 \mathrm{MHz}, \mathrm{C}_{6} \mathrm{D}_{6}, 25\right.$ ${ }^{\circ} \mathrm{C}$ ): $\delta 7.51$ (bs, 2H, 4/5-imidazolylidene H), 6.84 (s, 2H, Ar H), 6.65 (s, 2H, Ar H), 6.38 (s, 1H, ppyr H), 6.34 (bs, 2H, 4/5-imidazolylidene H), 2.84 (s, 6H, Ar- $\mathrm{CH}_{3}$ ), 2.14 (s, 6H, Ar-CH$)_{3}$ ), 2.04 (s, $\left.6 \mathrm{H}, \mathrm{m}-\mathrm{pyr}-\left(\mathrm{CH}_{3}\right)_{2}\right), 1.93\left(\mathrm{~s}, 6 \mathrm{H}, \mathrm{Ar}-\mathrm{CH}_{3}\right),-0.49$ (s, 9H, $\left.\mathrm{CH}_{2} \mathrm{SiMe}_{3}\right),-1.89$ (bs, $\left.2 \mathrm{H}, \mathrm{CH}_{2} \mathrm{SiMe}_{3}\right) .{ }^{13} \mathrm{C}-$ NMR data were not collected due to the instability of the complex in solution. 

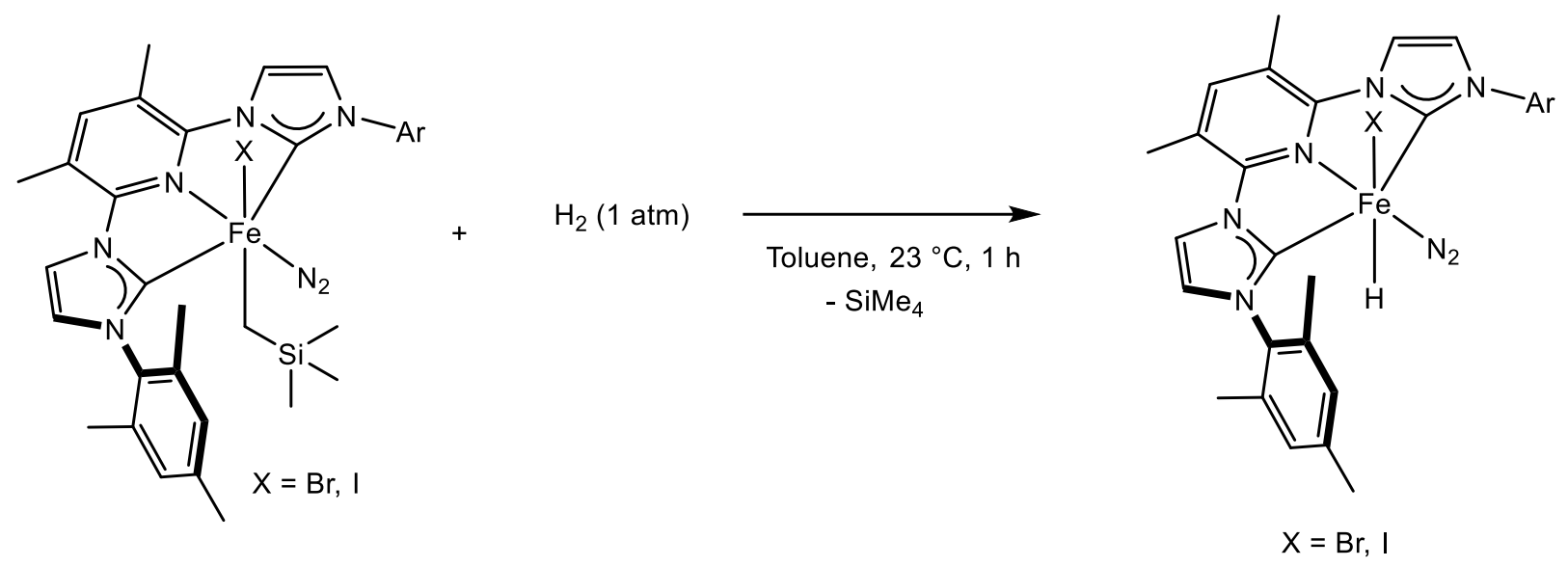

Preparation of (3,5-Me $\left.2^{-}{ }^{-\mathrm{Mes}} \mathrm{CNC}\right) \mathrm{Fe}(\mathrm{H})\left(\mathrm{N}_{2}\right)(\mathrm{Br})$. A Schlenk tube was charged with (3,5- $\mathrm{Me}_{2^{-}}$ ${ }^{\text {Mes }} \mathrm{CNCFe}\left(\mathrm{CH}_{2} \mathrm{CHMe}_{2}\right)\left(\mathrm{N}_{2}\right)(\mathrm{Br})(50 \mathrm{mg}, 0.085 \mathrm{mmol}, 1.0$ equiv.), toluene (ca. $2 \mathrm{~mL})$, and a Teflon-coated stir bar. On the vacuum line, the Schlenk tube was degassed and hydrogen (1 atm.) was added. The solution was then thawed and allowed to stir at room temperature. Upon warming, the solution turned from green to red-brown. After stirring $1 \mathrm{~h}$, the Schlenk tube was brought into the glovebox. Pentane $(10 \mathrm{~mL})$ was added, resulting in precipitation of a brown solid. The precipitate was isolated via filtration and washed with $3 \times 3 \mathrm{~mL}$ pentane to yield 28 mg (52\% yield) of $\left(3,5-\mathrm{Me}_{2}{ }^{-\mathrm{Mes}} \mathrm{CNC}\right) \mathrm{Fe}(\mathrm{H})\left(\mathrm{N}_{2}\right)(\mathrm{Br})$ as a red solid. Anal Calcd for $\mathrm{C}_{31} \mathrm{H}_{34} \mathrm{BrFeN}_{7}$ : C, 58.14; H, 5.35; N, 15.31. Found: C, 58.19; H, 4.90; N, 14.87. IR $\left(\mathrm{C}_{6} \mathrm{D}_{6}\right): 2134 \mathrm{~cm}^{-1} .{ }^{1} \mathrm{H}$ NMR (400 MHz, $\left.\mathrm{C}_{6} \mathrm{D}_{6}, 25^{\circ} \mathrm{C}\right): \delta 7.34(\mathrm{~d}, 2.2 \mathrm{~Hz}, 2 \mathrm{H}, 4 / 5$-imidazolylidene $H$ ), 6.77 (s, 2H, Ar H), 6.67 (s, 2H, Ar H), 6.36 (d, $2.2 \mathrm{~Hz}, 2 \mathrm{H}, 4 / 5$-imidazolylidene H), 6.23 (s, 1H, p-pyr H), 2.78 (s, 6H, Ar$\left.\mathrm{CH}_{3}\right), 2.25\left(\mathrm{~s}, 6 \mathrm{H}, \mathrm{Ar}-\mathrm{CH}_{3}\right), 2.03\left(\mathrm{~s}, 6 \mathrm{H}, \mathrm{m}-\mathrm{pyr}-\left(\mathrm{CH}_{3}\right)_{2}\right), 1.75\left(\mathrm{~s}, 6 \mathrm{H}, \mathrm{Ar}-\mathrm{CH}_{3}\right),-21.71(\mathrm{~s}, 1 \mathrm{H}, \mathrm{Fe}-$ H). ${ }^{13} \mathrm{C}\left\{{ }^{1} \mathrm{H}\right\}$ NMR (126 MHz, $\mathrm{C}_{6} \mathrm{D}_{6}, 25^{\circ} \mathrm{C}$ ): $\delta 220.9$ (2-imidazolylidene C), 152.1 (o-pyr C), 140.8 (p-pyr C), $138.7(\operatorname{Ar} C), 137.9(\operatorname{Ar} C), 136.7(\operatorname{Ar} C), 135.9(\operatorname{Ar} C), 129.9(3-\operatorname{Ar} C), 128.8(3-\operatorname{Ar} C)$, 123.3 (4/5-imidazolylidene C), 117.5 (4/5-imidazolylidene $C$ ), 112.6 (m-pyr C), $21.1\left(\mathrm{Ar}-\mathrm{CH}_{3}\right)$, $19.8\left(\mathrm{Ar}-\mathrm{CH}_{3}\right), 18.4\left(\mathrm{~m}-\mathrm{pyr}-\left(\mathrm{CH}_{3}\right)_{2}\right), 18.0\left(\mathrm{Ar}-\mathrm{CH}_{3}\right)$.

Preparation of (3,5-Me $\left.2^{-{ }^{-}}{ }^{-s} \mathrm{CNC}\right) \mathrm{Fe}(\mathrm{H})\left(\mathrm{N}_{2}\right)(\mathrm{I})$ The compound $\left(3,5-\mathrm{Me}_{2}{ }^{-\mathrm{Mes}} \mathrm{CNC}\right) \mathrm{Fe}(\mathrm{H})\left(\mathrm{N}_{2}\right)(\mathrm{I})$ was prepared in an analogous fashion to $\left(3,5-\mathrm{Me}_{2}{ }^{-}{ }^{\mathrm{Mes}} \mathrm{CNC}\right) \mathrm{Fe}(\mathrm{H})\left(\mathrm{N}_{2}\right)(\mathrm{Br})$ using $\left(3,5-\mathrm{Me}_{2^{-}}\right.$ 
${ }^{\text {Mes }} \mathrm{CNCFe}\left(\mathrm{CH}_{2} \mathrm{CHMe}_{2}\right)\left(\mathrm{N}_{2}\right)(\mathrm{I})(57 \mathrm{mg}, 0.077 \mathrm{mmol}, 1.0$ equiv.) and hydrogen (1 atm.). This procedure yielded $36 \mathrm{mg}$ (68\% yield) of $\left(3,5-\mathrm{Me}_{2}{ }^{-\mathrm{Mes}} \mathrm{CNC}\right) \mathrm{Fe}(\mathrm{H})\left(\mathrm{N}_{2}\right)(\mathrm{I})$ as a light red-brown solid. ${ }^{1} \mathrm{H}$ NMR $\left(400 \mathrm{MHz}, \mathrm{C}_{6} \mathrm{D}_{6}, 25^{\circ} \mathrm{C}\right): \delta 7.36$ (d, $2.2 \mathrm{~Hz}, 2 \mathrm{H}, 4 / 5$-imidazolylidene $H$ ), 6.77 (s, 2H, Ar H), $6.66(\mathrm{~s}, 2 \mathrm{H}, \operatorname{Ar} H), 6.36(\mathrm{~d}, 2.2 \mathrm{~Hz}, 2 \mathrm{H}, 4 / 5$-imidazolylidene $H), 6.23(\mathrm{~s}, 1 \mathrm{H}, \mathrm{p}-\mathrm{pyr} H)$, 2.80 (s, 6H, Ar- $\mathrm{CH}_{3}$ ), 2.29 (s, 6H, Ar- $\left.\mathrm{CH}_{3}\right), 2.01$ (s, 6H, m-pyr- $\left.\left(\mathrm{CH}_{3}\right)_{2}\right), 1.77$ (s, 6H, Ar- $\mathrm{CH}_{3}$ ), $19.54(\mathrm{~s}, 1 \mathrm{H}, \mathrm{Fe}-H) \cdot{ }^{13} \mathrm{C}\left\{{ }^{1} \mathrm{H}\right\} \mathrm{NMR}\left(126 \mathrm{MHz}, \mathrm{C}_{6} \mathrm{D}_{6}, 25^{\circ} \mathrm{C}\right): \delta 221.5$ (2-imidazolylidene $\left.\mathrm{C}\right), 151.7$ (o-pyr C), 140.8 (p-pyr C), $138.7(\operatorname{Ar} C), 137.6(\operatorname{Ar} C), 136.5(\operatorname{Ar} C), 136.0(\operatorname{Ar} C), 129.9(3-\operatorname{Ar} C)$, 128.9 (3-Ar C), 123.5 (4/5-imidazolylidene C), 117.5 (4/5-imidazolylidene C), 112.6 (m-pyr C), $21.0\left(\mathrm{Ar}-\mathrm{CH}_{3}\right), 20.5\left(\mathrm{Ar}-\mathrm{CH}_{3}\right), 18.4\left(\mathrm{~m}-\mathrm{pyr}-\left(\mathrm{CH}_{3}\right)_{2}\right), 18.0\left(\mathrm{Ar}-\mathrm{CH}_{3}\right)$. The isotopologue (3,5- $\mathbf{M e}_{2}-$ $\left.{ }^{\text {Mes }} \mathrm{CNC}\right) \mathrm{Fe}(\mathrm{D})\left(\mathbf{N}_{2}\right)(\mathrm{I})$ was prepared analogously using $\mathrm{D}_{2}$ gas and characterized by a signal in the ${ }^{2} \mathrm{H}-\mathrm{NMR}$ spectrum at $-19.5 \mathrm{ppm}\left(\mathrm{C}_{6} \mathrm{H}_{6}, 25^{\circ} \mathrm{C}\right)$.
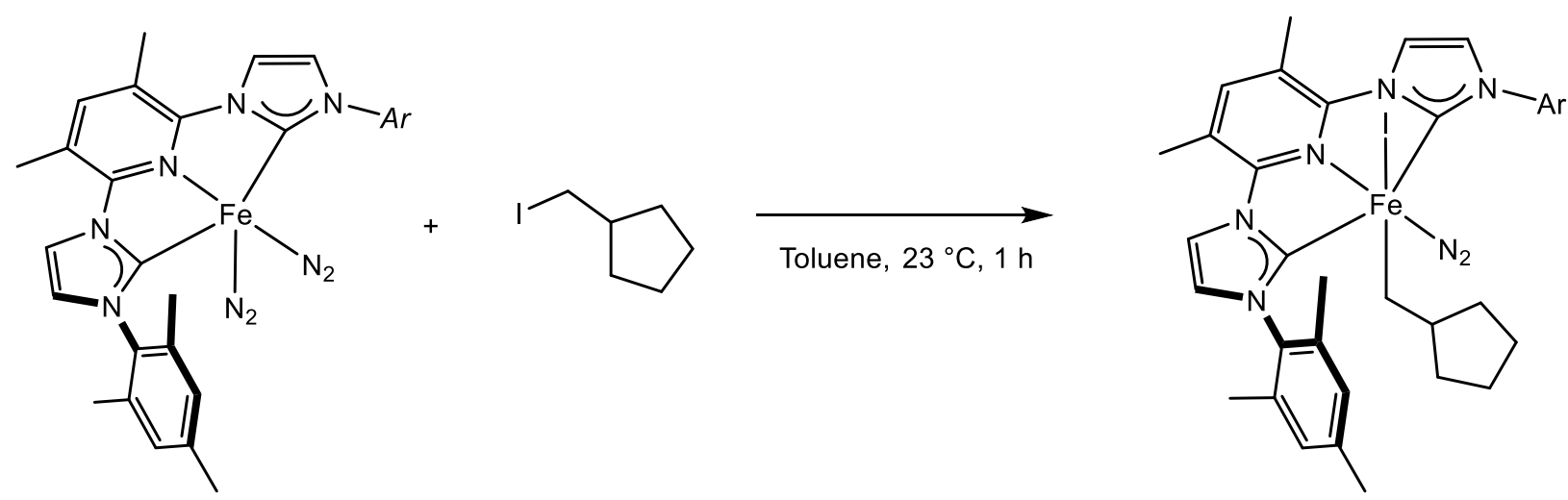

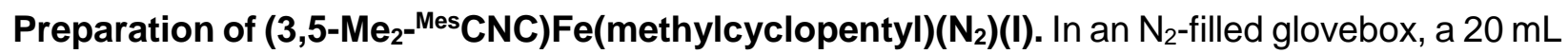
scintillation vial was charged with $3,5-\mathrm{Me}_{2}{ }^{-\mathrm{Mes}} \mathrm{CNCFe}\left(\mathrm{N}_{2}\right)_{2}(40 \mathrm{mg}, 0.068 \mathrm{mmol}, 1.0$ equiv.), toluene (ca. $5 \mathrm{~mL}$ ), and a Teflon-coated stir bar. To the stirring solution was added (iodomethyl)cyclopentane $(9.8 \mu \mathrm{L}, 0.075 \mathrm{mmol}, 1.1$ equiv.) neat via syringe. After stirring $1 \mathrm{~h}$, pentane $(15 \mathrm{~mL})$ was added, resulting in precipitation of a green solid. The precipitate was isolated via filtration and washed with $3 \times 3 \mathrm{~mL}$ pentane to yield $26 \mathrm{mg}$ (50\% yield) of $\left(3,5-\mathrm{Me}_{2}-\right.$ ${ }^{\mathrm{Mes}} \mathrm{CNC}$ ) $\mathrm{Fe}$ (methylcyclopentyl) $\left(\mathrm{N}_{2}\right)(\mathrm{I})$ as a green solid. Anal Calcd for $\mathrm{C}_{35} \mathrm{H}_{42} \mathrm{FelN} \mathrm{N}_{7}$ : C, 57.23; $\mathrm{H}$, 5.60; N, 12.98. Found: $\mathrm{C}, 56.97 ; \mathrm{H}, 6.00 ; \mathrm{N}, 13.07 .{ }^{1} \mathrm{H}$ NMR $\left(400 \mathrm{MHz}, \mathrm{C}_{6} \mathrm{D}_{6}, 25^{\circ} \mathrm{C}\right): \delta 7.51(2 \mathrm{H}$, 
4/5-imidazolylidene H), 6.81 (s, 2H, Ar H), 6.66 (s, 2H, Ar H), 6.41 (s, 1H, p-pyr H), 6.31 (d, 2H, 4/5-imidazolylidene H), 2.95 (s, 6H, Ar- $\mathrm{CH}_{3}$ ), 2.14 (s, 6H, Ar- $\mathrm{CH}_{3}$ ), 2.03 (s, 6H, m-pyr-( $\left.\mathrm{CH}_{3}\right)_{2}$ ), 1.94 (s, 6H, Ar-CH $\mathrm{CH}_{3}, 1.38$ (m, 2H, Сур-H), 1.17 (m, 2H, Сур-H), 1.00 (m, 2H, Сyp-H), 0.54 (m, 3H, Cyp-H), 0.23 (d, $\left.6.1 \mathrm{~Hz}, 2 \mathrm{H}, \mathrm{CH}_{2}(\mathrm{Cyp})\right) .{ }^{13} \mathrm{C}\left\{{ }^{1} \mathrm{H}\right\} \mathrm{NMR}\left(126 \mathrm{MHz}, \mathrm{C}_{6} \mathrm{D}_{6}, 25 \stackrel{\circ}{\circ} \mathrm{C}\right): \delta 222.8$ (2imidazolylidene C), 152.2 (o-pyr C), 140.9 (p-pyr C), 138.9 ( $\operatorname{Ar} C), 138.5(\operatorname{Ar} C), 136.3(\operatorname{Ar} C)$, 135.1 ( $\operatorname{Ar} C), 130.0$ (3-Ar C), 128.9 (3-Ar C), 124.5 (4/5-imidazolylidene C), 117.6 (4/5imidazolylidene C), 113.1 (m-pyr C), 43.0 (Сур C), 36.2 (Сур C), 26.8 (Сур C), $22.2\left(\mathrm{Ar}-\mathrm{CH}_{3}\right.$ ), $21.0\left(\mathrm{Ar}-\mathrm{CH}_{3}\right), 19.2\left(\mathrm{Fe}-\mathrm{CH}_{2}(\mathrm{cyp})\right), 18.8\left(\mathrm{~m}-\mathrm{pyr}-\left(\mathrm{CH}_{3}\right)_{2}\right), 18.3\left(\mathrm{Ar}-\mathrm{CH}_{3}\right)$.

\section{Additional Reactions and Associated NMR Data.}

\section{Stereochemical Probe Experiment}

The synthesis of the model stereochemical substrate threo-ICHDCHDC $\left(\mathrm{CH}_{3}\right)_{3}$ was adapted from the literature. ${ }^{4}$ Specifically, a modified procedure for the synthesis of neohexene- $d_{2}$ was employed.

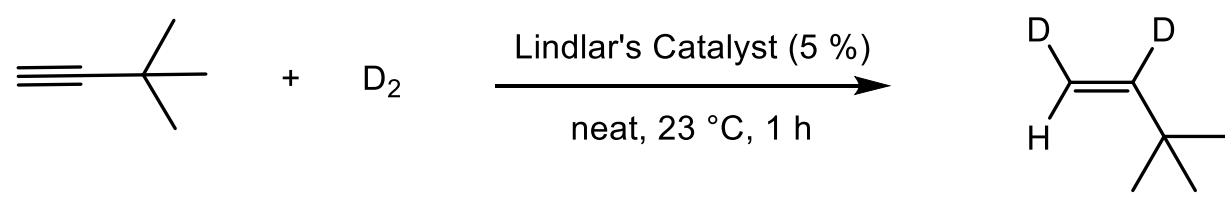

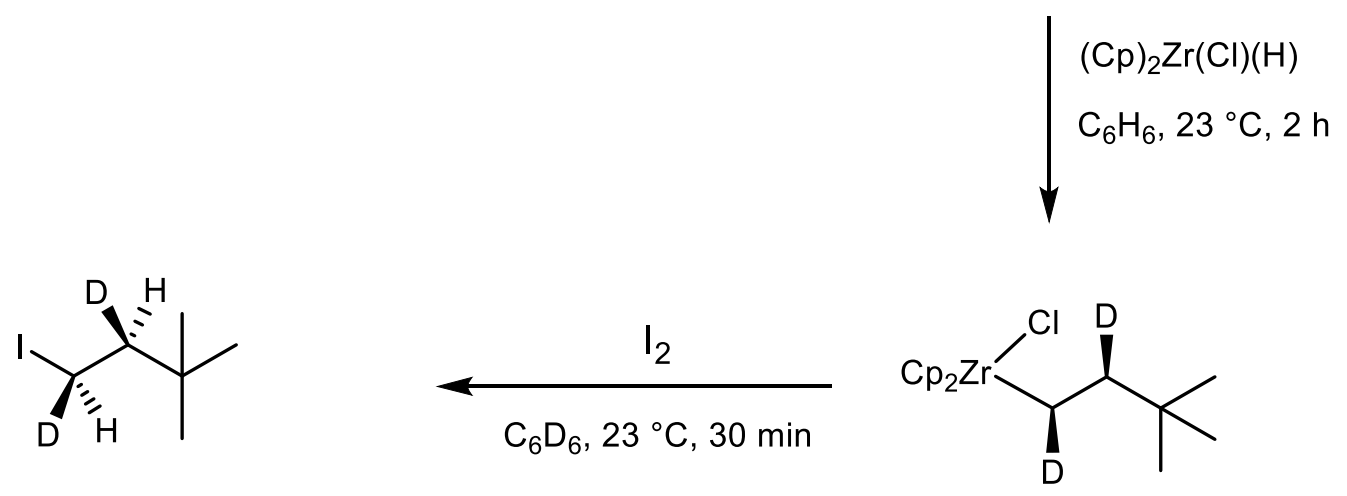

Synthesis of neohexene- $d_{2} \ln$ a $200 \mathrm{~mL}$ Schlenk tube was combined 3,3-Dimethyl-1-butyne 
(1 mL, $8.12 \mathrm{mmol}, 1$ equiv) and Lindlar's Catalyst (44 mg, $5 \%$ ). On a Schlenk line, the tube was frozen in liquid nitrogen $\left(-196^{\circ} \mathrm{C}\right)$ and the solution degassed via three freeze-pump-thaw cycles, and 4 atm $D_{2}$ was added. The solution was stirred for $1 \mathrm{~h}$, then vacuum distilled to a new Schlenk tube, giving quantitative formation of neohexene- $d_{2}$. Subsequent analysis by ${ }^{1} \mathrm{H}-\mathrm{NMR}$ and ${ }^{2} \mathrm{H}$ NMR matched the previously reported spectrum for neohexene- $d_{2 .}{ }^{4}$
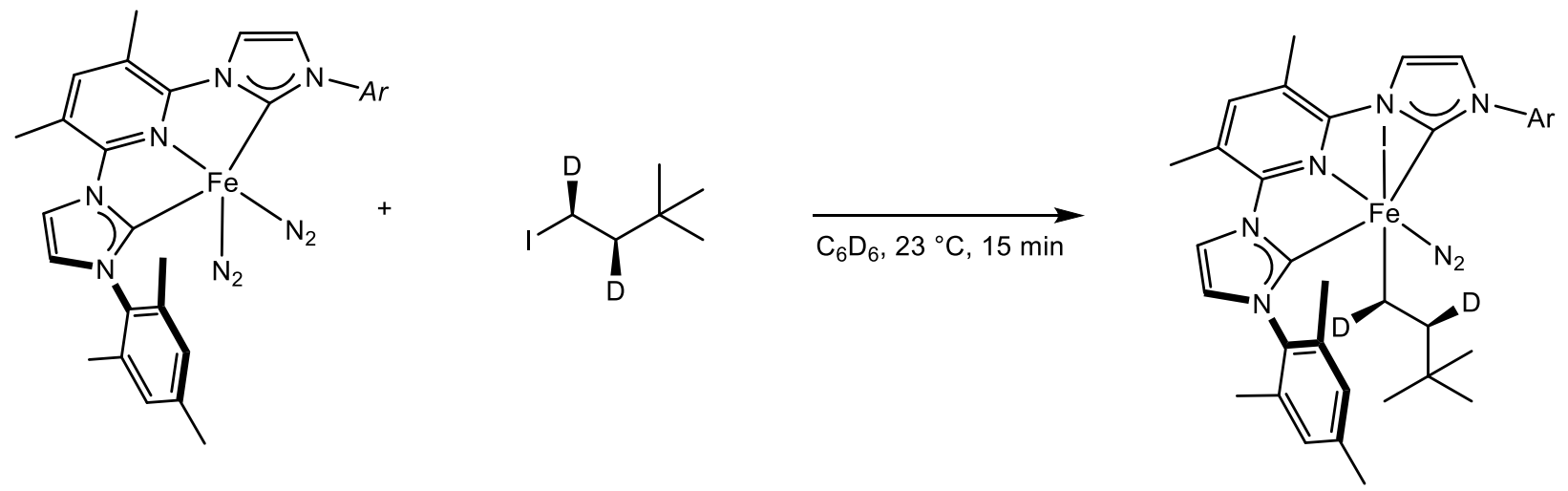

Preparation of 1-neohexyl $\left(\mathbf{N}_{2}\right)$ I In an $\mathrm{N}_{2}$-filled glovebox, a scintillation vial was charged with 15 mg of threo- $\left(\eta^{5}-\mathrm{C}_{5} \mathrm{H}_{5}\right)_{2} \mathrm{Zr}(\mathrm{Cl})\left(\mathrm{CHDCHDC}\left(\mathrm{CH}_{3}\right)_{3}\right)(0.044 \mathrm{mmol}, 1$ equiv. $)$ and $0.5 \mathrm{~mL}$ of $\mathrm{C}_{6} \mathrm{D}_{6}$. In a separate vial, $12 \mathrm{mg} \mathrm{I}_{2}$ ( 0.044 mmol, 1 equiv.) was dissolved in $0.5 \mathrm{~mL}$ of $\mathrm{C}_{6} \mathrm{D}_{6}$. The $\mathrm{I}_{2}$ solution was added dropwise to the $[\mathrm{Zr}]$ solution and stirred for 15 minutes, resulting in a color change from deep purple to light purple. The solution was then filtered through alumina, giving a colorless solution. Analysis by ${ }^{1} \mathrm{H}-\mathrm{NMR}$ was consistent with the previously reported spectroscopic data for threo-ICHDCHDC $\left(\mathrm{CH}_{3}\right)_{3}{ }^{4}$ The solution of threo-ICHDCHDC $\left(\mathrm{CH}_{3}\right)_{3}$ in $1 \mathrm{~mL} \mathrm{C}_{6} \mathrm{D}_{6}$ was added to a solution of $1-\left(\mathbf{N}_{2}\right)_{2}$ in $0.5 \mathrm{~mL} \mathrm{C} \mathrm{D}_{6}$ and stirred for 15 minutes at $23{ }^{\circ} \mathrm{C}$. An immediate color change from brown to green was observed. The resulting solution was filtered and characterized by ${ }^{1} \mathrm{H}$ and ${ }^{1} \mathrm{H}\left\{{ }^{2} \mathrm{H}\right\} \mathrm{NMR}$, and was consistent with formation of 1-neohexyl( $\left.\mathbf{N}_{2}\right) \mathbf{l}$. ${ }^{1} \mathrm{H} N M R\left(400 \mathrm{MHz}, \mathrm{C}_{6} \mathrm{D}_{6}\right.$, $25^{\circ} \mathrm{C}$ ): $\delta 7.43$ (s, 2H, 4/5-imidazolylidene $H$ ), 6.77 (s, 4H, Ar H), 6.60 (s, 1H, p-pyr H), 6.23 (d, 2H, 4/5-imidazolylidene H), 2.76 (s, 6H, Ar- $\left.\mathrm{CH}_{3}\right), 2.13$ (s, 6H, $\left.\mathrm{Ar}-\mathrm{CH}_{3}\right), 2.07$ (s, 6H, m-pyr- $\left.\left(\mathrm{CH}_{3}\right)_{2}\right)$, $1.96\left(\mathrm{~s}, 6 \mathrm{H}, \mathrm{Ar}-\mathrm{CH}_{3}\right), 0.68\left(\mathrm{~s}, 9 \mathrm{H}, \mathrm{Fe}-\mathrm{CHDCHDC}\left(\mathrm{CH}_{3}\right)_{3}\right),-0.17\left(\mathrm{~m}, 1 \mathrm{H}, \mathrm{Fe}-\mathrm{CHDCHDC}\left(\mathrm{CH}_{3}\right)_{3}\right)$, - 
$0.26\left(\mathrm{~m}, 1 \mathrm{H}, \mathrm{Fe}-\mathrm{CHDCHDC}\left(\mathrm{CH}_{3}\right)_{3}\right)$. The complex was sensitive to isolation, so further analytical data were not acquired.

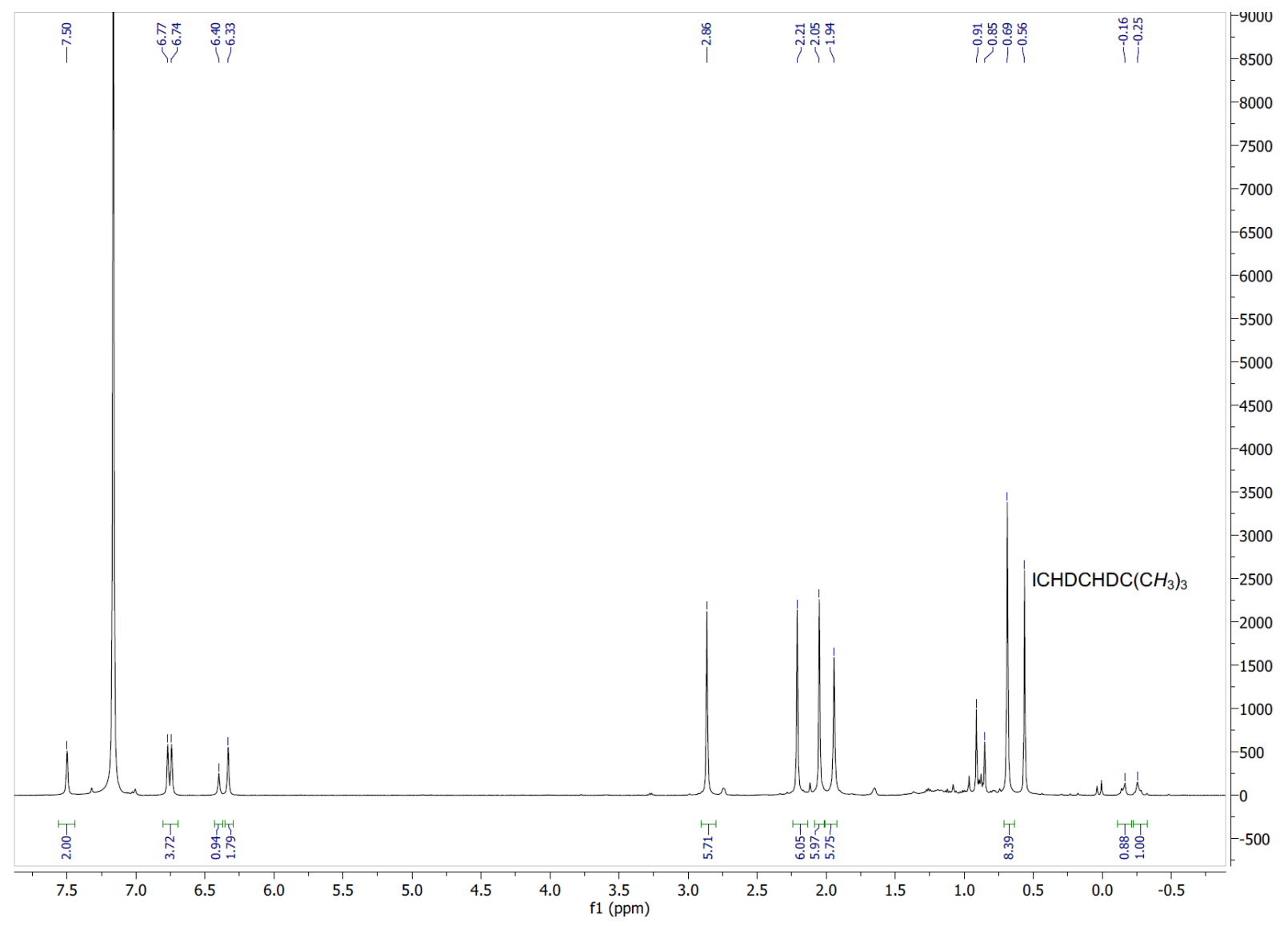

Figure S1. ${ }^{1} \mathrm{H}-\mathrm{NMR}$ spectrum of $\left(3,5-\mathrm{Me}_{2-}{ }^{\mathrm{Mes}} \mathrm{CNC}\right) \mathrm{Fe}\left(\right.$ neohexyl- $\left.d_{2}\right)\left(\mathrm{N}_{2}\right) \mathrm{I}\left(\mathbf{1}\right.$-neohexyl $\left.\left(\mathbf{N}_{2}\right) \mathrm{I}\right)$ in $\mathrm{C}_{6} \mathrm{D}_{6}$ at $25^{\circ} \mathrm{C}$.

Preparation of 1-neohexyl(CO)I. A solution of 1-neohexyl( $\left.\mathbf{N}_{\mathbf{2}}\right) \mathrm{I}$ in $\mathrm{C}_{6} \mathrm{D}_{6}$ in a J. Young NMR tube was degassed via three freeze-pump-thaw cycles, then exposed to $\mathrm{CO}(0.1 \mathrm{~atm})$. After thawing, the solution changed color from green to purple. The resulting solution was characterized by ${ }^{1} \mathrm{H}$ and ${ }^{1} \mathrm{H}\left\{{ }^{2} \mathrm{H}\right\} \mathrm{NMR}$, and was consistent with formation of 1-neohexyl(CO)I. ${ }^{1} \mathrm{H}$ NMR $(400 \mathrm{MHz}$, $\left.\mathrm{C}_{6} \mathrm{D}_{6}, 25^{\circ} \mathrm{C}\right): \delta 7.49$ (s, 2H, 4/5-imidazolylidene $H$ ), 6.77 (s, 2H, Ar H), 6.74 (s, 2H, Ar H), 6.40 (s, 1H, p-pyr H), 6.33 (d, 2H, 4/5-imidazolylidene $H$ ), 2.86 (s, 6H, Ar- $\left.\mathrm{CH}_{3}\right), 2.21\left(\mathrm{~s}, 6 \mathrm{H}, \mathrm{Ar}-\mathrm{CH}_{3}\right), 2.05$ (s, 6H, m-pyr- $\left.\left(\mathrm{CH}_{3}\right)_{2}\right), 1.94\left(\mathrm{~s}, 6 \mathrm{H}, \mathrm{Ar}-\mathrm{CH}_{3}\right), 0.66$ (s, 9H, Fe-CHDCHDC $\left.\left(\mathrm{CH}_{3}\right)_{3}\right),-0.07(\mathrm{~m}, 1 \mathrm{H}, \mathrm{Fe}-$ 
$\left.\mathrm{CHDCHDC}\left(\mathrm{CH}_{3}\right)_{3}\right),-0.25\left(\mathrm{~m}, 1 \mathrm{H}, \mathrm{Fe}-\mathrm{CHDCHDC}\left(\mathrm{CH}_{3}\right)_{3}\right)$. The complex was sensitive to isolation, so further analytical data were not acquired.

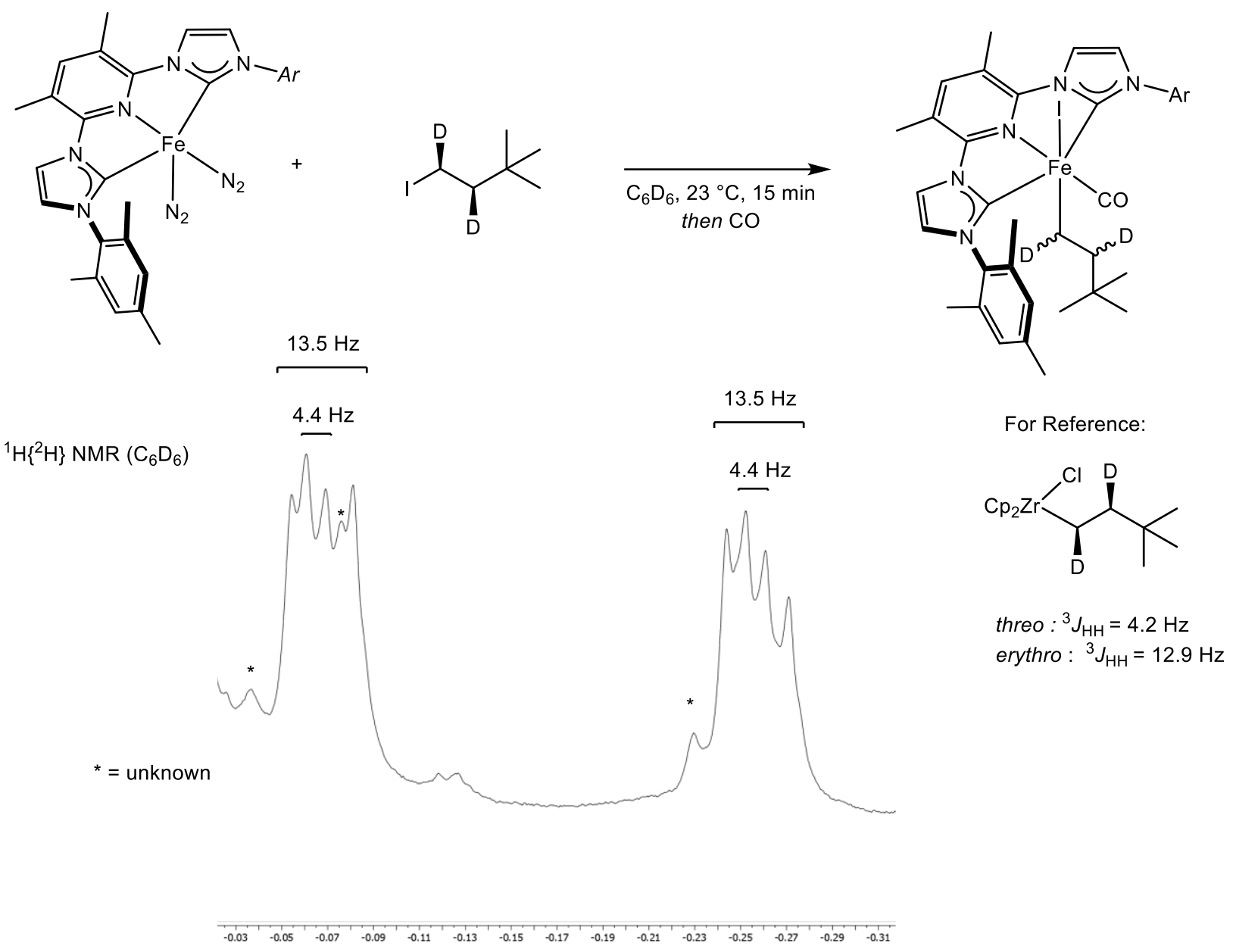

Figure S2. Reaction of $1-\left(\mathrm{N}_{2}\right)_{2}$ with threo-ICHDCHDC $\left(\mathrm{CH}_{3}\right)_{3}$ then subsequent treatment with 0.1 atm $\mathrm{CO}$ at $23{ }^{\circ} \mathrm{C}$ in $\mathrm{C}_{6} \mathrm{D}_{6}$ and subsequent characterization by ${ }^{1} \mathrm{H}\left\{{ }^{2} \mathrm{H}\right\}-\mathrm{NMR}$ in $\mathrm{C}_{6} \mathrm{D}_{6}$ at $25{ }^{\circ} \mathrm{C}$. Reference values for $[\mathrm{Zr}]$ are given. ${ }^{4}$

Reaction of 1-( $\left.\mathbf{N}_{2}\right)_{2}$ with 6-iodo-1-hexene. In an $\mathrm{N}_{2}$-filled glovebox, a $20 \mathrm{~mL}$ scintillation vial was charged with 3,5- $\mathrm{Me}_{2}{ }^{-\mathrm{Mes}} \mathrm{CNCFe}\left(\mathrm{N}_{2}\right)_{2}(29 \mathrm{mg}, 0.049 \mathrm{mmol}, 1.0$ equiv.), toluene (ca. $5 \mathrm{~mL}$ ), and a Teflon-coated stir bar. To the stirring solution was added 6-iodo-1-hexene $(7.1 \mu \mathrm{L}, 0.049 \mathrm{mmol}$, 1.0 equiv.) neat via syringe. After stirring $1 \mathrm{~h}$, pentane $(15 \mathrm{~mL})$ was added, resulting in precipitation of a green solid. The precipitate was isolated via filtration and washed with $3 \times 3 \mathrm{~mL}$ pentane to 
yield $30 \mathrm{mg}(78 \%)$ of a green solid. The solid was characterized by ${ }^{1} \mathrm{H}-\mathrm{NMR}$ in $\mathrm{C}_{6} \mathrm{D}_{6}$ to be the

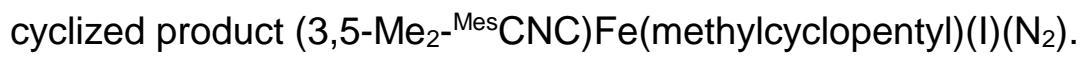

Reaction of 1-(H)( $\left.\mathbf{N}_{2}\right)(\mathbf{I})$ with 1,5-hexadiene. In an $\mathrm{N}_{2}$-filled glovebox, a $20 \mathrm{~mL}$ scintillation vial was charged with (3,5-Me $\left.2^{-}{ }^{M e s} \mathrm{CNC}\right) \mathrm{Fe}(\mathrm{H})\left(\mathrm{N}_{2}\right)(\mathrm{I})\left(10 \mathrm{mg}, 0.014 \mathrm{mmol}, 1.0\right.$ equiv.), $\mathrm{C}_{6} \mathrm{D}_{6}$ (ca. $\left.1 \mathrm{~mL}\right)$, and a Teflon-coated stir bar. To the stirring solution was added 1,5 -hexadiene $(1.7 \mu \mathrm{L}, 0.014$ mmol, 1.0 equiv.) neat via syringe. After stirring $24 \mathrm{~h}$, the solution changed color from brown to green and was filtered. Analysis by ${ }^{1} \mathrm{H}-\mathrm{NMR}$ is consistent with a mixture of $\left(3,5-\mathrm{Me}_{2}-\right.$ $\left.{ }^{\mathrm{Mes}} \mathrm{CNC}\right) \mathrm{Fe}(\mathrm{H})\left(\mathrm{N}_{2}\right)(\mathrm{I})$ and a second [Fe] compound. Vacuum transfer of the volatiles and analysis of the solution by ${ }^{1} \mathrm{H}-\mathrm{NMR}$ showed the presence of 2,4-hexadiene and other organic products. The observation of the isomerized product diene is consistent with insertion.

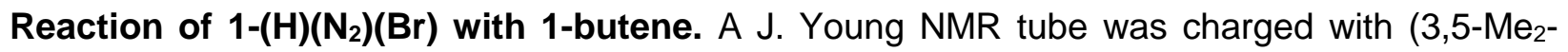
$\left.{ }^{\text {Mes }} \mathrm{CNC}\right) \mathrm{Fe}(\mathrm{H})\left(\mathrm{N}_{2}\right)(\mathrm{Br})\left(10 \mathrm{mg}, 0.016 \mathrm{mmol}, 1.0\right.$ equiv.) and $\mathrm{C}_{6} \mathrm{D}_{6}$ (ca. $\left.0.6 \mathrm{~mL}\right)$. On the vacuum line, the tube was degassed and 1 -butene $(0.016 \mathrm{mmol}, 1.0$ equiv. $)$ was added via calibrated gas bulb at $-196{ }^{\circ} \mathrm{C}$. The solution was then thawed and allowed to stir at room temperature. Upon warming, the solution turned from brown to green. After $1 \mathrm{~h}$, the volatiles were vacuum transferred to new J. Young NMR tube and analyzed by ${ }^{1} \mathrm{H}-\mathrm{NMR}$ tube, showing a mixture of 78:22 E:Z 2butene. 


\section{Reaction of 1-( $\left.\mathrm{N}_{2}\right)_{2}$ with tert-Butyl bromide}
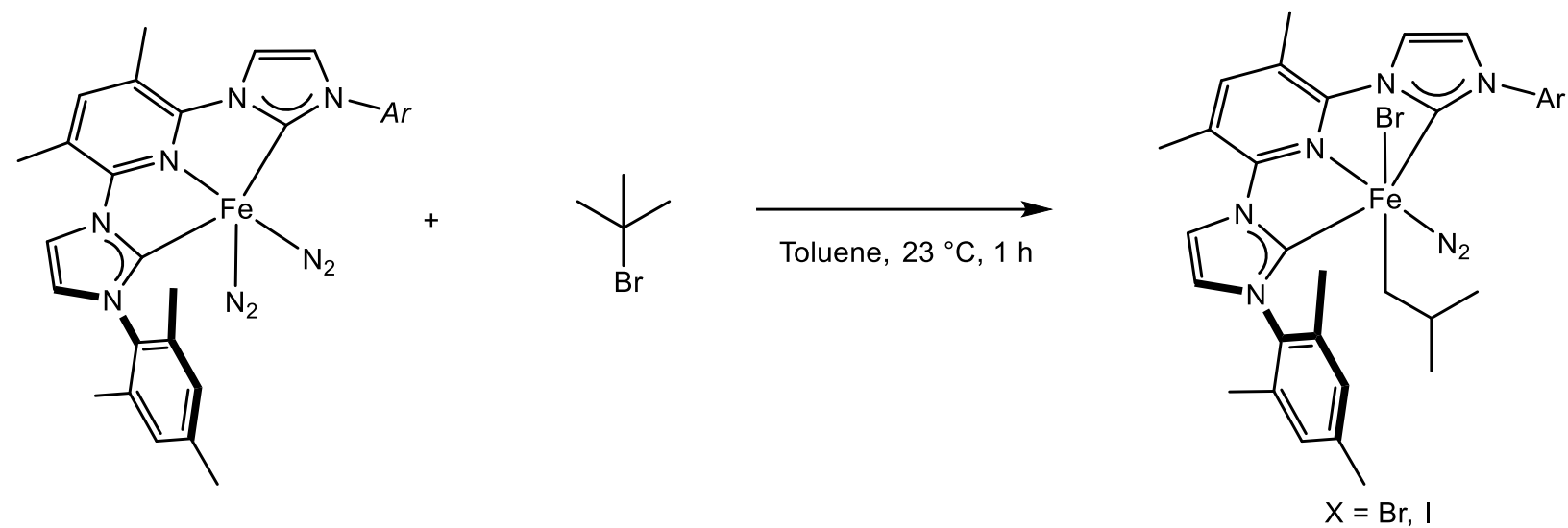

In an $\mathrm{N}_{2}$-filled glovebox, a $20 \mathrm{~mL}$ scintillation vial was charged with $3,5-\mathrm{Me}_{2}{ }^{-}{ }^{-\operatorname{es}} \mathrm{CNCFe}\left(\mathrm{N}_{2}\right)_{2}(50$ $\mathrm{mg}, 0.085 \mathrm{mmol}, 1.0$ equiv.), toluene (ca. $5 \mathrm{~mL}$ ), and a Teflon-coated stir bar. To the stirring solution was added tert-butyl bromide ( $12 \mu \mathrm{L}, 0.10 \mathrm{mmol}, 1.2$ equiv.) neat via syringe. After stirring $1 \mathrm{~h}$, pentane $(15 \mathrm{~mL})$ was added, resulting in precipitation of a green solid. The precipitate was isolated via filtration and washed with $3 \times 3 \mathrm{~mL}$ pentane to yield $46 \mathrm{mg}$ of a green solid identified as $\left(3,5-\mathrm{Me}_{2}{ }^{-\mathrm{Mes}} \mathrm{CNC}\right) \mathrm{Fe}\left(\mathrm{CH}_{2} \mathrm{CHMe}_{2}\right)(\mathrm{Br})\left(\mathrm{N}_{2}\right)$ by ${ }^{1} \mathrm{H}-\mathrm{NMR}$.

\section{Reaction of 1-( $\left.\mathrm{N}_{2}\right)_{2}$ with (bromomethyl)cyclopropane}
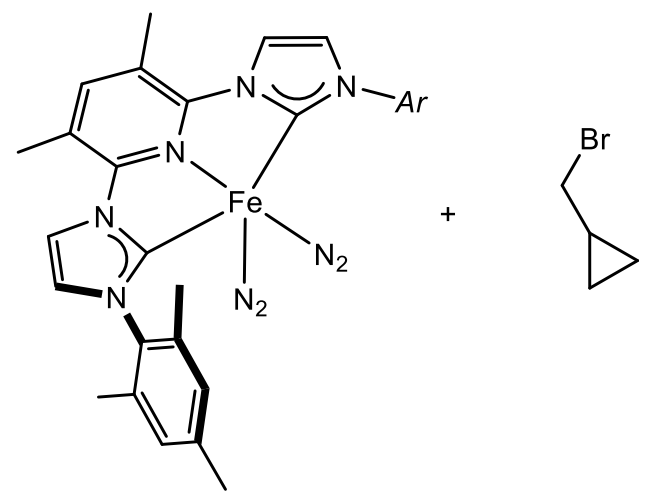

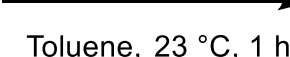

Toluene, $23{ }^{\circ} \mathrm{C}, 1 \mathrm{~h}$

Mixture of products

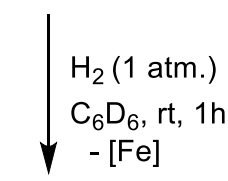

n-butane

In an $\mathrm{N}_{2}$-filled glovebox, a $20 \mathrm{~mL}$ scintillation vial was charged with $3,5-\mathrm{Me}_{2}{ }^{-\operatorname{Mes}} \mathrm{CNCFe}\left(\mathrm{N}_{2}\right)_{2}(18$ $\mathrm{mg}, 0.03 \mathrm{mmol}, 1.0$ equiv.), toluene (ca. $5 \mathrm{~mL}$ ), and a Teflon-coated stir bar. To the stirring solution 
was added (bromomethyl)cyclopropane ( $3 \mu \mathrm{L}, 0.03 \mathrm{mmol}, 1$ equiv.) neat via syringe. After stirring $1 \mathrm{~h}$, pentane $(15 \mathrm{~mL})$ was added, resulting in precipitation of a purple-brown solid. The precipitate was isolated via filtration and washed with $3 \times 3 \mathrm{~mL}$ pentane to yield $16 \mathrm{mg}$ of a green solid. The solid was analyzed by ${ }^{1} \mathrm{H}-\mathrm{NMR}$ in $\mathrm{C}_{6} \mathrm{D}_{6}$ to show a mixture of iron-containing products, so the solution was exposed to 1 atmosphere of $\mathrm{H}_{2}$. Vacuum transfer of the volatiles and analysis by ${ }^{1} \mathrm{H}$ NMR showed only $n$-butane, consistent with formation of the ring-opened product. If the cyclopropane moiety were maintained during reaction, methylcyclopropane would be the expected product upon addition of $\mathrm{H}_{2}$.

Reaction of 1-( $\left.\mathbf{N}_{2}\right)_{2}$ with cyclohexyl bromide. In an $\mathrm{N}_{2}$-filled glovebox, a $20 \mathrm{~mL}$ scintillation vial was charged with (3,5- $\left.\mathrm{Me}_{2}{ }^{\mathrm{Mes}} \mathrm{CNC}\right) \mathrm{Fe}\left(\mathrm{N}_{2}\right)_{2}(30 \mathrm{mg}, 0.051 \mathrm{mmol}, 1.0$ equiv.), toluene (ca. $5 \mathrm{~mL}$ ), and a Teflon-coated stir bar. To the stirring solution was added cyclohexyl bromide $(7 \mu \mathrm{L}, 0.056$ mmol, 1.1 equiv.) neat via syringe. After stirring $1 \mathrm{~h}$, pentane $(15 \mathrm{~mL})$ was added, resulting in precipitation of a purple-brown solid. The precipitate was isolated via filtration and washed with 3 x $3 \mathrm{~mL}$ pentane to yield $28 \mathrm{mg}(86 \%)$ of a purple-brown solid identified as $\left(3,5-\mathrm{Me}_{2}-\right.$ $\left.{ }^{\mathrm{Mes}} \mathrm{CNC}\right) \mathrm{Fe}(\mathrm{H})\left(\mathrm{N}_{2}\right)(\mathrm{Br})$ by ${ }^{1} \mathrm{H}-\mathrm{NMR}$.

\section{Competition Experiments}

\section{Fitting of relative conversions to $k_{\text {rel }}$}

The time course of the competition reactions of two electronically differentiated aryl bromides (Ar1 and Ar2) with 1-( $\left.\mathbf{N}_{2}\right)_{2}$ (Fe) was modeled using COPASI 4.24. The model consists of two competing, irreversible $2^{\text {nd }}$ order reactions which are both $1^{\text {st }}$ order dependent on iron starting material (Fe) and the two aryl halides ( $\mathrm{Ar} 1$ and $\mathrm{Ar} 2)$ to form the two iron products (Fe1 and $\mathrm{Fe} 2)$ (Figure S3). 


\begin{tabular}{c|c|c|c} 
\# & Name & Reaction & Rate Law \\
1 & reaction1 & $\mathrm{Fe}+\mathrm{Ar} 1->\mathrm{Fe} 1$ & Mass action (irreversible) \\
\hline 2 & reaction2 & $\mathrm{Fe}+\mathrm{Ar} 2>\mathrm{Fe} 2$ & Mass action (irreversible) \\
\hline
\end{tabular}

Figure S3. Reactions input in COPASI.

Initial concentrations of $\mathrm{Fe}, \operatorname{Ar} 1$ and $\operatorname{Ar} 2$ were all set to $1 \mathrm{mmol} / \mathrm{mL}$ and $\mathrm{k} 1$ of reaction 1 and 2 were arbitrarily set (Scheme SI 2). The time course was generated to obtain the relative conversion at the end of reaction for the given $\mathrm{k} 1 . \mathrm{k} 1$ of reaction 1 and 2 were manually changed until the calculated relative conversion matched the observed relative conversion of the two aryl halides to obtain $\mathrm{k}_{\mathrm{rel}}=\mathrm{k}_{\mathrm{R} 1} / \mathrm{k}_{\mathrm{R} 2}$.

\begin{tabular}{|c|c|c|c|c|}
\hline \multicolumn{2}{|c|}{ Name } & Type & Value & Unit \\
\hline \multicolumn{5}{|c|}{$\checkmark$ Initial Time } \\
\hline & New Model & time & 0 & s \\
\hline \multicolumn{5}{|c|}{$\checkmark$ Initial Compartment Sizes } \\
\hline & compartment & fixed & 1 & $\mathrm{ml}$ \\
\hline \multirow[t]{7}{*}{$\checkmark$} & Initial Species Values & & & \\
\hline & $\mathrm{Fe}$ & reactions & 1 & $\mathrm{mmol} / \mathrm{ml}$ \\
\hline & Ar1 & reactions & 1 & $\mathrm{mmol} / \mathrm{ml}$ \\
\hline & $\operatorname{Ar} 2$ & reactions & 1 & $\mathrm{mmol} / \mathrm{ml}$ \\
\hline & $\mathrm{Fe} 1$ & reactions & 0 & $\mathrm{mmol} / \mathrm{ml}$ \\
\hline & $\mathrm{Fe} 2$ & reactions & 0 & $\mathrm{mmol} / \mathrm{ml}$ \\
\hline & Initial Global Quantities & & & \\
\hline \multirow[t]{5}{*}{$\checkmark$} & Kinetic Parameters & & & \\
\hline & $\checkmark$ reaction 1 & & & $\mathrm{mmol} /\left(\mathrm{ml}^{\star} \mathrm{s}\right)$ \\
\hline & k1 & fixed & 2,2 & $\mathrm{ml} /\left(\mathrm{mmol}^{\star} \mathrm{s}\right)$ \\
\hline & $\checkmark$ reaction 2 & & & $\mathrm{mmol} /\left(\mathrm{ml}^{*} \mathrm{~s}\right)$ \\
\hline & k1 & fixed & 1 & $\mathrm{ml} /\left(\mathrm{mmol}^{*} \mathrm{~s}\right)$ \\
\hline
\end{tabular}

Figure S4. Set model parameters in COPASI.

Fitted time course for 4-F aryl bromide and 4-OMe aryl bromide

Input: k1 (reaction 1): 4.8; k1 (reaction 2): 1

$\begin{array}{cccccl}\text { \# } & \text { Time Fe } & \text { Ar1 } & \text { Ar2 } & \text { Fe1 } & \text { Fe2 } \\ 0 & 1 & 1 & 1 & 0 & 0\end{array}$




\begin{tabular}{|c|c|c|c|c|c|}
\hline 1 & 0.0551528 & 0.285171 & 0.769982 & 0.714829 & 0.230018 \\
\hline 2 & 0.00724029 & 0.254994 & 0.752247 & 0.745006 & 0.247753 \\
\hline 3 & 0.00101724 & 0.251148 & 0.749869 & 0.748852 & 0.250131 \\
\hline 4 & 0.000144225 & 0.25061 & 0.749534 & 0.74939 & 0.250466 \\
\hline 5 & $2.04746 \mathrm{e}-05$ & 0.250534 & 0.749487 & 0.749466 & 0.250513 \\
\hline 6 & $2.90715 \mathrm{e}-06$ & 0.250523 & 0.74948 & 0.749477 & 0.25052 \\
\hline 7 & 4.12792e-07 & 0.250521 & 0.749479 & 0.749479 & 0.250521 \\
\hline 8 & $5.86133 e-08$ & 0.250521 & 0.749479 & 0.749479 & 0.250521 \\
\hline 9 & 8.32258e-09 & 0.250521 & 0.749479 & 0.749479 & 0.250521 \\
\hline 10 & $1.18182 \mathrm{e}-09$ & 0.250521 & 0.749479 & 0.749479 & 0.250521 \\
\hline 11 & $1.67809 \mathrm{e}-10$ & 0.250521 & 0.749479 & 0.749479 & 0.250521 \\
\hline 12 & $2.38478 \mathrm{e}-11$ & 0.250521 & 0.749479 & 0.749479 & 0.250521 \\
\hline 13 & 3.34897e-12 & 0.250521 & 0.749479 & 0.749479 & 0.250521 \\
\hline 14 & $4.86052 e-13$ & 0.250521 & 0.749479 & 0.749479 & 0.250521 \\
\hline 15 & $7.40751 \mathrm{e}-14$ & 0.250521 & 0.749479 & 0.749479 & 0.250521 \\
\hline 16 & $-1.15051 e-14$ & 0.250521 & 0.749479 & 0.749479 & 0.250521 \\
\hline 17 & $-2.54759 e-14$ & 0.250521 & 0.749479 & 0.749479 & 0.250521 \\
\hline 18 & $-2.3716 e-14$ & 0.250521 & 0.749479 & 0.749479 & 0.250521 \\
\hline 19 & $-1.10332 e-14$ & 0.250521 & 0.749479 & 0.749479 & 0.250521 \\
\hline 20 & $-5.4881 e-15$ & 0.250521 & 0.749479 & 0.749479 & 0.250521 \\
\hline 21 & $-2.75453 e-15$ & 0.250521 & 0.749479 & 0.749479 & 0.250521 \\
\hline 22 & $-2.58949 e-15$ & 0.250521 & 0.749479 & 0.749479 & 0.250521 \\
\hline 23 & $-2.42444 \mathrm{e}-15$ & 0.250521 & 0.749479 & 0.749479 & 0.250521 \\
\hline 24 & $-2.2594 e-15$ & 0.250521 & 0.749479 & 0.749479 & 0.250521 \\
\hline 25 & $-2.09436 e-15$ & 0.250521 & 0.749479 & 0.749479 & 0.250521 \\
\hline 26 & $-1.92932 e-15$ & 0.250521 & 0.749479 & 0.749479 & 0.250521 \\
\hline 27 & $-1.76427 e-15$ & 0.250521 & 0.749479 & 0.749479 & 0.250521 \\
\hline 28 & $-1.59923 e-15$ & 0.250521 & 0.749479 & 0.749479 & 0.250521 \\
\hline 29 & $-1.43419 e-15$ & 0.250521 & 0.749479 & 0.749479 & 0.250521 \\
\hline 30 & $-1.26915 e-15$ & 0.250521 & 0.749479 & 0.749479 & 0.250521 \\
\hline 31 & $-1.1041 e-15$ & 0.250521 & 0.749479 & 0.749479 & 0.250521 \\
\hline 32 & $-9.39061 e-16$ & 0.250521 & 0.749479 & 0.749479 & 0.250521 \\
\hline 33 & $-7.74019 e-16$ & 0.250521 & 0.749479 & 0.749479 & 0.250521 \\
\hline 34 & $-6.08976 e-16$ & 0.250521 & 0.749479 & 0.749479 & 0.250521 \\
\hline 35 & $-4.43934 e-16$ & 0.250521 & 0.749479 & 0.749479 & 0.250521 \\
\hline 36 & $-2.78891 e-16$ & 0.250521 & 0.749479 & 0.749479 & 0.250521 \\
\hline 37 & $-1.13849 e-16$ & 0.250521 & 0.749479 & 0.749479 & 0.250521 \\
\hline 38 & $-8.05111 e-17$ & 0.250521 & 0.749479 & 0.749479 & 0.250521 \\
\hline 39 & $-7.55988 e-17$ & 0.250521 & 0.749479 & 0.749479 & 0.250521 \\
\hline 40 & $-7.06866 e-17$ & 0.250521 & 0.749479 & 0.749479 & 0.250521 \\
\hline 41 & $-6.57744 e-17$ & 0.250521 & 0.749479 & 0.749479 & 0.250521 \\
\hline 42 & $-6.08621 e-17$ & 0.250521 & 0.749479 & 0.749479 & 0.250521 \\
\hline 43 & $-5.59499 e-17$ & 0.250521 & 0.749479 & 0.749479 & 0.250521 \\
\hline 44 & $-5.10377 e-17$ & 0.250521 & 0.749479 & 0.749479 & 0.250521 \\
\hline
\end{tabular}




\begin{tabular}{|c|c|c|c|c|c|}
\hline 45 & $-4.61254 e-17$ & 0.250521 & 0.749479 & 0.749479 & 0.250521 \\
\hline 46 & $-4.12132 e-17$ & 0.250521 & 0.749479 & 0.749479 & 0.250521 \\
\hline 47 & $-3.6301 e-17$ & 0.250521 & 0.749479 & 0.749479 & 0.250521 \\
\hline 48 & $-3.13888 e-17$ & 0.250521 & 0.749479 & 0.749479 & 0.250521 \\
\hline 49 & $-2.64765 e-17$ & 0.250521 & 0.749479 & 0.749479 & 0.250521 \\
\hline 50 & $-2.15643 e-17$ & 0.250521 & 0.749479 & 0.749479 & 0.250521 \\
\hline 51 & $-1.66521 e-17$ & 0.250521 & 0.749479 & 0.749479 & 0.250521 \\
\hline 52 & $-1.17398 e-17$ & 0.250521 & 0.749479 & 0.749479 & 0.250521 \\
\hline 53 & $-6.82761 e-18$ & 0.250521 & 0.749479 & 0.749479 & 0.250521 \\
\hline 54 & $-2.5147 e-18$ & 0.250521 & 0.749479 & 0.749479 & 0.25 \\
\hline 55 & $-2.49967 e-18$ & 0.250521 & 0.749479 & 0.749479 & 521 \\
\hline 56 & $-2.48465 e-18$ & 0.250521 & 0.749479 & 0.749479 & 0.2 \\
\hline 57 & $-2.46963 e-18$ & 0.250521 & 0.749479 & 0.749479 & 0.2 \\
\hline 58 & $-2.45461 e-18$ & 0.250521 & 0.749479 & 0.749479 & 0.2 \\
\hline 59 & $-2.43958 e-18$ & 0.250521 & 0.749479 & 0.749479 & 0.2 \\
\hline 60 & $-2.42456 e-18$ & 0.250521 & 0.749479 & 0.749479 & 0.250521 \\
\hline 61 & $-2.40954 e-18$ & 0.250521 & 0.749479 & 0.749479 & 0.2 \\
\hline 62 & $-2.39451 e-18$ & 0.250521 & 0.749479 & 0.749479 & 0.2 \\
\hline 63 & $-2.37949 e-18$ & 0.250521 & 0.749479 & 0.749479 & 0.250521 \\
\hline 64 & $-2.36447 e-18$ & 0.250521 & 0.749479 & 0.749479 & \\
\hline 65 & $-2.34945 e-18$ & 0.250521 & 0.749479 & 0.749479 & 0.250521 \\
\hline 66 & $-2.33442 e-18$ & 0.250521 & 0.749479 & 0.749479 & 521 \\
\hline 67 & $-2.3194 e-18$ & 0.250521 & 0.749479 & 0.749479 & 0.2 \\
\hline 68 & $-2.30438 e-18$ & 0.250521 & 0.749479 & 0.749479 & 0.250521 \\
\hline 69 & $-2.28935 e-18$ & 0.250521 & 0.749479 & 0.749479 & 0521 \\
\hline 70 & $-2.27433 e-18$ & 0.250521 & 0.749479 & 0.749479 & 0521 \\
\hline 71 & $-2.25931 e-18$ & 0.250521 & 0.749479 & 0.749479 & 0.250521 \\
\hline 72 & $-2.24429 e-18$ & 0.250521 & 0.749479 & 0.749479 & 0521 \\
\hline 73 & $-2.22926 e-18$ & 0.250521 & 0.749479 & 0.749479 & 0.250521 \\
\hline 74 & $-2.21424 e-18$ & 0.250521 & 0.749479 & 0.749479 & 0.25 \\
\hline 75 & $-2.19922 \mathrm{e}-18$ & 0.250521 & 0.749479 & 0.749479 & 0.250521 \\
\hline 76 & $-2.18419 e-18$ & 0.250521 & 0.749479 & 0.749479 & 0.250521 \\
\hline 77 & $-2.16917 e-18$ & 0.250521 & 0.749479 & 0.749479 & 0.250521 \\
\hline 78 & $-2.15415 e-18$ & 0.250521 & 0.749479 & 0.749479 & 0.250521 \\
\hline 79 & $-2.13913 e-18$ & 0.250521 & 0.749479 & 0.749479 & 0.250521 \\
\hline 80 & $-2.1241 e-18$ & 0.250521 & 0.749479 & 0.749479 & 0.250521 \\
\hline 81 & $-2.10908 e-18$ & 0.250521 & 0.749479 & 0.749479 & 0.250521 \\
\hline 82 & $-2.09406 e-18$ & 0.250521 & 0.749479 & 0.749479 & 0.250521 \\
\hline 83 & $-2.07903 e-18$ & 0.250521 & 0.749479 & 0.749479 & 0.250521 \\
\hline 84 & $-2.06401 e-18$ & 0.250521 & 0.749479 & 0.749479 & 0.25052 \\
\hline 85 & $-2.04899 e-18$ & 0.250521 & 0.749479 & 0.749479 & 0.250521 \\
\hline 86 & $-2.03397 e-18$ & 0.250521 & 0.749479 & 0.749479 & 0.250521 \\
\hline 87 & $-2.01894 e-18$ & 0.250521 & 0.749479 & 0.749479 & 0.25052 \\
\hline 88 & $-2.00392 e-18$ & 0.250521 & 0.749479 & 0.749479 & 0.25052 \\
\hline
\end{tabular}




$\begin{array}{llllll}89 & -1.9889 \mathrm{e}-18 & 0.250521 & 0.749479 & 0.749479 & 0.250521 \\ 90 & -1.97387 \mathrm{e}-18 & 0.250521 & 0.749479 & 0.749479 & 0.250521 \\ 91 & -1.95885 \mathrm{e}-18 & 0.250521 & 0.749479 & 0.749479 & 0.250521 \\ 92 & -1.94383 \mathrm{e}-18 & 0.250521 & 0.749479 & 0.749479 & 0.250521 \\ 93 & -1.9288 \mathrm{e}-18 & 0.250521 & 0.749479 & 0.749479 & 0.250521 \\ 94 & -1.91378 \mathrm{e}-18 & 0.250521 & 0.749479 & 0.749479 & 0.250521 \\ 95 & -1.89876 \mathrm{e}-18 & 0.250521 & 0.749479 & 0.749479 & 0.250521 \\ 96 & -1.88374 \mathrm{e}-18 & 0.250521 & 0.749479 & 0.749479 & 0.250521 \\ 97 & -1.86871 \mathrm{e}-18 & 0.250521 & 0.749479 & 0.749479 & 0.250521 \\ 98 & -1.85369 \mathrm{e}-18 & 0.250521 & 0.749479 & 0.749479 & 0.250521 \\ 99 & -1.83867 \mathrm{e}-18 & 0.250521 & 0.749479 & 0.749479 & 0.250521 \\ 100 & -1.82364 \mathrm{e}-18 & 0.250521 & 0.749479 & 0.749479 & 0.250521\end{array}$

Fitted time course for 4- $\mathrm{H}$ aryl bromide and 4-OMe aryl bromide

Input: k1 (reaction 1): 2.2; k1 (reaction 2): 1

$\begin{array}{llllll}\text { \# Time Fe } & \text { Ar1 } & \text { Ar2 } & \text { Fe1 } & \text { Fe2 } \\ 0 & 1 & 1 & 1 & 0 & 0 \\ 1 & 0.124028 & 0.437361 & 0.686666 & 0.562639 & 0.313334 \\ 2 & 0.0264703 & 0.381306 & 0.645164 & 0.618694 & 0.354836 \\ 3 & 0.00612951 & 0.36985 & 0.63628 & 0.63015 & 0.36372 \\ 4 & 0.00144484 & 0.367223 & 0.634222 & 0.632777 & 0.365778 \\ 5 & 0.000341989 & 0.366605 & 0.633736 & 0.633395 & 0.366264 \\ 6 & 8.10268 \mathrm{e}-05 & 0.366459 & 0.633622 & 0.633541 & 0.366378 \\ 7 & 1.9202 \mathrm{e}-05 & 0.366425 & 0.633594 & 0.633575 & 0.366406 \\ 8 & 4.55078 \mathrm{e}-06 & 0.366417 & 0.633588 & 0.633583 & 0.366412 \\ 9 & 1.07853 \mathrm{e}-06 & 0.366415 & 0.633586 & 0.633585 & 0.366414 \\ 10 & 2.55611 \mathrm{e}-07 & 0.366414 & 0.633586 & 0.633586 & 0.366414 \\ 11 & 6.05795 \mathrm{e}-08 & 0.366414 & 0.633586 & 0.633586 & 0.366414 \\ 12 & 1.43573 \mathrm{e}-08 & 0.366414 & 0.633586 & 0.633586 & 0.366414 \\ 13 & 3.40273 \mathrm{e}-09 & 0.366414 & 0.633586 & 0.633586 & 0.366414 \\ 14 & 8.06448 \mathrm{e}-10 & 0.366414 & 0.633586 & 0.633586 & 0.366414 \\ 15 & 1.91135 \mathrm{e}-10 & 0.366414 & 0.633586 & 0.633586 & 0.366414 \\ 16 & 4.52307 \mathrm{e}-11 & 0.366414 & 0.633586 & 0.633586 & 0.366414 \\ 17 & 1.08431 \mathrm{e}-11 & 0.366414 & 0.633586 & 0.633586 & 0.366414 \\ 18 & 2.48155 \mathrm{e}-12 & 0.366414 & 0.633586 & 0.633586 & 0.366414 \\ 19 & 6.08112 \mathrm{e}-13 & 0.366414 & 0.633586 & 0.633586 & 0.366414 \\ 20 & 1.52501 \mathrm{e}-13 & 0.366414 & 0.633586 & 0.633586 & 0.366414 \\ 21 & 3.80061 \mathrm{e}-14 & 0.366414 & 0.633586 & 0.633586 & 0.366414 \\ 22 & -2.22202 \mathrm{e}-14 & 0.366414 & 0.633586 & 0.633586 & 0.366414 \\ 23 & -2.61867 \mathrm{e}-14 & 0.366414 & 0.633586 & 0.633586 & 0.366414 \\ 24 & -2.7117 \mathrm{e}-14 & 0.366414 & 0.633586 & 0.633586 & 0.366414\end{array}$




\begin{tabular}{|c|c|c|c|c|c|}
\hline 25 & $-1.85818 e-14$ & 0.366414 & 0.633586 & 0.633586 & 0.366414 \\
\hline 26 & $-1.09148 e-14$ & 0.366414 & 0.633586 & 0.633586 & 0.366414 \\
\hline 27 & $-5.6604 e-15$ & 0.366414 & 0.633586 & 0.633586 & 0.366414 \\
\hline 28 & $-3.54691 e-15$ & 0.366414 & 0.633586 & 0.633586 & 0.366414 \\
\hline 29 & $-3.38045 e-15$ & 0.366414 & 0.633586 & 0.633586 & 0.366414 \\
\hline 30 & $-3.214 e-15$ & 0.366414 & 0.633586 & 0.633586 & 0.366414 \\
\hline 31 & $-3.04755 e-15$ & 0.366414 & 0.633586 & 0.633586 & 0.366414 \\
\hline 32 & $-2.8811 e-15$ & 0.366414 & 0.633586 & 0.633586 & 0.366414 \\
\hline 33 & $-2.71465 e-15$ & 0.366414 & 0.633586 & 0.633586 & 0.366414 \\
\hline 34 & $-2.5482 e-15$ & 0.366414 & 0.633586 & 0.633586 & 0.366414 \\
\hline 35 & $-2.38175 e-15$ & 0.366414 & 0.633586 & 0.633586 & 0.366414 \\
\hline 36 & $-2.2153 e-15$ & 0.366414 & 0.633586 & 0.633586 & 0.366414 \\
\hline 37 & $-2.04884 e-15$ & 0.366414 & 0.633586 & 0.633586 & 0.366414 \\
\hline 38 & $-1.88239 e-15$ & 0.366414 & 0.633586 & 0.633586 & 0.366414 \\
\hline 39 & $-1.71594 e-15$ & 0.366414 & 0.633586 & 0.633586 & \\
\hline 40 & $-1.54949 e-15$ & 0.366414 & 0.633586 & 0.633586 & 0.366414 \\
\hline 41 & $-1.38304 e-15$ & 0.366414 & 0.633586 & 0.633586 & 0.366414 \\
\hline 42 & $-1.21659 e-15$ & 0.366414 & 0.633586 & 0.633586 & 0.366414 \\
\hline 43 & $-1.05014 e-15$ & 0.366414 & 0.633586 & 0.633586 & 0.366414 \\
\hline 44 & $-8.83685 e-16$ & 0.366414 & 0.633586 & 0.633586 & 0.366414 \\
\hline 45 & $-7.17234 \mathrm{e}-16$ & 0.366414 & 0.633586 & 0.633586 & 0.366414 \\
\hline 46 & $-5.50783 e-16$ & 0.366414 & 0.633586 & 0.633586 & 0.366414 \\
\hline 47 & $-3.84332 e-16$ & 0.366414 & 0.633586 & 0.633586 & \\
\hline 48 & $-2.1788 e-16$ & 0.366414 & 0.633586 & 0.633586 & 0.366414 \\
\hline 49 & $-1.13582 e-16$ & 0.366414 & 0.633586 & 0.633586 & 0.366414 \\
\hline 50 & $-1.08309 e-16$ & 0.366414 & 0.633586 & 0.633586 & 0.366414 \\
\hline 51 & $-1.03036 e-16$ & 0.366414 & 0.633586 & 0.633586 & 0.366414 \\
\hline 52 & $-9.77634 e-17$ & 0.366414 & 0.633586 & 0.633586 & 0.366414 \\
\hline 53 & $-9.24905 e-17$ & 0.366414 & 0.633586 & 0.633586 & 0.366414 \\
\hline 54 & $-8.72176 e-17$ & 0.366414 & 0.633586 & 0.633586 & 0.366414 \\
\hline 55 & $-8.19447 e-17$ & 0.366414 & 0.633586 & 0.633586 & 0.366414 \\
\hline 56 & $-7.66718 e-17$ & 0.366414 & 0.633586 & 0.633586 & 0.366414 \\
\hline 57 & $-7.13988 e-17$ & 0.366414 & 0.633586 & 0.633586 & 0.366414 \\
\hline 58 & $-6.61259 e-17$ & 0.366414 & 0.633586 & 0.633586 & 0.366414 \\
\hline 59 & $-6.0853 e-17$ & 0.366414 & 0.633586 & 0.633586 & 0.366414 \\
\hline 60 & $-5.55801 e-17$ & 0.366414 & 0.633586 & 0.633586 & 0.366414 \\
\hline 61 & $-5.03072 e-17$ & 0.366414 & 0.633586 & 0.633586 & 0.366414 \\
\hline 62 & $-4.50343 e-17$ & 0.366414 & 0.633586 & 0.633586 & 0.366414 \\
\hline 63 & $-3.97614 e-17$ & 0.366414 & 0.633586 & 0.633586 & 0.366414 \\
\hline 64 & $-3.44884 e-17$ & 0.366414 & 0.633586 & 0.633586 & 0.366414 \\
\hline 65 & $-2.92155 e-17$ & 0.366414 & 0.633586 & 0.633586 & 0.366414 \\
\hline 66 & $-2.39426 e-17$ & 0.366414 & 0.633586 & 0.633586 & 0.366414 \\
\hline 67 & $-1.86697 e-17$ & 0.366414 & 0.633586 & 0.633586 & 0.366414 \\
\hline 68 & $-1.33968 e-17$ & 0.366414 & 0.633586 & 0.633586 & 0.366414 \\
\hline
\end{tabular}




$\begin{array}{llllll}69 & -8.12388 \mathrm{e}-18 & 0.366414 & 0.633586 & 0.633586 & 0.366414 \\ 70 & -3.65987 \mathrm{e}-18 & 0.366414 & 0.633586 & 0.633586 & 0.366414 \\ 71 & -3.64268 \mathrm{e}-18 & 0.366414 & 0.633586 & 0.633586 & 0.366414 \\ 72 & -3.62548 \mathrm{e}-18 & 0.366414 & 0.633586 & 0.633586 & 0.366414 \\ 73 & -3.60829 \mathrm{e}-18 & 0.366414 & 0.633586 & 0.633586 & 0.366414 \\ 74 & -3.59109 \mathrm{e}-18 & 0.366414 & 0.633586 & 0.633586 & 0.366414 \\ 75 & -3.5739 \mathrm{e}-18 & 0.366414 & 0.633586 & 0.633586 & 0.366414 \\ 76 & -3.55671 \mathrm{e}-18 & 0.366414 & 0.633586 & 0.633586 & 0.366414 \\ 77 & -3.53951 \mathrm{e}-18 & 0.366414 & 0.633586 & 0.633586 & 0.366414 \\ 78 & -3.52232 \mathrm{e}-18 & 0.366414 & 0.633586 & 0.633586 & 0.366414 \\ 79 & -3.50512 \mathrm{e}-18 & 0.366414 & 0.633586 & 0.633586 & 0.366414 \\ 80 & -3.48793 \mathrm{e}-18 & 0.366414 & 0.633586 & 0.633586 & 0.366414 \\ 81 & -3.47074 \mathrm{e}-18 & 0.366414 & 0.633586 & 0.633586 & 0.366414 \\ 82 & -3.45354 \mathrm{e}-18 & 0.366414 & 0.633586 & 0.633586 & 0.366414 \\ 83 & -3.43635 \mathrm{e}-18 & 0.366414 & 0.633586 & 0.633586 & 0.366414 \\ 84 & -3.41915 \mathrm{e}-18 & 0.366414 & 0.633586 & 0.633586 & 0.366414 \\ 85 & -3.40196 \mathrm{e}-18 & 0.366414 & 0.633586 & 0.633586 & 0.366414 \\ 86 & -3.38477 \mathrm{e}-18 & 0.366414 & 0.633586 & 0.633586 & 0.366414 \\ 87 & -3.36757 \mathrm{e}-18 & 0.366414 & 0.633586 & 0.633586 & 0.366414 \\ 88 & -3.35038 \mathrm{e}-18 & 0.366414 & 0.633586 & 0.633586 & 0.366414 \\ 89 & -3.33319 \mathrm{e}-18 & 0.366414 & 0.633586 & 0.633586 & 0.366414 \\ 90 & -3.31599 \mathrm{e}-18 & 0.366414 & 0.633586 & 0.633586 & 0.366414 \\ 91 & -3.2988 \mathrm{e}-18 & 0.366414 & 0.633586 & 0.633586 & 0.366414 \\ 92 & -3.2816 \mathrm{e}-18 & 0.366414 & 0.633586 & 0.633586 & 0.366414 \\ 93 & -3.26441 \mathrm{e}-18 & 0.366414 & 0.633586 & 0.633586 & 0.366414 \\ 94 & -3.24722 \mathrm{e}-18 & 0.366414 & 0.633586 & 0.633586 & 0.366414 \\ 95 & -3.23002 \mathrm{e}-18 & 0.366414 & 0.633586 & 0.633586 & 0.366414 \\ 96 & -3.21283 \mathrm{e}-18 & 0.366414 & 0.633586 & 0.633586 & 0.366414 \\ 97 & -3.19563 \mathrm{e}-18 & 0.366414 & 0.633586 & 0.633586 & 0.366414 \\ 98 & -3.17844 \mathrm{e}-18 & 0.366414 & 0.633586 & 0.633586 & 0.366414 \\ 99 & -3.16125 \mathrm{e}-18 & 0.366414 & 0.633586 & 0.633586 & 0.366414 \\ 100 & -3.14405 \mathrm{e}-18 & 0.366414 & 0.633586 & 0.633586 & 0.366414\end{array}$

Fitted time course for 4-CF3 aryl bromide and 4-F aryl bromide

Input: k1 (reaction 1): 3.2; k1 (reaction 2): 1

$\begin{array}{llllll}\text { \# } & \text { Time Fe } & \text { Ar1 } & \text { Ar2 } & \text { Fe1 } & \text { Fe2 } \\ 0 & 1 & 1 & 1 & 0 & 0 \\ 1 & 0.0862164 & 0.359714 & 0.726502 & 0.640286 & 0.273498 \\ 2 & 0.0146915 & 0.316602 & 0.698089 & 0.683398 & 0.301911 \\ 3 & 0.00270091 & 0.309526 & 0.693175 & 0.690474 & 0.306825 \\ 4 & 0.000503148 & 0.308234 & 0.692269 & 0.691766 & 0.307731 \\ 5 & 9.39592 \mathrm{e}-05 & 0.307993 & 0.692101 & 0.692007 & 0.307899\end{array}$




\begin{tabular}{|c|c|c|c|c|c|}
\hline 6 & $1.75542 \mathrm{e}-05$ & 0.307948 & 0.692069 & 0.692052 & 0.307931 \\
\hline 7 & $3.27989 e-06$ & 0.30794 & 0.692063 & 0.69206 & 0.307937 \\
\hline 8 & $6.12836 \mathrm{e}-07$ & 0.307938 & 0.692062 & 0.692062 & 0.307938 \\
\hline 9 & $1.14507 e-07$ & 0.307938 & 0.692062 & 0.692062 & 0.307938 \\
\hline 10 & 2.13952e-08 & 0.307938 & 0.692062 & 0.692062 & 0.307938 \\
\hline 11 & $3.99764 \mathrm{e}-09$ & 0.307938 & 0.692062 & 0.692062 & 0.307938 \\
\hline 12 & $7.46985 e-10$ & 0.307938 & 0.692062 & 0.692062 & 0.307938 \\
\hline 13 & $1.39557 \mathrm{e}-10$ & 0.307938 & 0.692062 & 0.692062 & 0.307938 \\
\hline 14 & $2.60738 \mathrm{e}-11$ & 0.307938 & 0.692062 & 0.692062 & 0.307938 \\
\hline 15 & $4.92308 e-12$ & 0.307938 & 0.692062 & 0.692062 & 0.307938 \\
\hline 16 & $8.78402 e-13$ & 0.307938 & 0.692062 & 0.692062 & 0.307938 \\
\hline 17 & $1.76559 \mathrm{e}-13$ & 0.307938 & 0.692062 & 0.692062 & 0.307938 \\
\hline 18 & $3.47058 e-14$ & 0.307938 & 0.692062 & 0.692062 & 0.307938 \\
\hline 19 & $-2.38053 e-14$ & 0.307938 & 0.692062 & 0.692062 & 0.307938 \\
\hline 20 & $-2.092 e-14$ & 0.307938 & 0.692062 & 0.692062 & 0.307938 \\
\hline 21 & $-2.38544 e-14$ & 0.307938 & 0.692062 & 0.692062 & 0.307938 \\
\hline 22 & $-1.19135 e-14$ & 0.307938 & 0.692062 & 0.692062 & 0.307938 \\
\hline 23 & $-7.02946 e-15$ & 0.307938 & 0.692062 & 0.692062 & 0.307938 \\
\hline 24 & $-2.88852 e-15$ & 0.307938 & 0.692062 & 0.692062 & 0.307938 \\
\hline 25 & $-2.74167 e-15$ & 0.307938 & 0.692062 & 0.692062 & 0.307938 \\
\hline 26 & $-2.59482 e-15$ & 0.307938 & 0.692062 & 0.692062 & 0.307938 \\
\hline 27 & $-2.44796 e-15$ & 0.307938 & 0.692062 & 0.692062 & 0.307938 \\
\hline 28 & $-2.30111 e-15$ & 0.307938 & 0.692062 & 0.692062 & 0.307938 \\
\hline 29 & $-2.15426 e-15$ & 0.307938 & 0.692062 & 0.692062 & 0.307938 \\
\hline 30 & $-2.0074 e-15$ & 0.307938 & 0.692062 & 0.692062 & 0.307938 \\
\hline 31 & $-1.86055 e-15$ & 0.307938 & 0.692062 & 0.692062 & 0.307938 \\
\hline 32 & $-1.7137 e-15$ & 0.307938 & 0.692062 & 0.692062 & 0.307938 \\
\hline 33 & $-1.56684 e-15$ & 0.307938 & 0.692062 & 0.692062 & 0.307938 \\
\hline 34 & $-1.41999 e-15$ & 0.307938 & 0.692062 & 0.692062 & 0.307938 \\
\hline 35 & $-1.27313 e-15$ & 0.307938 & 0.692062 & 0.692062 & 0.307938 \\
\hline 36 & $-1.12628 e-15$ & 0.307938 & 0.692062 & 0.692062 & 0.307938 \\
\hline 37 & $-9.79427 e-16$ & 0.307938 & 0.692062 & 0.692062 & 0.307938 \\
\hline 38 & $-8.32574 e-16$ & 0.307938 & 0.692062 & 0.692062 & 0.307938 \\
\hline 39 & $-6.8572 e-16$ & 0.307938 & 0.692062 & 0.692062 & 0.307938 \\
\hline 40 & $-5.38867 e-16$ & 0.307938 & 0.692062 & 0.692062 & 0.307938 \\
\hline 41 & $-3.92013 e-16$ & 0.307938 & 0.692062 & 0.692062 & 0.307938 \\
\hline 42 & $-2.4516 e-16$ & 0.307938 & 0.692062 & 0.692062 & 0.307938 \\
\hline 43 & $-9.83061 e-17$ & 0.307938 & 0.692062 & 0.692062 & 0.307938 \\
\hline 44 & $-8.34529 e-17$ & 0.307938 & 0.692062 & 0.692062 & 0.307938 \\
\hline 45 & $-7.90373 e-17$ & 0.307938 & 0.692062 & 0.692062 & 0.307938 \\
\hline 46 & $-7.46217 e-17$ & 0.307938 & 0.692062 & 0.692062 & 0.307938 \\
\hline 47 & $-7.02061 e-17$ & 0.307938 & 0.692062 & 0.692062 & 0.307938 \\
\hline 48 & $-6.57905 e-17$ & 0.307938 & 0.692062 & 0.692062 & 0.307938 \\
\hline 49 & $-6.13749 e-17$ & 0.307938 & 0.692062 & 0.692062 & 0.30793 \\
\hline
\end{tabular}




\begin{tabular}{|c|c|c|c|c|}
\hline$-5.69593 e-17$ & 0.307938 & 0.692062 & 0.692062 & 0.307938 \\
\hline$-5.25437 e-17$ & 0.307938 & 0.692062 & 0.692062 & 0.307938 \\
\hline$-4.81281 e-17$ & 0.307938 & 0.692062 & 0.692062 & 0.307938 \\
\hline$-4.37125 e-17$ & 0.307938 & 0.692062 & 0.692062 & 0.307938 \\
\hline$-3.92969 e-17$ & 0.307938 & 0.692062 & 0.692062 & 0.307938 \\
\hline$-3.48813 e-17$ & 0.307938 & 0.692062 & 0.692062 & 0.307938 \\
\hline$-3.04657 e-17$ & 0.307938 & 0.692062 & 0.692062 & 0.307938 \\
\hline$-2.60501 e-17$ & 0.307938 & 0.692062 & 0.692062 & 0.307938 \\
\hline$-2.16345 e-17$ & 0.307938 & 0.692062 & 0.692062 & 0.307938 \\
\hline$-1.72189 \mathrm{e}-17$ & 0.307938 & 0.692062 & 0.692062 & 0.307938 \\
\hline$-1.28033 e-17$ & 0.307938 & 0.692062 & 0.692062 & 0.307938 \\
\hline$-8.3877 e-18$ & 0.307938 & 0.692062 & 0.692062 & 0.307938 \\
\hline$-3.9721 e-18$ & 0.307938 & 0.692062 & 0.692062 & 0.307938 \\
\hline$-2.6228 e-18$ & 0.307938 & 0.692062 & 0.692062 & 0.307938 \\
\hline$-2.60916 e-18$ & 0.307938 & 0.692062 & 0.692062 & 0.307938 \\
\hline$-2.59551 e-18$ & 0.307938 & 0.692062 & 0.692062 & 0.307938 \\
\hline$-2.58186 e-18$ & 0.307938 & 0.692062 & 0.692062 & 0.307938 \\
\hline$-2.56822 e-18$ & 0.307938 & 0.692062 & 0.692062 & 0.307938 \\
\hline$-2.55457 e-18$ & 0.307938 & 0.692062 & 0.692062 & 0.307938 \\
\hline$-2.54093 e-18$ & 0.307938 & 0.692062 & 0.692062 & 0.307938 \\
\hline$-2.52728 e-18$ & 0.307938 & 0.692062 & 0.692062 & 0.307938 \\
\hline$-2.51363 e-18$ & 0.307938 & 0.692062 & 0.692062 & 0.307938 \\
\hline$-2.49999 e-18$ & 0.307938 & 0.692062 & 0.692062 & 0.307938 \\
\hline$-2.48634 \mathrm{e}-18$ & 0.307938 & 0.692062 & 0.692062 & 0.307938 \\
\hline$-2.4727 e-18$ & 0.307938 & 0.692062 & 0.692062 & 0.307938 \\
\hline$-2.45905 e-18$ & 0.307938 & 0.692062 & 0.692062 & 0.307938 \\
\hline$-2.4454 e-18$ & 0.307938 & 0.692062 & 0.692062 & 0.307938 \\
\hline$-2.43176 e-18$ & 0.307938 & 0.692062 & 0.692062 & 0.307938 \\
\hline$-2.41811 e-18$ & 0.307938 & 0.692062 & 0.692062 & 0.307938 \\
\hline$-2.40446 e-18$ & 0.307938 & 0.692062 & 0.692062 & 0.307938 \\
\hline$-2.39082 e-18$ & 0.307938 & 0.692062 & 0.692062 & 0.307938 \\
\hline$-2.37717 e-18$ & 0.307938 & 0.692062 & 0.692062 & 0.307938 \\
\hline$-2.36353 e-18$ & 0.307938 & 0.692062 & 0.692062 & 0.307938 \\
\hline$-2.34988 e-18$ & 0.307938 & 0.692062 & 0.692062 & 0.307938 \\
\hline$-2.33623 e-18$ & 0.307938 & 0.692062 & 0.692062 & 0.307938 \\
\hline$-2.32259 e-18$ & 0.307938 & 0.692062 & 0.692062 & 0.307938 \\
\hline$-2.30894 e-18$ & 0.307938 & 0.692062 & 0.692062 & 0.307938 \\
\hline$-2.2953 e-18$ & 0.307938 & 0.692062 & 0.692062 & 0.307938 \\
\hline$-2.28165 e-18$ & 0.307938 & 0.692062 & 0.692062 & 0.307938 \\
\hline$-2.268 e-18$ & 0.307938 & 0.692062 & 0.692062 & 0.307938 \\
\hline$-2.25436 e-18$ & 0.307938 & 0.692062 & 0.692062 & 0.307938 \\
\hline$-2.24071 e-18$ & 0.307938 & 0.692062 & 0.692062 & 0.307938 \\
\hline$-2.22706 e-18$ & 0.307938 & 0.692062 & 0.692062 & 0.307938 \\
\hline$-2.21342 e-18$ & 0.307938 & 0.692062 & 0.692062 & 0.307938 \\
\hline
\end{tabular}




$\begin{array}{llllll}94 & -2.19977 \mathrm{e}-18 & 0.307938 & 0.692062 & 0.692062 & 0.307938 \\ 95 & -2.18613 \mathrm{e}-18 & 0.307938 & 0.692062 & 0.692062 & 0.307938 \\ 96 & -2.17248 \mathrm{e}-18 & 0.307938 & 0.692062 & 0.692062 & 0.307938 \\ 97 & -2.15883 \mathrm{e}-18 & 0.307938 & 0.692062 & 0.692062 & 0.307938 \\ 98 & -2.14519 \mathrm{e}-18 & 0.307938 & 0.692062 & 0.692062 & 0.307938 \\ 99 & -2.13154 \mathrm{e}-18 & 0.307938 & 0.692062 & 0.692062 & 0.307938 \\ 100 & -2.1179 \mathrm{e}-18 & 0.307938 & 0.692062 & 0.692062 & 0.307938\end{array}$

Fitted time course for 4-COOMe aryl bromide and 4- $\mathrm{H}$ aryl bromide

Input: k1 (reaction 1): 5.6; k1 (reaction 2): 1

$\begin{array}{llllll}\text { \# Time Fe } & \text { Ar1 } & \text { Ar2 } & \text { Fe1 } & \text { Fe2 } \\ 0 & 1 & 1 & 1 & 0 & 0 \\ 1 & 0.0459273 & 0.259825 & 0.786102 & 0.740175 & 0.213898 \\ 2 & 0.00543763 & 0.233934 & 0.771504 & 0.766066 & 0.228496 \\ 3 & 0.000686634 & 0.23095 & 0.769737 & 0.76905 & 0.230263 \\ 4 & 8.73817 \mathrm{e}-05 & 0.230574 & 0.769513 & 0.769426 & 0.230487 \\ 5 & 1.11312 \mathrm{e}-05 & 0.230526 & 0.769485 & 0.769474 & 0.230515 \\ 6 & 1.41814 \mathrm{e}-06 & 0.23052 & 0.769481 & 0.76948 & 0.230519 \\ 7 & 1.80677 \mathrm{e}-07 & 0.230519 & 0.769481 & 0.769481 & 0.230519 \\ 8 & 2.3019 \mathrm{e}-08 & 0.230519 & 0.769481 & 0.769481 & 0.230519 \\ 9 & 2.93279 \mathrm{e}-09 & 0.230519 & 0.769481 & 0.769481 & 0.230519 \\ 10 & 3.73643 \mathrm{e}-10 & 0.230519 & 0.769481 & 0.769481 & 0.230519 \\ 11 & 4.75622 \mathrm{e}-11 & 0.230519 & 0.769481 & 0.769481 & 0.230519 \\ 12 & 6.11878 \mathrm{e}-12 & 0.230519 & 0.769481 & 0.769481 & 0.230519 \\ 13 & 7.49338 \mathrm{e}-13 & 0.230519 & 0.769481 & 0.769481 & 0.230519 \\ 14 & 1.05084 \mathrm{e}-13 & 0.230519 & 0.769481 & 0.769481 & 0.230519 \\ 15 & 2.08496 \mathrm{e}-15 & 0.230519 & 0.769481 & 0.769481 & 0.230519 \\ 16 & -2.86031 \mathrm{e}-14 & 0.230519 & 0.769481 & 0.769481 & 0.230519 \\ 17 & -2.21289 \mathrm{e}-14 & 0.230519 & 0.769481 & 0.769481 & 0.230519 \\ 18 & -1.18613 \mathrm{e}-14 & 0.230519 & 0.769481 & 0.769481 & 0.230519 \\ 19 & -5.67801 \mathrm{e}-15 & 0.230519 & 0.769481 & 0.769481 & 0.230519 \\ 20 & -2.18167 \mathrm{e}-15 & 0.230519 & 0.769481 & 0.769481 & 0.230519 \\ 21 & -2.05391 \mathrm{e}-15 & 0.230519 & 0.769481 & 0.769481 & 0.230519 \\ 22 & -1.92616 \mathrm{e}-15 & 0.230519 & 0.769481 & 0.769481 & 0.230519 \\ 23 & -1.7984 \mathrm{e}-15 & 0.230519 & 0.769481 & 0.769481 & 0.230519 \\ 24 & -1.67065 \mathrm{e}-15 & 0.230519 & 0.769481 & 0.769481 & 0.230519 \\ 25 & -1.54289 \mathrm{e}-15 & 0.230519 & 0.769481 & 0.769481 & 0.230519 \\ 26 & -1.41514 \mathrm{e}-15 & 0.230519 & 0.769481 & 0.769481 & 0.230519 \\ 27 & -1.28738 \mathrm{e}-15 & 0.230519 & 0.769481 & 0.769481 & 0.230519 \\ 28 & -1.15963 \mathrm{e}-15 & 0.230519 & 0.769481 & 0.769481 & 0.230519 \\ 29 & -1.03187 \mathrm{e}-15 & 0.230519 & 0.769481 & 0.769481 & 0.230519 \\ 30 & -9.04117 \mathrm{e}-16 & 0.230519 & 0.769481 & 0.769481 & 0.230519\end{array}$




\begin{tabular}{|c|c|c|c|c|c|}
\hline 31 & $-7.76362 \mathrm{e}-16$ & 0.230519 & 0.769481 & 0.769481 & 0.230519 \\
\hline 32 & $-6.48607 e-16$ & 0.230519 & 0.769481 & 0.769481 & 0.230519 \\
\hline 33 & $-5.20852 e-16$ & 0.230519 & 0.769481 & 0.769481 & 0.230519 \\
\hline 34 & $-3.93097 e-16$ & 0.230519 & 0.769481 & 0.769481 & 0.230519 \\
\hline 35 & $-2.65342 e-16$ & 0.230519 & 0.769481 & 0.769481 & 0.230519 \\
\hline 36 & $-1.37587 e-16$ & 0.230519 & 0.769481 & 0.769481 & 0.230519 \\
\hline 37 & $-6.0543 e-17$ & 0.230519 & 0.769481 & 0.769481 & 0.230519 \\
\hline 38 & $-5.69624 e-17$ & 0.230519 & 0.769481 & 0.769481 & 0.230519 \\
\hline 39 & $-5.33817 e-17$ & 0.230519 & 0.769481 & 0.769481 & 0.230519 \\
\hline 40 & $-4.9801 e-17$ & 0.230519 & 0.769481 & 0.769481 & 0.230519 \\
\hline 41 & $-4.62203 e-17$ & 0.230519 & 0.769481 & 0.769481 & 0.230519 \\
\hline 42 & $-4.26396 e-17$ & 0.230519 & 0.769481 & 0.769481 & 0.230519 \\
\hline 43 & $-3.90589 e-17$ & 0.230519 & 0.769481 & 0.769481 & 0.230519 \\
\hline 44 & $-3.54783 e-17$ & 0.230519 & 0.769481 & 0.769481 & 0.230519 \\
\hline 45 & $-3.18976 e-17$ & 0.230519 & 0.769481 & 0.769481 & 0.230519 \\
\hline 46 & $-2.83169 e-17$ & 0.230519 & 0.769481 & 0.769481 & 0.230519 \\
\hline 47 & $-2.47362 e-17$ & 0.230519 & 0.769481 & 0.769481 & 0.230519 \\
\hline 48 & $-2.11555 e-17$ & 0.230519 & 0.769481 & 0.769481 & 0.230519 \\
\hline 49 & $-1.75748 e-17$ & 0.230519 & 0.769481 & 0.769481 & 0.230519 \\
\hline 50 & $-1.39942 e-17$ & 0.230519 & 0.769481 & 0.769481 & 0.230519 \\
\hline 51 & $-1.04135 e-17$ & 0.230519 & 0.769481 & 0.769481 & 0.230519 \\
\hline 52 & $-6.83279 e-18$ & 0.230519 & 0.769481 & 0.769481 & 0.230519 \\
\hline 53 & $-3.2521 e-18$ & 0.230519 & 0.769481 & 0.769481 & 0.230519 \\
\hline 54 & $-1.73193 e-18$ & 0.230519 & 0.769481 & 0.769481 & 0.230519 \\
\hline 55 & $-1.72163 e-18$ & 0.230519 & 0.769481 & 0.769481 & 0.230519 \\
\hline 56 & $-1.71134 e-18$ & 0.230519 & 0.769481 & 0.769481 & 0.230519 \\
\hline 57 & $-1.70104 e-18$ & 0.230519 & 0.769481 & 0.769481 & 0.230519 \\
\hline 58 & $-1.69074 e-18$ & 0.230519 & 0.769481 & 0.769481 & 0.230519 \\
\hline 59 & $-1.68045 e-18$ & 0.230519 & 0.769481 & 0.769481 & 0.230519 \\
\hline 60 & $-1.67015 e-18$ & 0.230519 & 0.769481 & 0.769481 & 0.230519 \\
\hline 61 & $-1.65986 e-18$ & 0.230519 & 0.769481 & 0.769481 & 0.230519 \\
\hline 62 & $-1.64956 e-18$ & 0.230519 & 0.769481 & 0.769481 & 0.230519 \\
\hline 63 & $-1.63927 e-18$ & 0.230519 & 0.769481 & 0.769481 & 0.230519 \\
\hline 64 & $-1.62897 e-18$ & 0.230519 & 0.769481 & 0.769481 & 0.230519 \\
\hline 65 & $-1.61868 e-18$ & 0.230519 & 0.769481 & 0.769481 & 0.230519 \\
\hline 66 & $-1.60838 e-18$ & 0.230519 & 0.769481 & 0.769481 & 0.230519 \\
\hline 67 & $-1.59808 e-18$ & 0.230519 & 0.769481 & 0.769481 & 0.230519 \\
\hline 68 & $-1.58779 e-18$ & 0.230519 & 0.769481 & 0.769481 & 0.230519 \\
\hline 69 & $-1.57749 e-18$ & 0.230519 & 0.769481 & 0.769481 & 0.230519 \\
\hline 70 & $-1.5672 e-18$ & 0.230519 & 0.769481 & 0.769481 & 0.230519 \\
\hline 71 & $-1.5569 e-18$ & 0.230519 & 0.769481 & 0.769481 & 0.230519 \\
\hline 72 & $-1.54661 e-18$ & 0.230519 & 0.769481 & 0.769481 & 0.230519 \\
\hline 73 & $-1.53631 e-18$ & 0.230519 & 0.769481 & 0.769481 & 0.230519 \\
\hline 74 & $-1.52602 e-18$ & 0.230519 & 0.769481 & 0.769481 & 0.230519 \\
\hline
\end{tabular}




$\begin{array}{llllll}75 & -1.51572 \mathrm{e}-18 & 0.230519 & 0.769481 & 0.769481 & 0.230519 \\ 76 & -1.50542 \mathrm{e}-18 & 0.230519 & 0.769481 & 0.769481 & 0.230519 \\ 77 & -1.49513 \mathrm{e}-18 & 0.230519 & 0.769481 & 0.769481 & 0.230519 \\ 78 & -1.48483 \mathrm{e}-18 & 0.230519 & 0.769481 & 0.769481 & 0.230519 \\ 79 & -1.47454 \mathrm{e}-18 & 0.230519 & 0.769481 & 0.769481 & 0.230519 \\ 80 & -1.46424 \mathrm{e}-18 & 0.230519 & 0.769481 & 0.769481 & 0.230519 \\ 81 & -1.45395 \mathrm{e}-18 & 0.230519 & 0.769481 & 0.769481 & 0.230519 \\ 82 & -1.44365 \mathrm{e}-18 & 0.230519 & 0.769481 & 0.769481 & 0.230519 \\ 83 & -1.43336 \mathrm{e}-18 & 0.230519 & 0.769481 & 0.769481 & 0.230519 \\ 84 & -1.42306 \mathrm{e}-18 & 0.230519 & 0.769481 & 0.769481 & 0.230519 \\ 85 & -1.41276 \mathrm{e}-18 & 0.230519 & 0.769481 & 0.769481 & 0.230519 \\ 86 & -1.40247 \mathrm{e}-18 & 0.230519 & 0.769481 & 0.769481 & 0.230519 \\ 87 & -1.39217 \mathrm{e}-18 & 0.230519 & 0.769481 & 0.769481 & 0.230519 \\ 88 & -1.38188 \mathrm{e}-18 & 0.230519 & 0.769481 & 0.769481 & 0.230519 \\ 89 & -1.37158 \mathrm{e}-18 & 0.230519 & 0.769481 & 0.769481 & 0.230519 \\ 90 & -1.36129 \mathrm{e}-18 & 0.230519 & 0.769481 & 0.769481 & 0.230519 \\ 91 & -1.35099 \mathrm{e}-18 & 0.230519 & 0.769481 & 0.769481 & 0.230519 \\ 92 & -1.3407 \mathrm{e}-18 & 0.230519 & 0.769481 & 0.769481 & 0.230519 \\ 93 & -1.3304 \mathrm{e}-18 & 0.230519 & 0.769481 & 0.769481 & 0.230519 \\ 94 & -1.3201 \mathrm{e}-18 & 0.230519 & 0.769481 & 0.769481 & 0.230519 \\ 95 & -1.30981 \mathrm{e}-18 & 0.230519 & 0.769481 & 0.769481 & 0.230519 \\ 96 & -1.29951 \mathrm{e}-18 & 0.230519 & 0.769481 & 0.769481 & 0.230519 \\ 97 & -1.28922 \mathrm{e}-18 & 0.230519 & 0.769481 & 0.769481 & 0.230519 \\ 98 & -1.27892 \mathrm{e}-18 & 0.230519 & 0.769481 & 0.769481 & 0.230519 \\ 99 & -1.26863 \mathrm{e}-18 & 0.230519 & 0.769481 & 0.769481 & 0.230519 \\ 100 & -1.25833 \mathrm{e}-18 & 0.230519 & 0.769481 & 0.769481 & 0.230519\end{array}$




\section{Additional Spectroscopic Data}
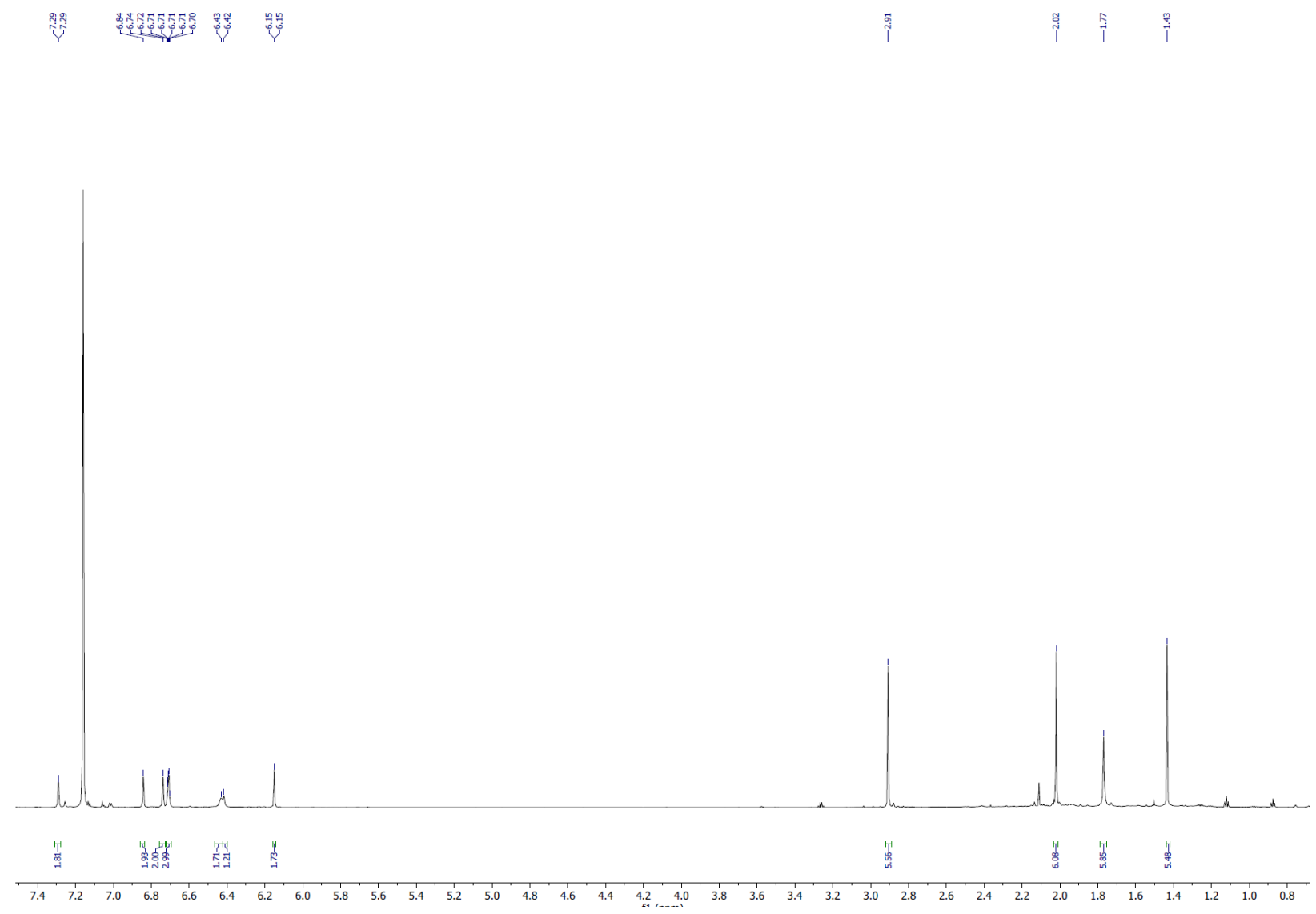

Figure S5. ${ }^{1} \mathrm{H}$ NMR spectrum of $\left(3,5-\mathrm{Me}_{2}{ }^{\mathrm{Mes}} \mathrm{CNC}\right) \mathrm{FePh}\left(\mathrm{N}_{2}\right) \mathrm{Br}$ in $\mathrm{C}_{6} \mathrm{D}_{6}$ at $25^{\circ} \mathrm{C}$. 

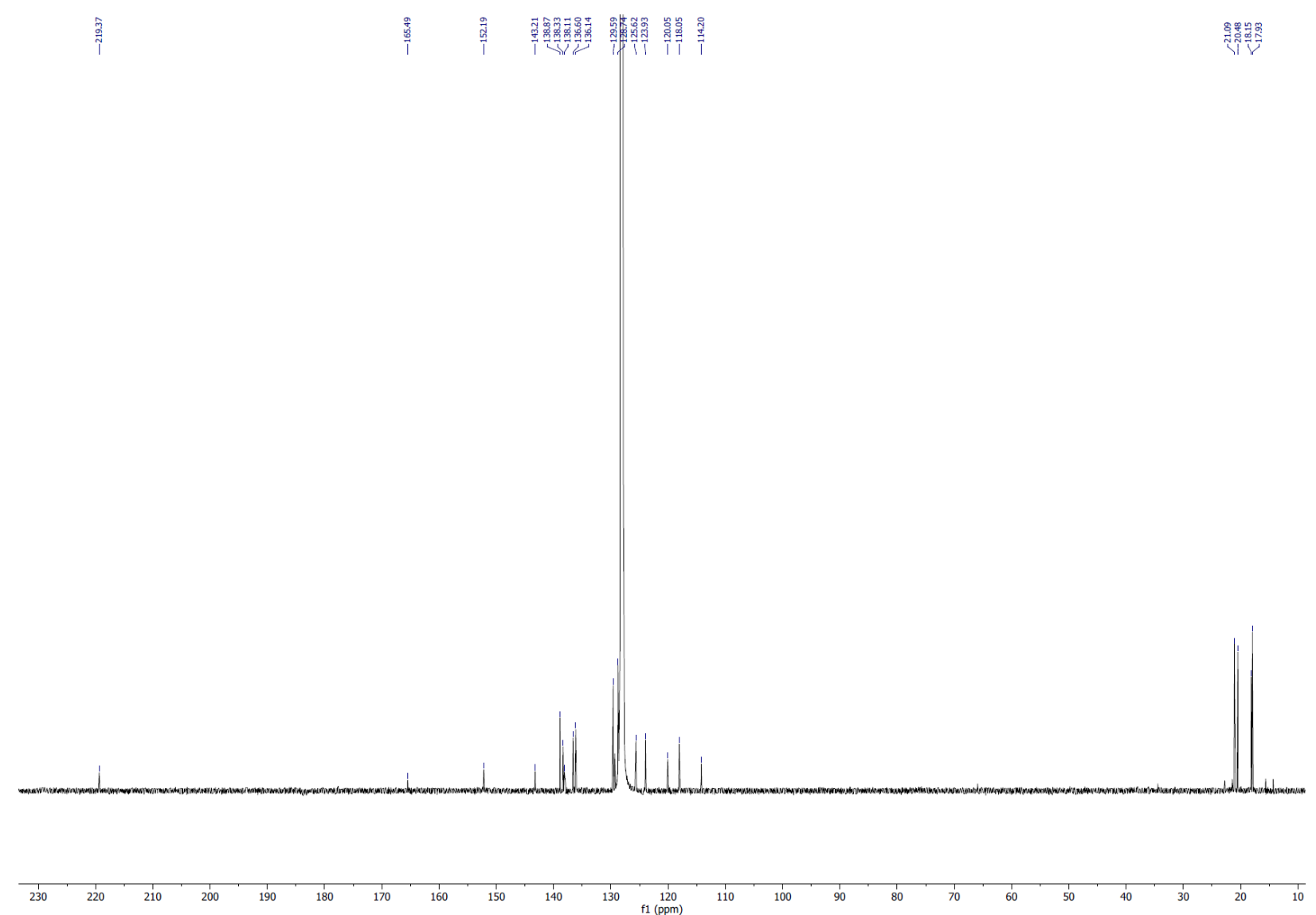

Figure S6. ${ }^{13} \mathrm{C}-\mathrm{NMR}$ spectrum of $\left(3,5-\mathrm{Me}_{2}{ }^{\mathrm{Mes}} \mathrm{CNC}\right) \mathrm{FePh}\left(\mathrm{N}_{2}\right) \mathrm{Br}$ in $\mathrm{C}_{6} \mathrm{D}_{6}$ at $25^{\circ} \mathrm{C}$. 


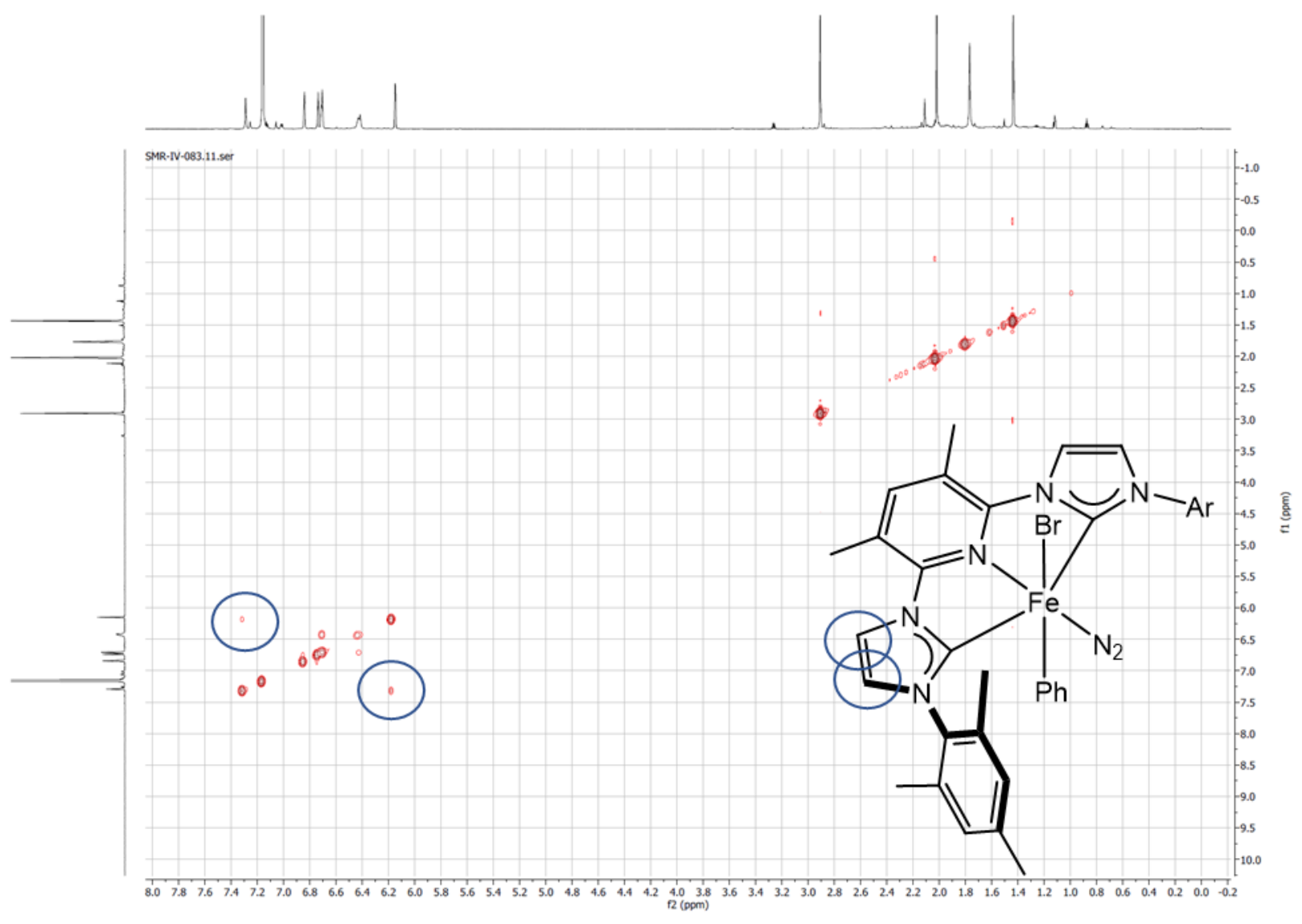

Figure S7. COSY spectrum of $\left(3,5-\mathrm{Me}_{2}{ }^{\mathrm{Mes}} \mathrm{CNC}\right) \mathrm{FePh}\left(\mathrm{N}_{2}\right) \mathrm{Br}$ in $\mathrm{C}_{6} \mathrm{D}_{6}$ at $25^{\circ} \mathrm{C}$. 


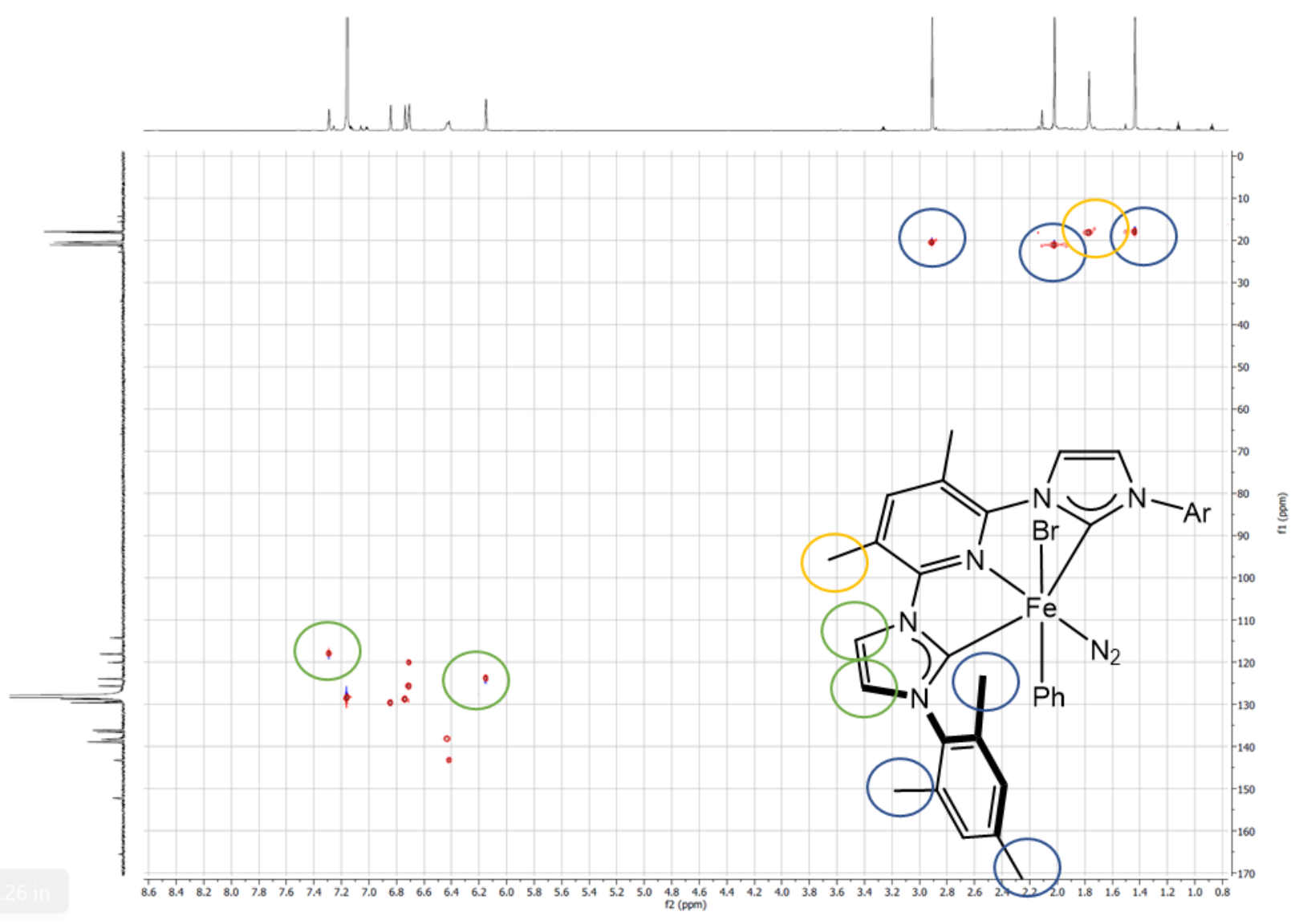

Figure S8. $\mathrm{HSQC}$ spectrum of $\left(3,5-\mathrm{Me}_{2}{ }^{\mathrm{Mes}} \mathrm{CNC}\right) \mathrm{FePh}\left(\mathrm{N}_{2}\right) \mathrm{Br}$ in $\mathrm{C}_{6} \mathrm{D}_{6}$ at $25^{\circ} \mathrm{C}$. 


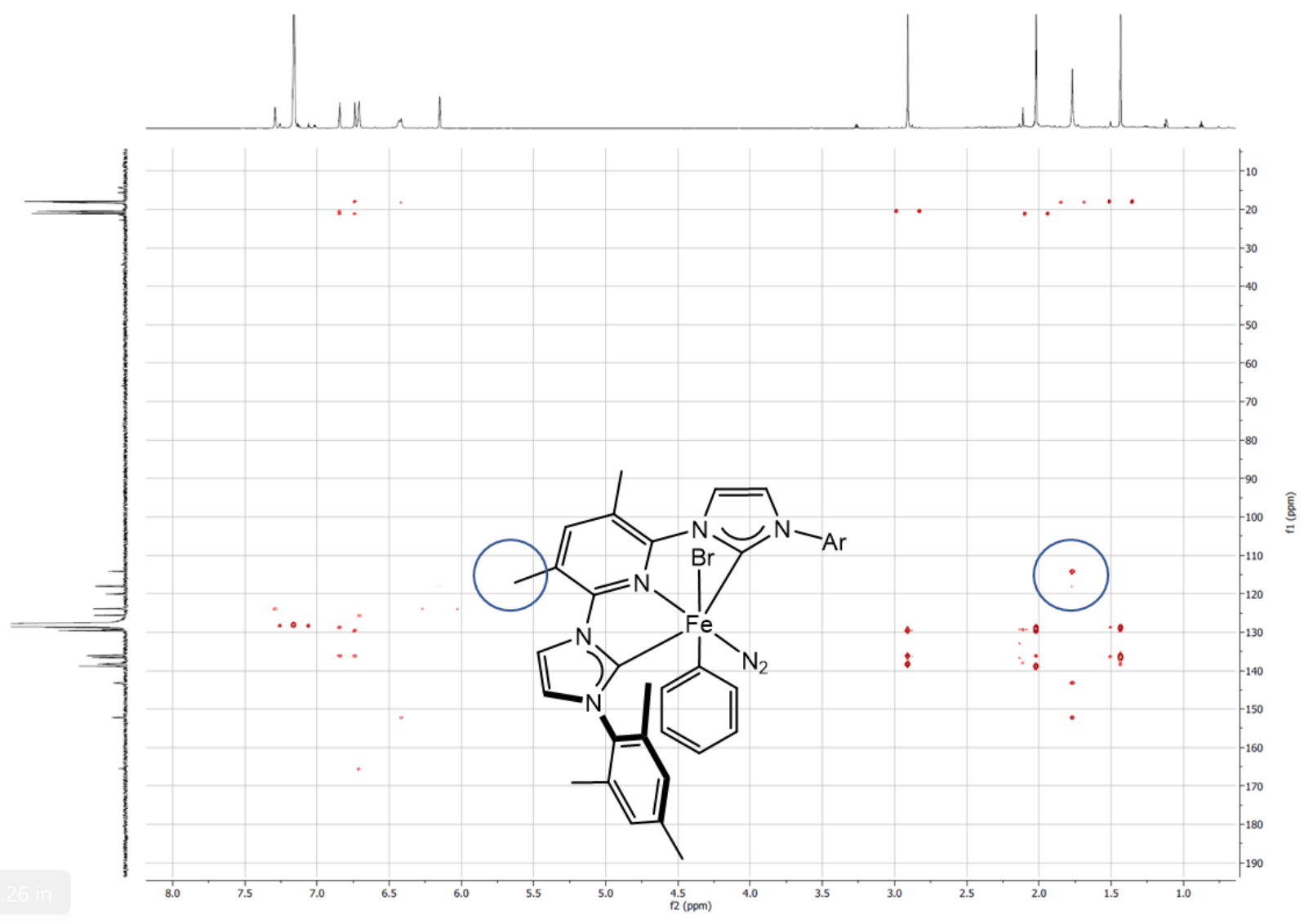

Figure S9. $\mathrm{HMBC}$ spectrum of $\left(3,5-\mathrm{Me}_{2}{ }^{\mathrm{Mes}} \mathrm{CNC}\right) \mathrm{FePh}\left(\mathrm{N}_{2}\right) \mathrm{Br}$ in $\mathrm{C}_{6} \mathrm{D}_{6}$ at $25^{\circ} \mathrm{C}$. 


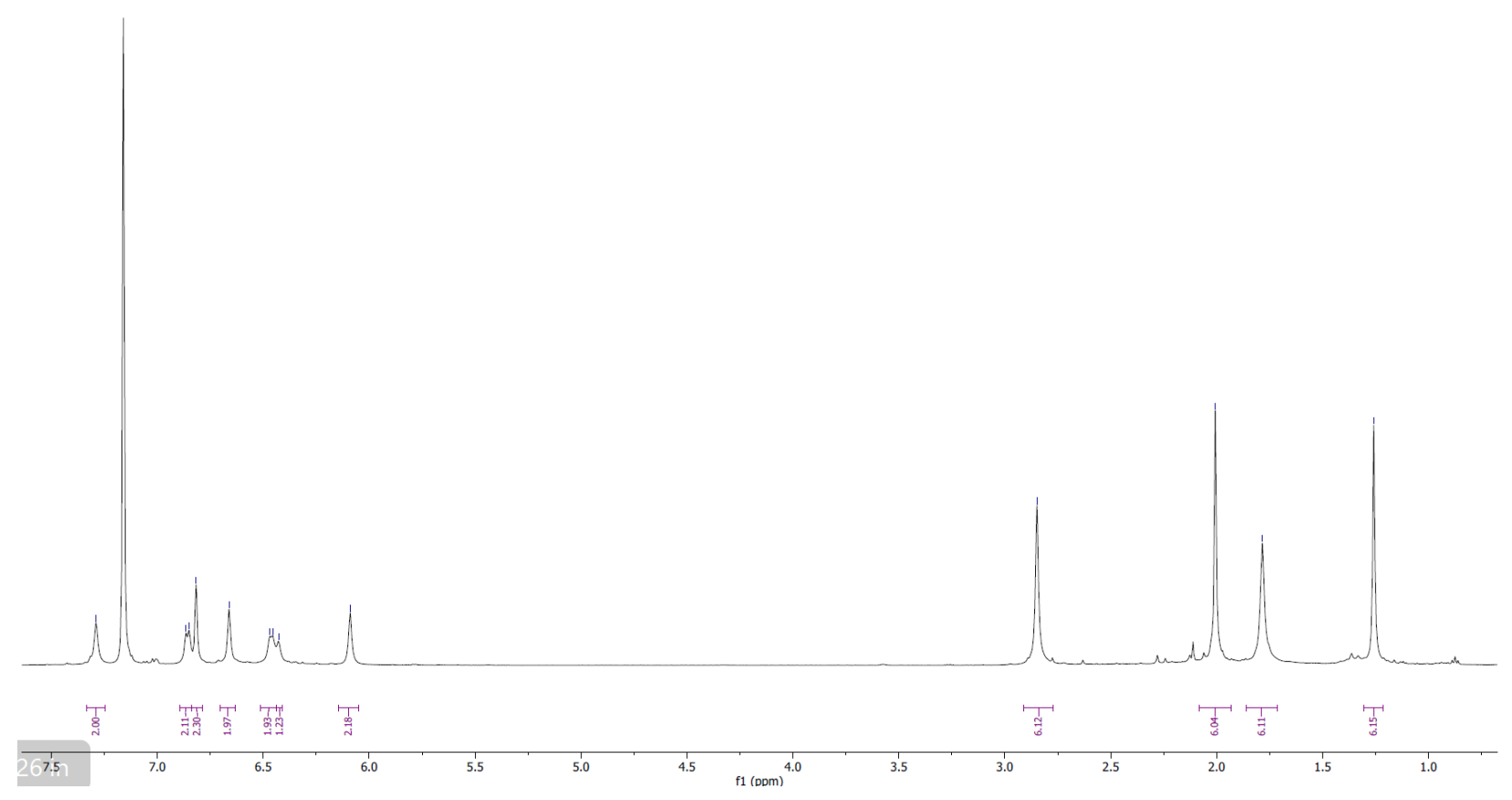

Figure S10. ${ }^{1} \mathrm{H}-\mathrm{NMR}$ spectrum of $\left(3,5-\mathrm{Me}_{2}{ }^{\mathrm{Mes}} \mathrm{CNC}\right) \mathrm{Fe}\left(\mathrm{C}_{6} \mathrm{H}_{4} \mathrm{CF}_{3}\right)\left(\mathrm{N}_{2}\right) \mathrm{Br}$ in $\mathrm{C}_{6} \mathrm{D}_{6}$ at 25 ${ }^{\circ} \mathrm{C}$. 


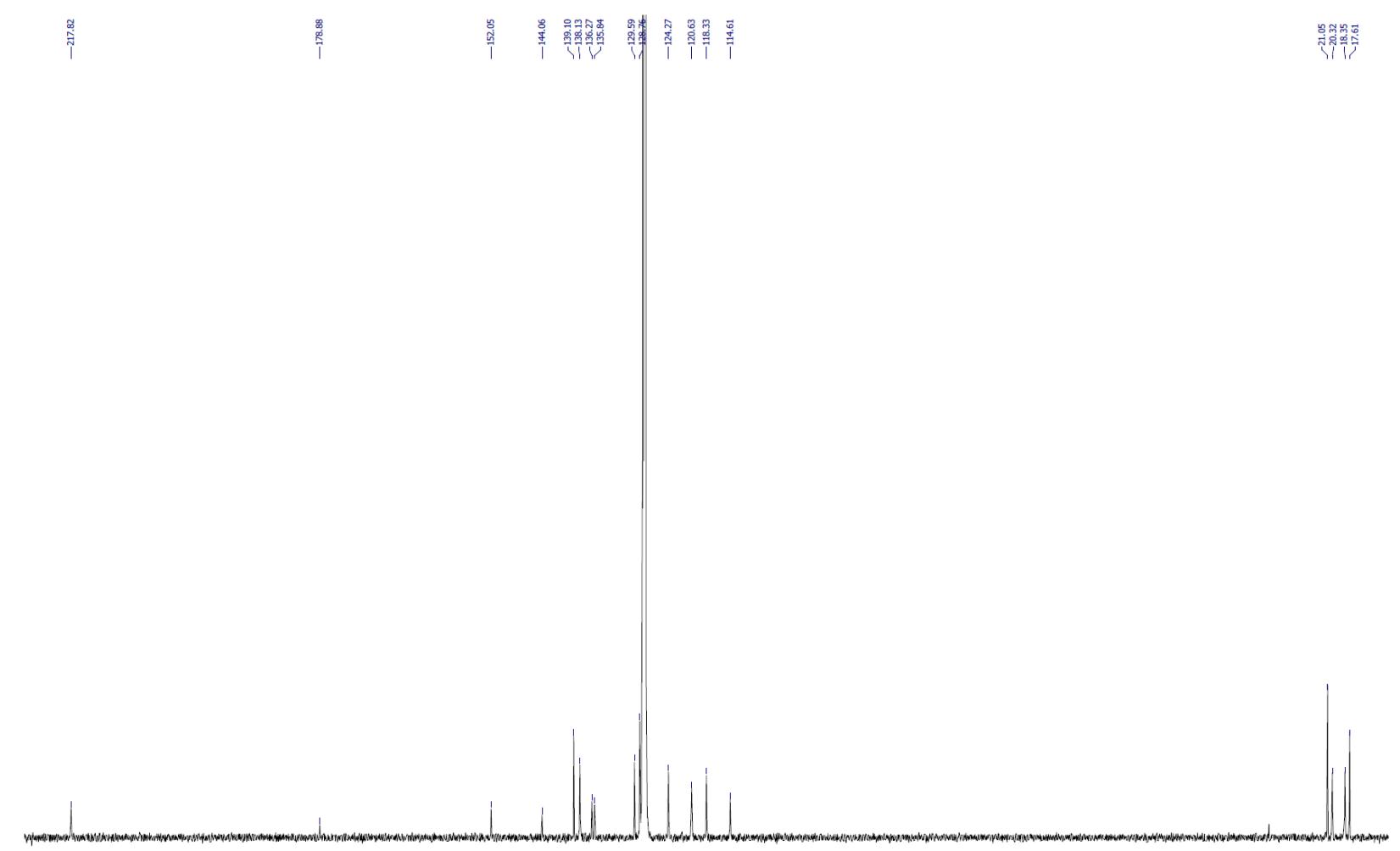

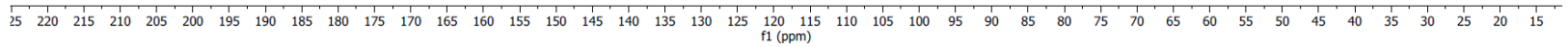

Figure S11. ${ }^{13} \mathrm{C}-\mathrm{NMR}$ spectrum of $\left(3,5-\mathrm{Me}_{2}{ }^{\mathrm{Mes}} \mathrm{CNC}\right) \mathrm{Fe}\left(\mathrm{C}_{6} \mathrm{H}_{4} \mathrm{CF}_{3}\right)\left(\mathrm{N}_{2}\right) \mathrm{Br}$ in $\mathrm{C}_{6} \mathrm{D}_{6}$ at 25 ${ }^{\circ} \mathrm{C}$. 


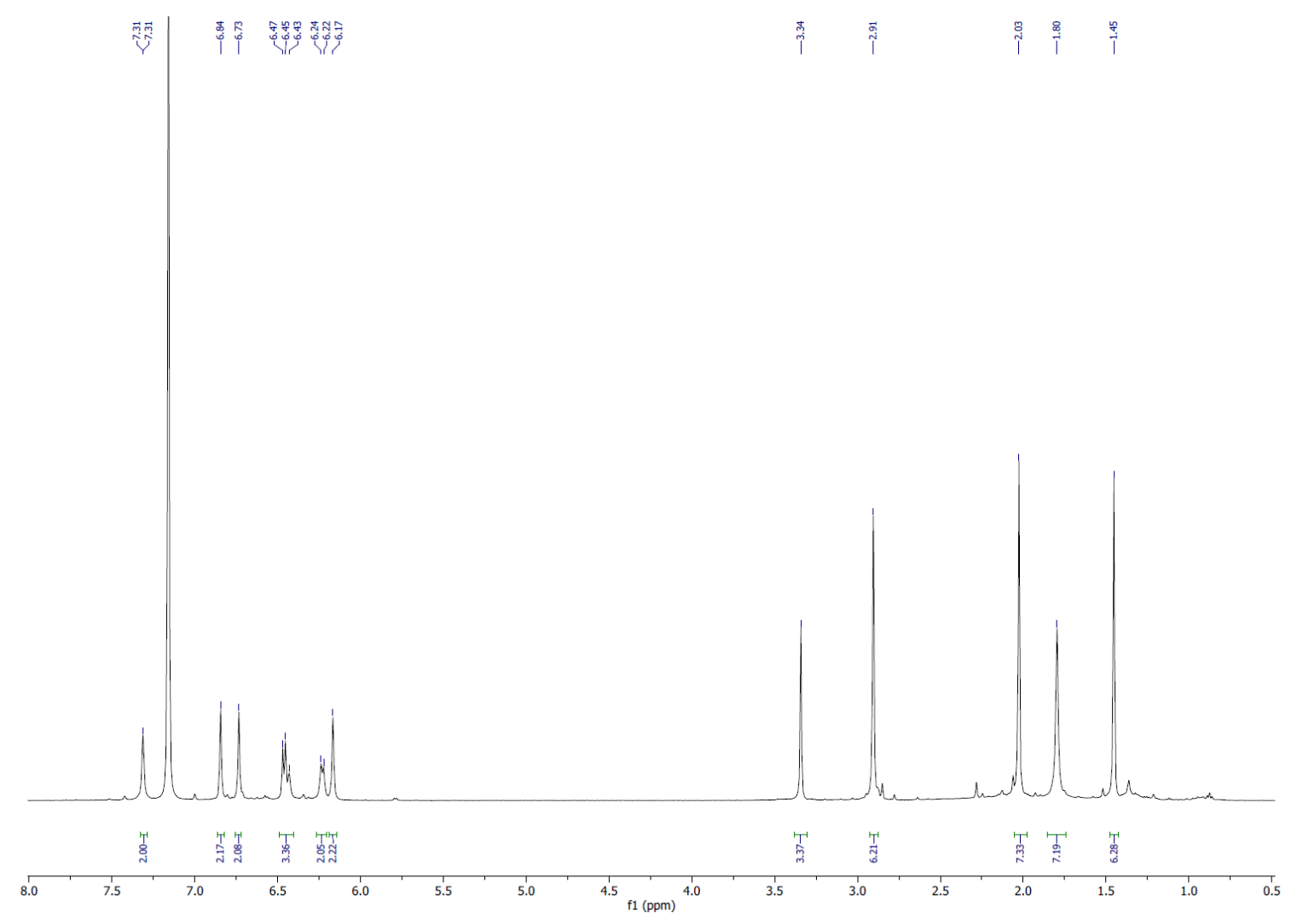

Figure S12. ${ }^{1} \mathrm{H}-\mathrm{NMR}$ spectrum of $\left(3,5-\mathrm{Me}_{2}{ }^{\mathrm{Mes}} \mathrm{CNC}\right) \mathrm{Fe}\left(\mathrm{C}_{6} \mathrm{H}_{4} \mathrm{OMe}\right)\left(\mathrm{N}_{2}\right) \mathrm{Br}$ in $\mathrm{C}_{6} \mathrm{D}_{6}$ at 25 ${ }^{\circ} \mathrm{C}$. 


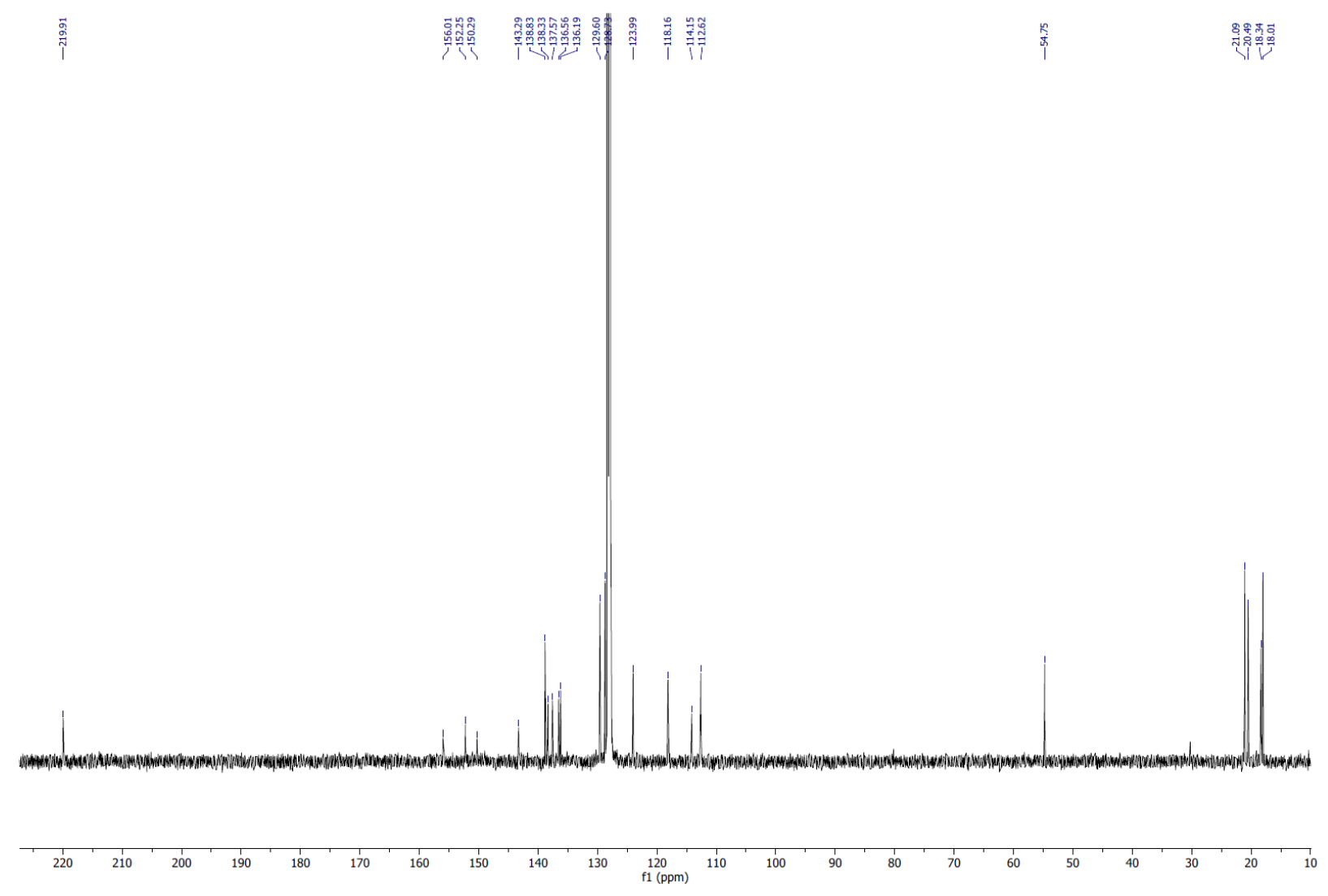

Figure S13. ${ }^{13} \mathrm{C}-\mathrm{NMR}$ spectrum of $\left(3,5-\mathrm{Me}_{2}-{ }^{\mathrm{Mes}} \mathrm{CNC}\right) \mathrm{Fe}\left(\mathrm{C}_{6} \mathrm{H}_{4} \mathrm{OMe}\right)\left(\mathrm{N}_{2}\right) \mathrm{Br}$ in $\mathrm{C}_{6} \mathrm{D}_{6}$ at 25 ${ }^{\circ} \mathrm{C}$. 


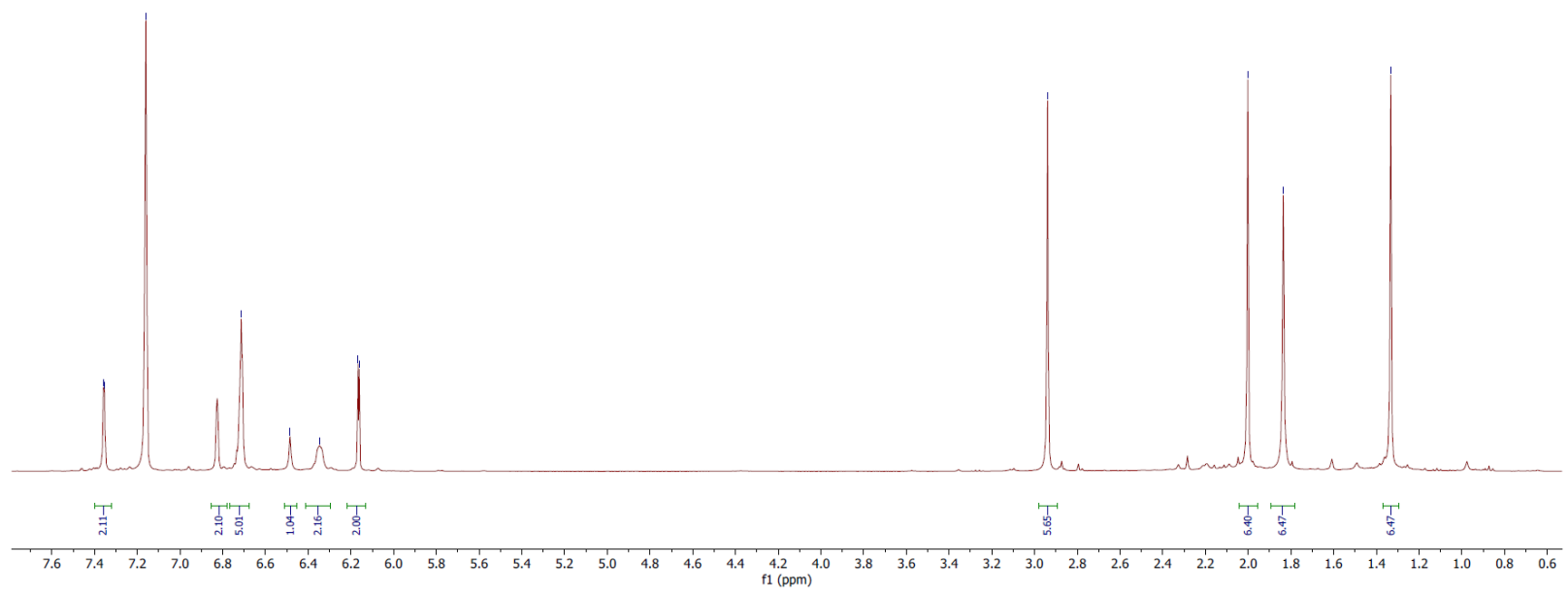

Figure S14. ${ }^{1} \mathrm{H}-\mathrm{NMR}$ spectrum of $\left(3,5-\mathrm{Me}_{2}{ }^{\mathrm{Mes}} \mathrm{CNC}\right) \mathrm{FePh}\left(\mathrm{N}_{2}\right) \mathrm{I}$ in $\mathrm{C}_{6} \mathrm{D}_{6}$ at $25^{\circ} \mathrm{C}$. 


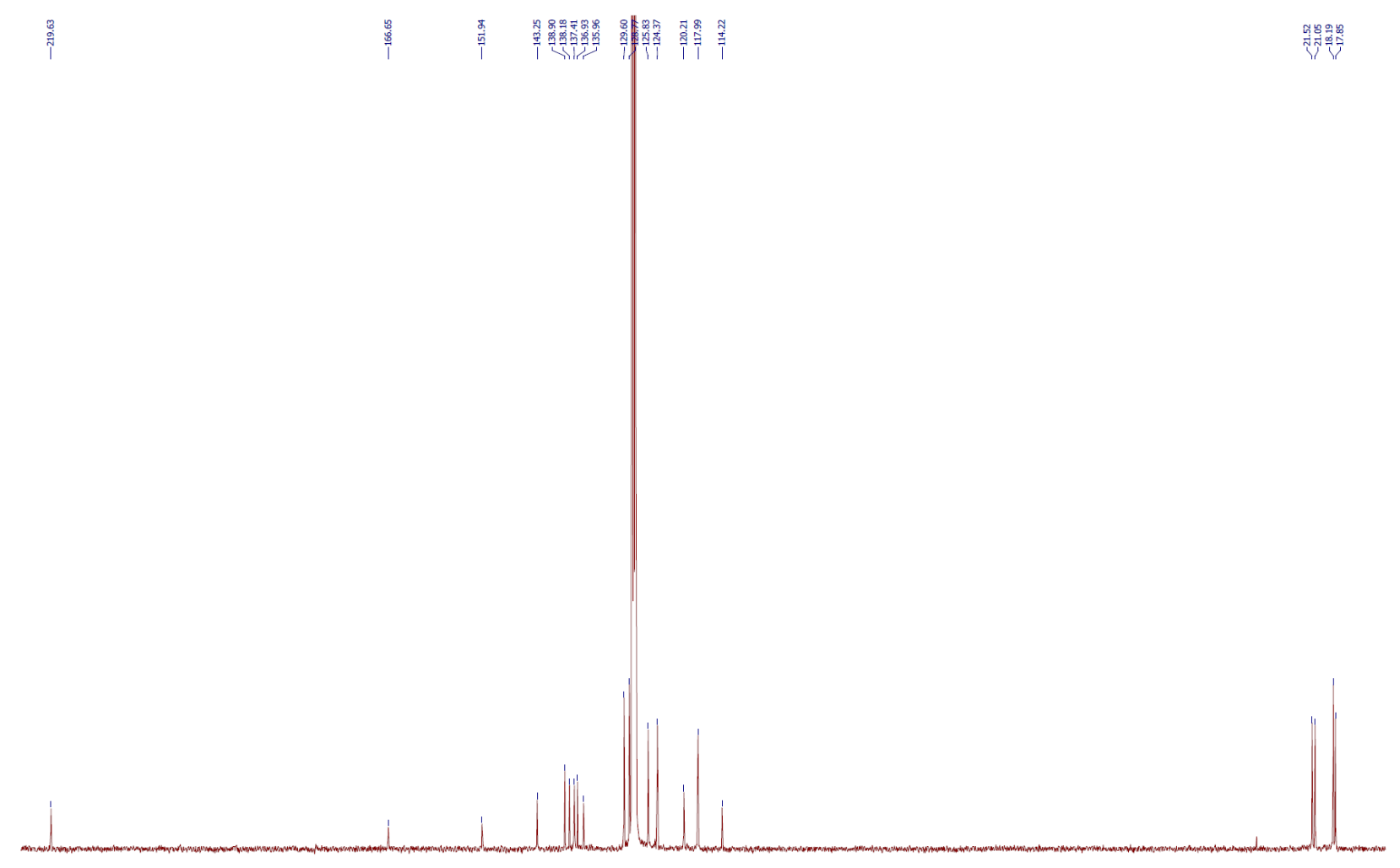

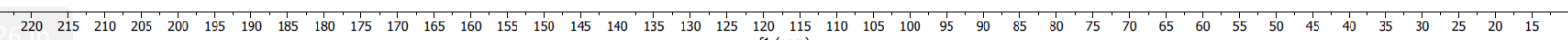

Figure S15. ${ }^{13} \mathrm{C}-\mathrm{NMR}$ spectrum of $\left(3,5-\mathrm{Me}_{2}-{ }^{\mathrm{Mes}} \mathrm{CNC}\right) \mathrm{FePh}\left(\mathrm{N}_{2}\right) \mathrm{I}$ in $\mathrm{C}_{6} \mathrm{D}_{6}$ at $25^{\circ} \mathrm{C}$. 


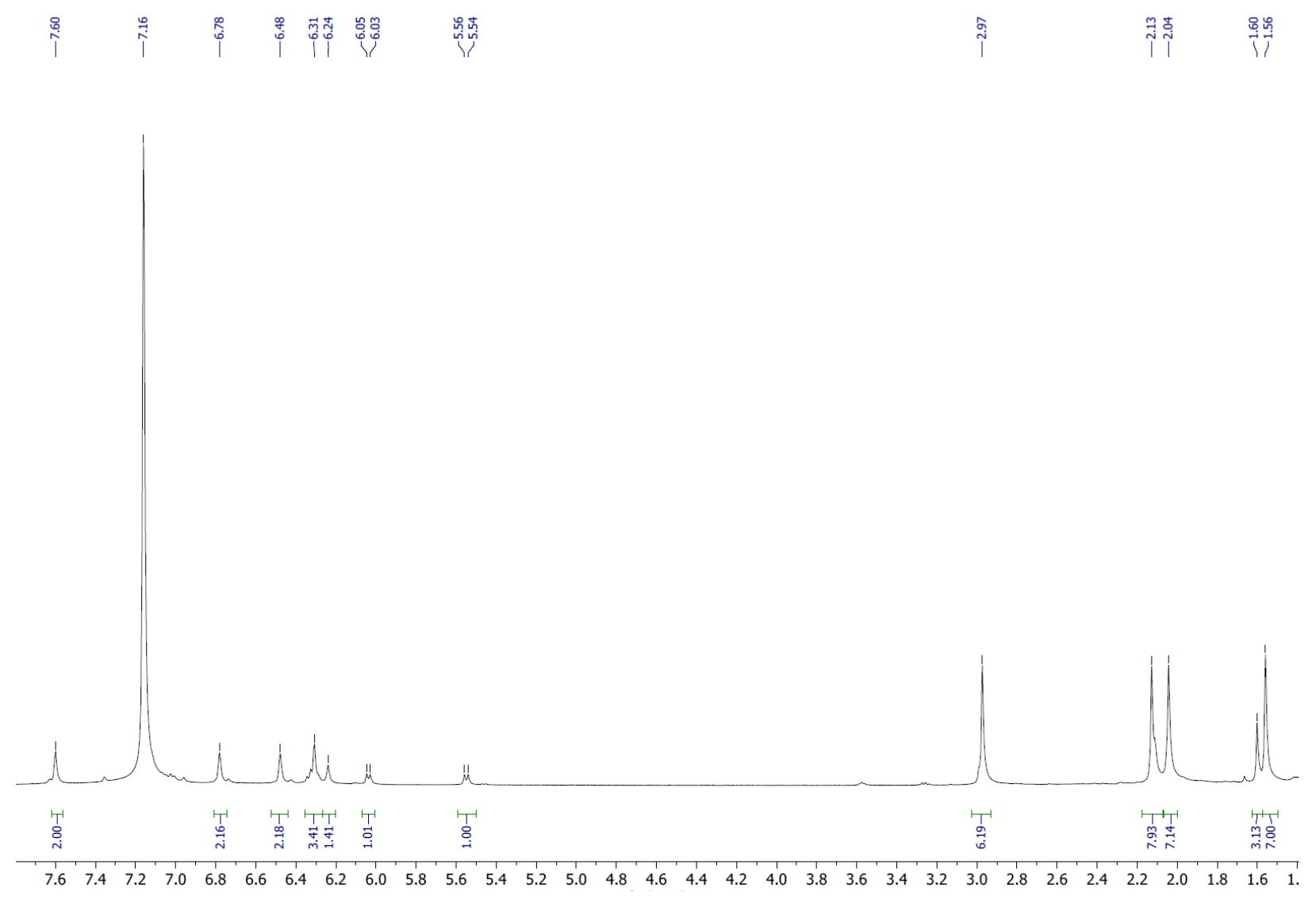

Figure S16. ${ }^{1} \mathrm{H}-\mathrm{NMR}$ spectrum of $\left(3,5-\mathrm{Me}_{2}-{ }^{\mathrm{Mes}} \mathrm{CNC}\right) \mathrm{Fe}\left(6-\mathrm{Me}\right.$ pyridine-2-yl) $\mathrm{Br}$ in $\mathrm{C}_{6} \mathrm{D}_{6}$ at $25^{\circ} \mathrm{C}$. 


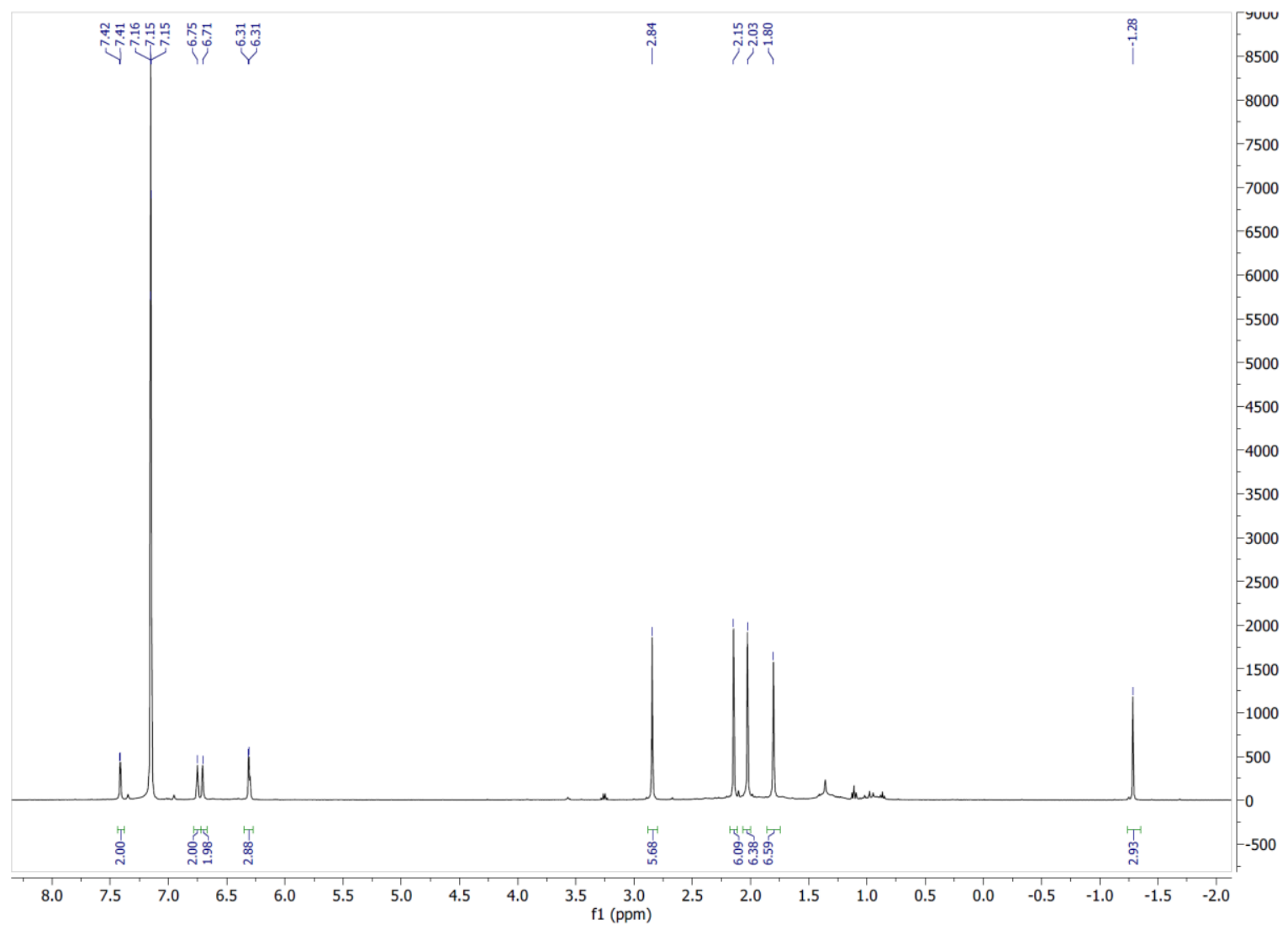

Figure S17. ${ }^{1} \mathrm{H}$ NMR spectrum of $\left(3,5-\mathrm{Me}_{2}-{ }^{\mathrm{Mes}} \mathrm{CNC}\right) \mathrm{Fe}(\mathrm{Me})\left(\mathrm{N}_{2}\right)(\mathrm{I})$ in $\mathrm{C}_{6} \mathrm{D}_{6}$ at $25^{\circ} \mathrm{C}$. 


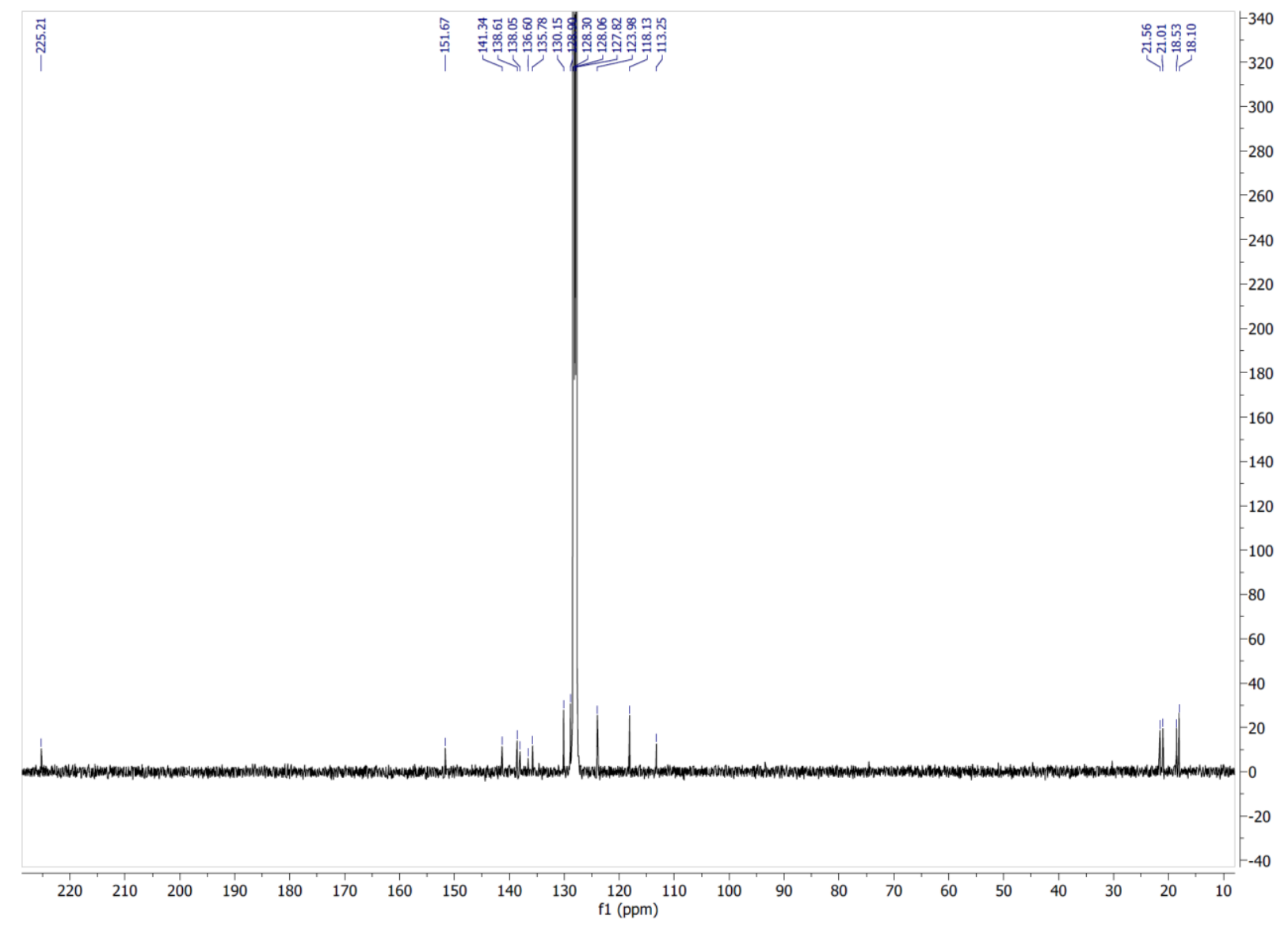

Figure S18. ${ }^{13} \mathrm{C}$ NMR spectrum of $\left(3,5-\mathrm{Me}_{2-}{ }^{\mathrm{Mes}} \mathrm{CNC}\right) \mathrm{Fe}(\mathrm{Me})\left(\mathrm{N}_{2}\right)(\mathrm{I})$ in $\mathrm{C}_{6} \mathrm{D}_{6}$ at $25^{\circ} \mathrm{C}$. 


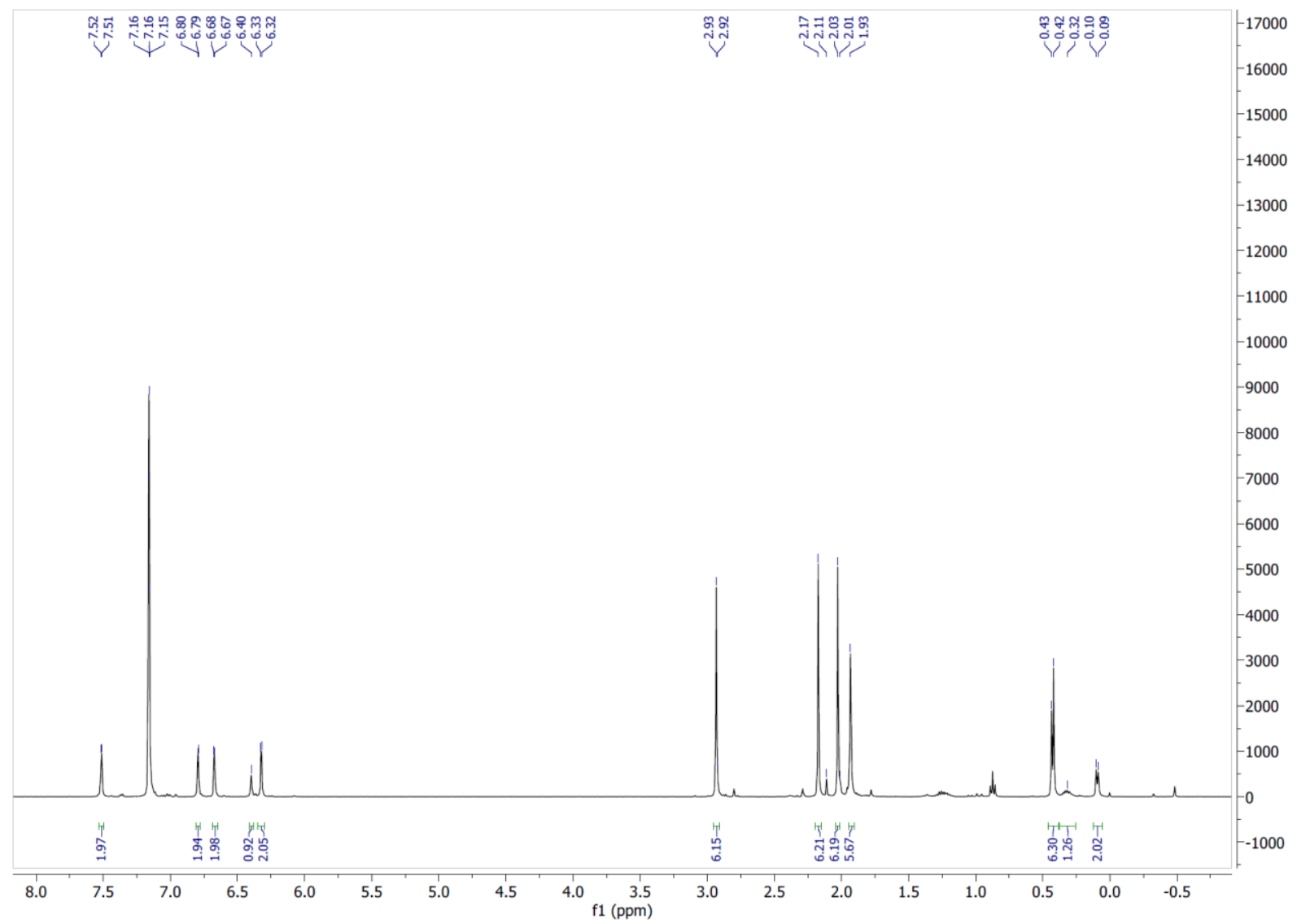

Figure S19. ${ }^{1} \mathrm{H}-\mathrm{NMR}$ spectrum of $\left(3,5-\mathrm{Me}_{2-}{ }^{\mathrm{Mes}} \mathrm{CNC}\right) \mathrm{Fe}\left(\right.$ isobutyl) $\left(\mathrm{N}_{2}\right)(\mathrm{I})$ in $\mathrm{C}_{6} \mathrm{D}_{6}$ at $25^{\circ} \mathrm{C}$. 


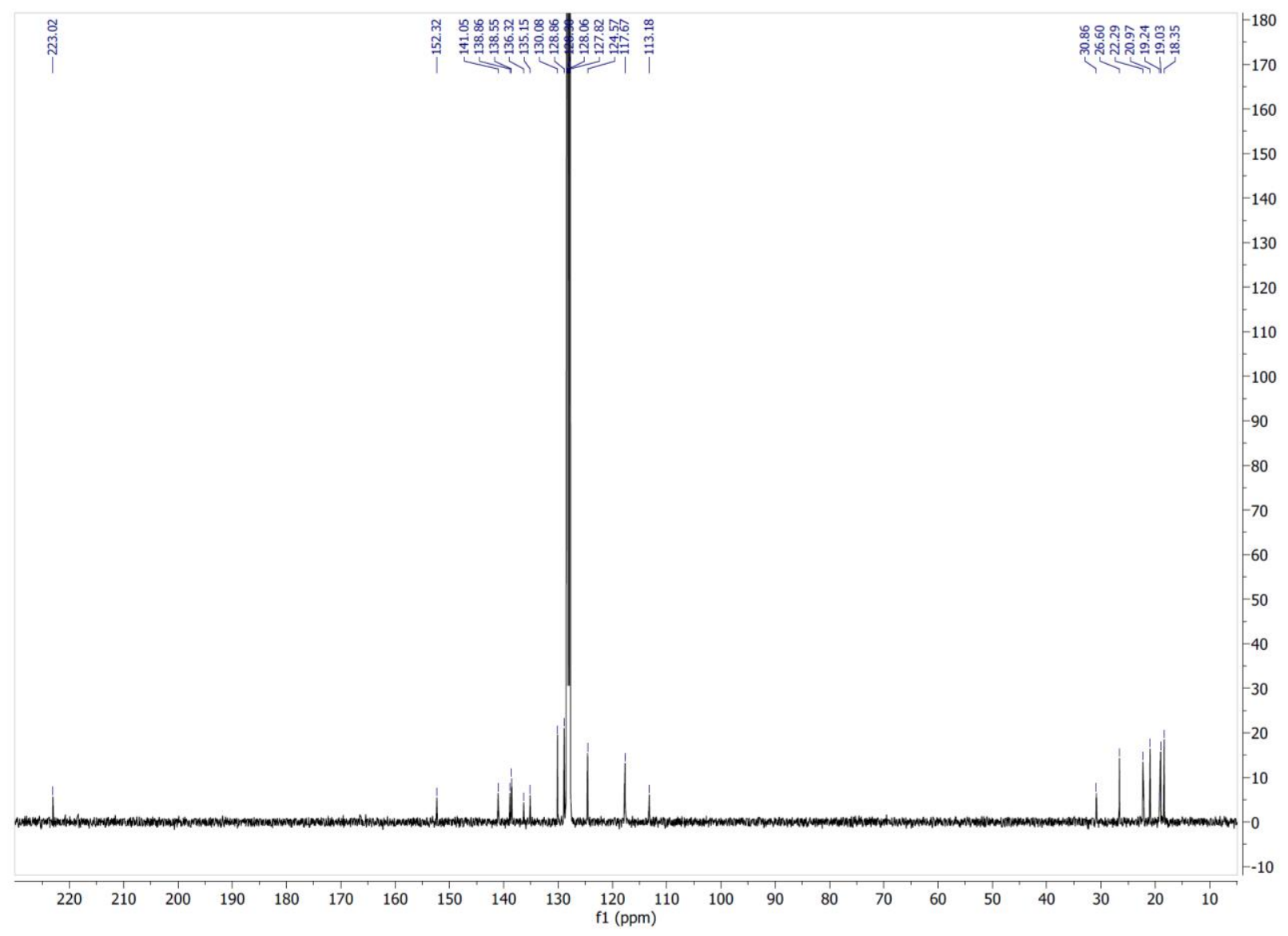

Figure S20. ${ }^{13} \mathrm{C}-\mathrm{NMR}$ spectrum of $\left(3,5-\mathrm{Me}_{2-}{ }^{\mathrm{Mes}} \mathrm{CNC}\right) \mathrm{Fe}$ (isobutyl) $\left(\mathrm{N}_{2}\right)(\mathrm{I})$ in $\mathrm{C}_{6} \mathrm{D}_{6}$ at $25^{\circ} \mathrm{C}$. 


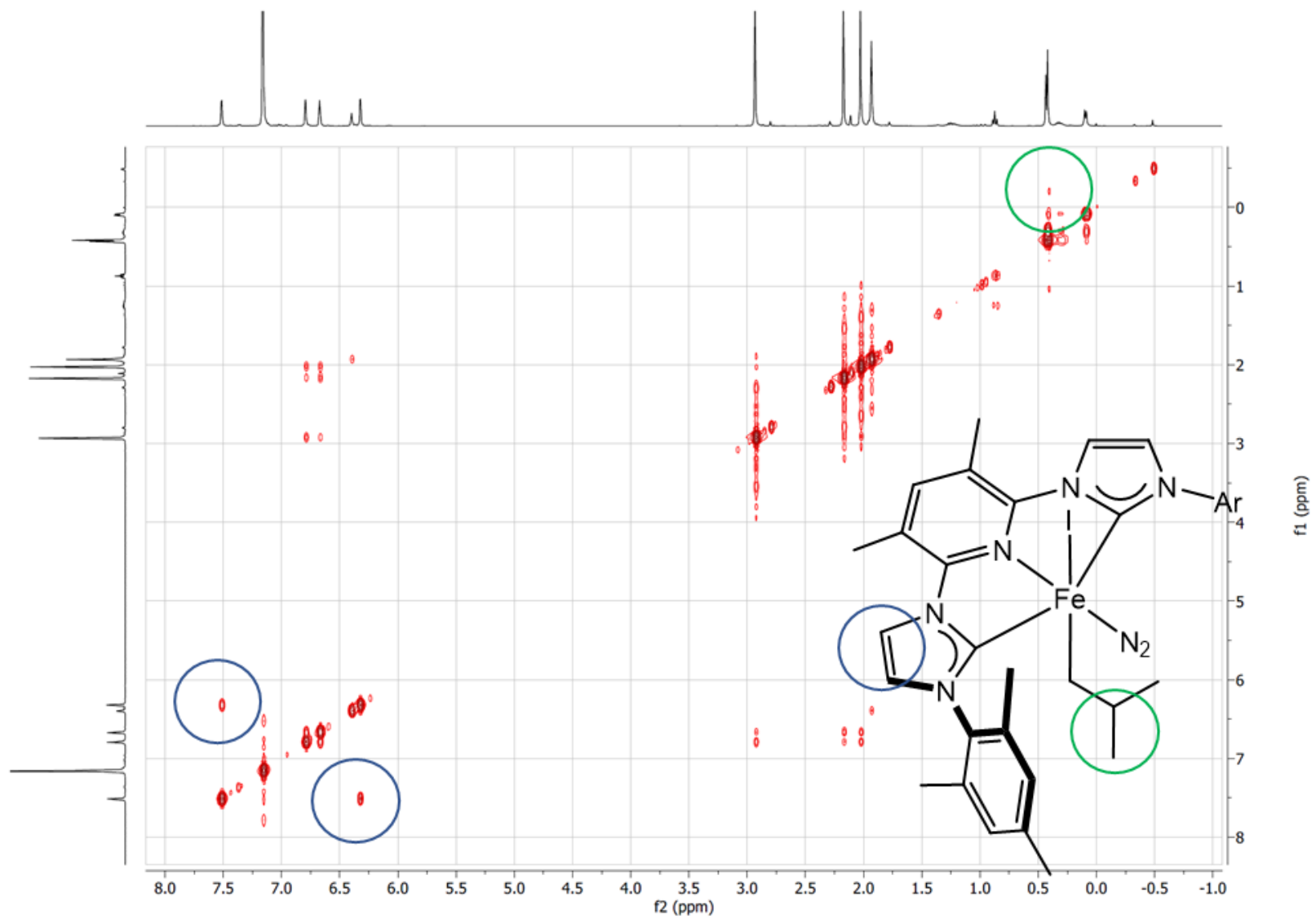

Figure S21. COSY spectrum of $\left(3,5-\mathrm{Me}_{2}{ }^{\mathrm{Mes}} \mathrm{CNC}\right) \mathrm{Fe}$ (isobutyl) $\left(\mathrm{N}_{2}\right)(\mathrm{I})$ in $\mathrm{C}_{6} \mathrm{D}_{6}$ at $25^{\circ} \mathrm{C}$. 


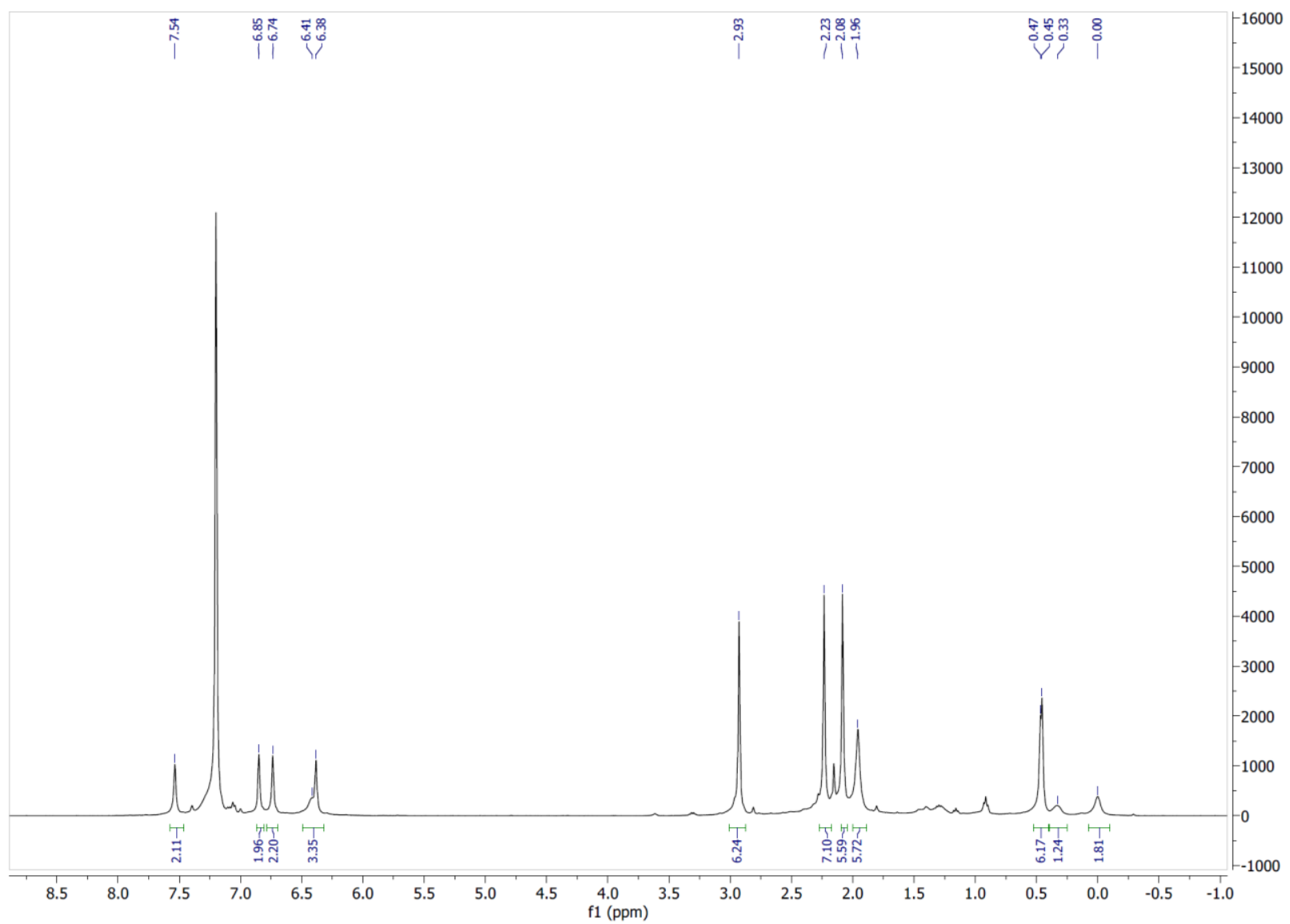

Figure S22. ${ }^{1} \mathrm{H}-\mathrm{NMR}$ spectrum of $\left(3,5-\mathrm{Me}_{2-}{ }^{\mathrm{Mes}} \mathrm{CNC}\right) \mathrm{Fe}$ (isobutyl) $\left(\mathrm{N}_{2}\right)(\mathrm{Br})$ in $\mathrm{C}_{6} \mathrm{D}_{6}$ at $25{ }^{\circ} \mathrm{C}$. 


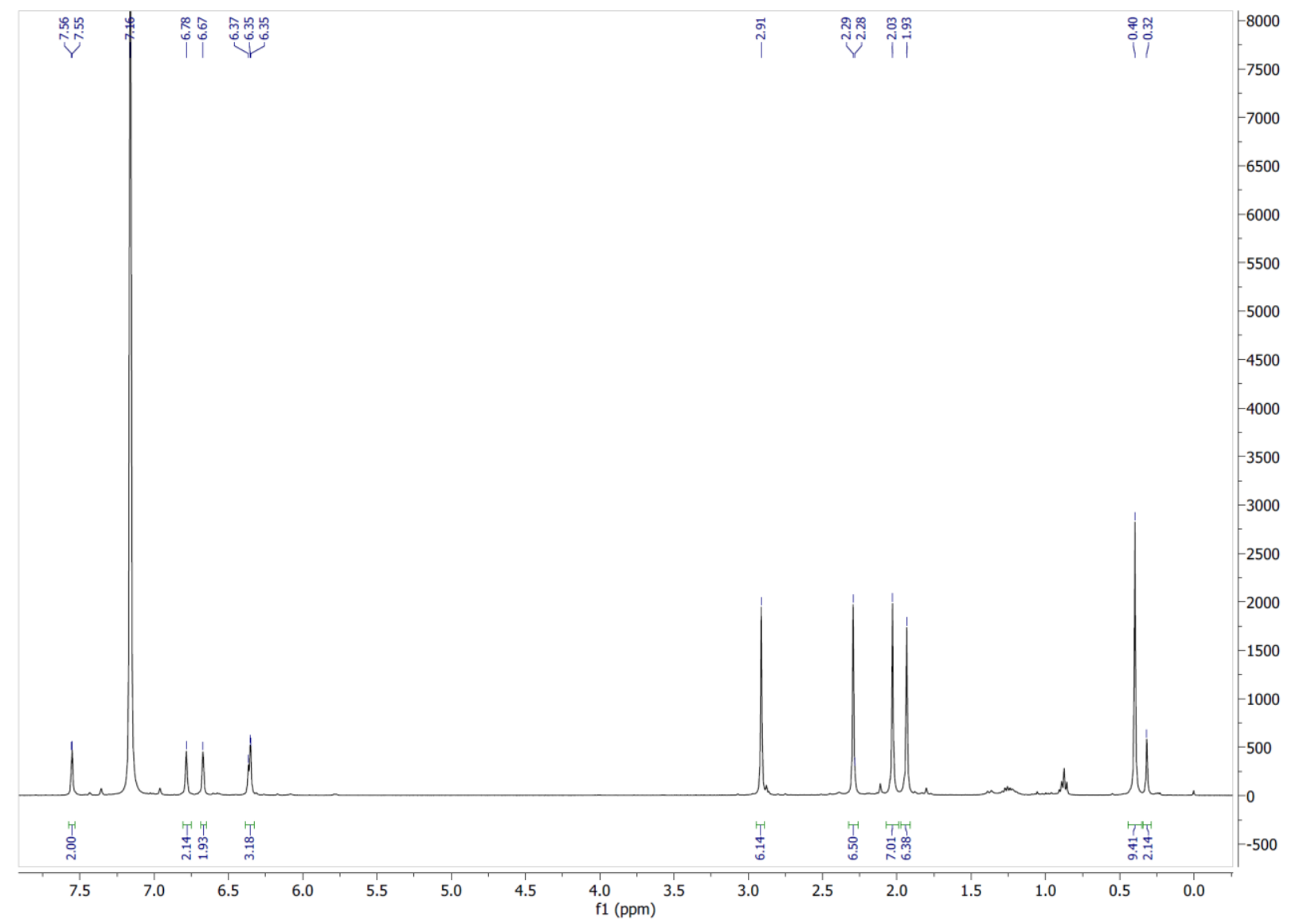

Figure S23. ${ }^{1} \mathrm{H}-\mathrm{NMR}$ spectrum of $\left(3,5-\mathrm{Me}_{2-}{ }^{\mathrm{Mes}} \mathrm{CNC}\right) \mathrm{Fe}($ neopentyl $)\left(\mathrm{N}_{2}\right)(\mathrm{I})$ in $\mathrm{C}_{6} \mathrm{D}_{6}$ at $25^{\circ} \mathrm{C}$. 


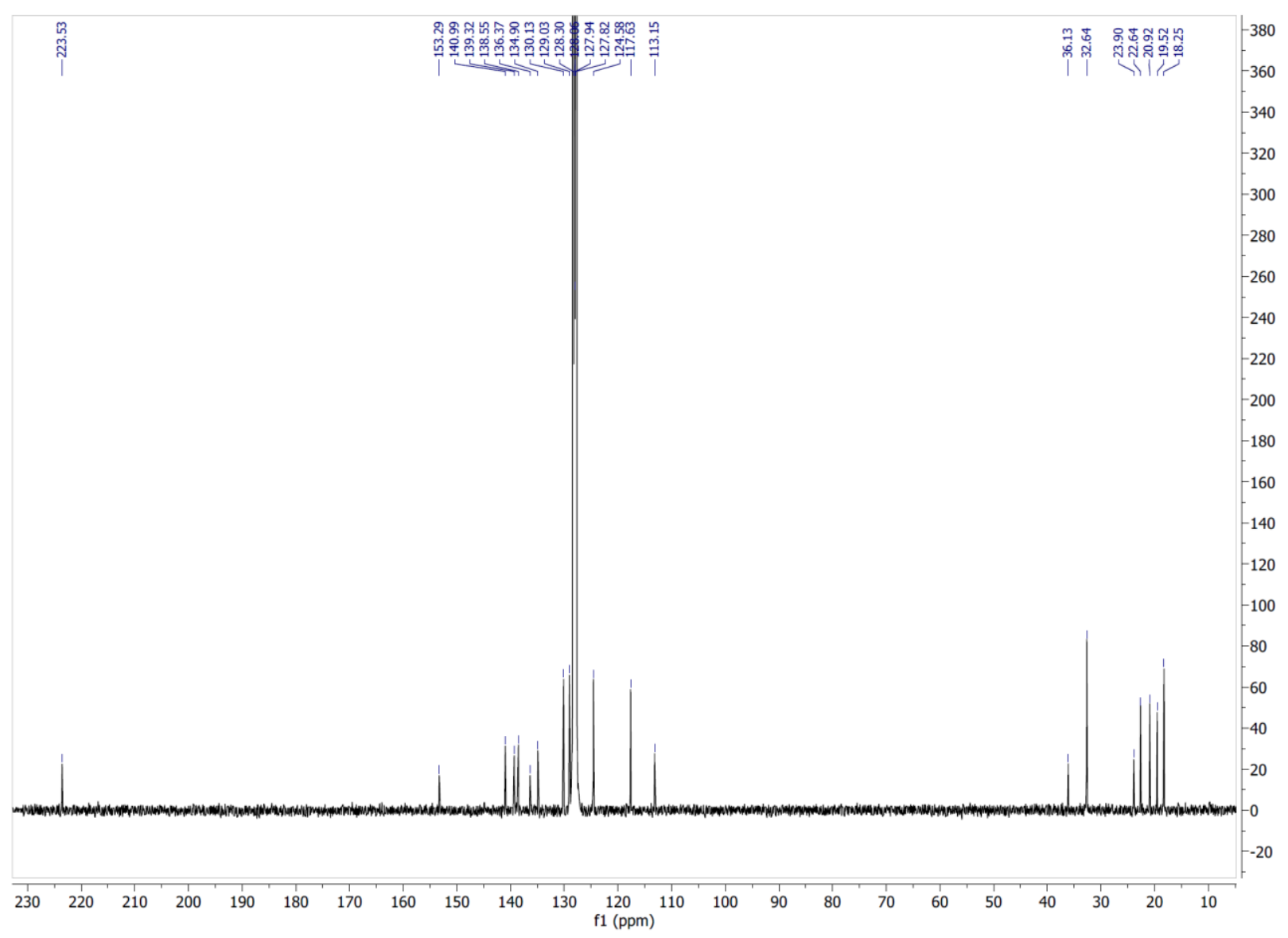

Figure S24. ${ }^{13} \mathrm{C}-\mathrm{NMR}$ spectrum of $\left(3,5-\mathrm{Me}_{2}{ }^{\mathrm{Mes}} \mathrm{CNC}\right) \mathrm{Fe}\left(\right.$ neopentyl) $\left(\mathrm{N}_{2}\right)(\mathrm{I})$ in $\mathrm{C}_{6} \mathrm{D}_{6}$ at $25{ }^{\circ} \mathrm{C}$. 


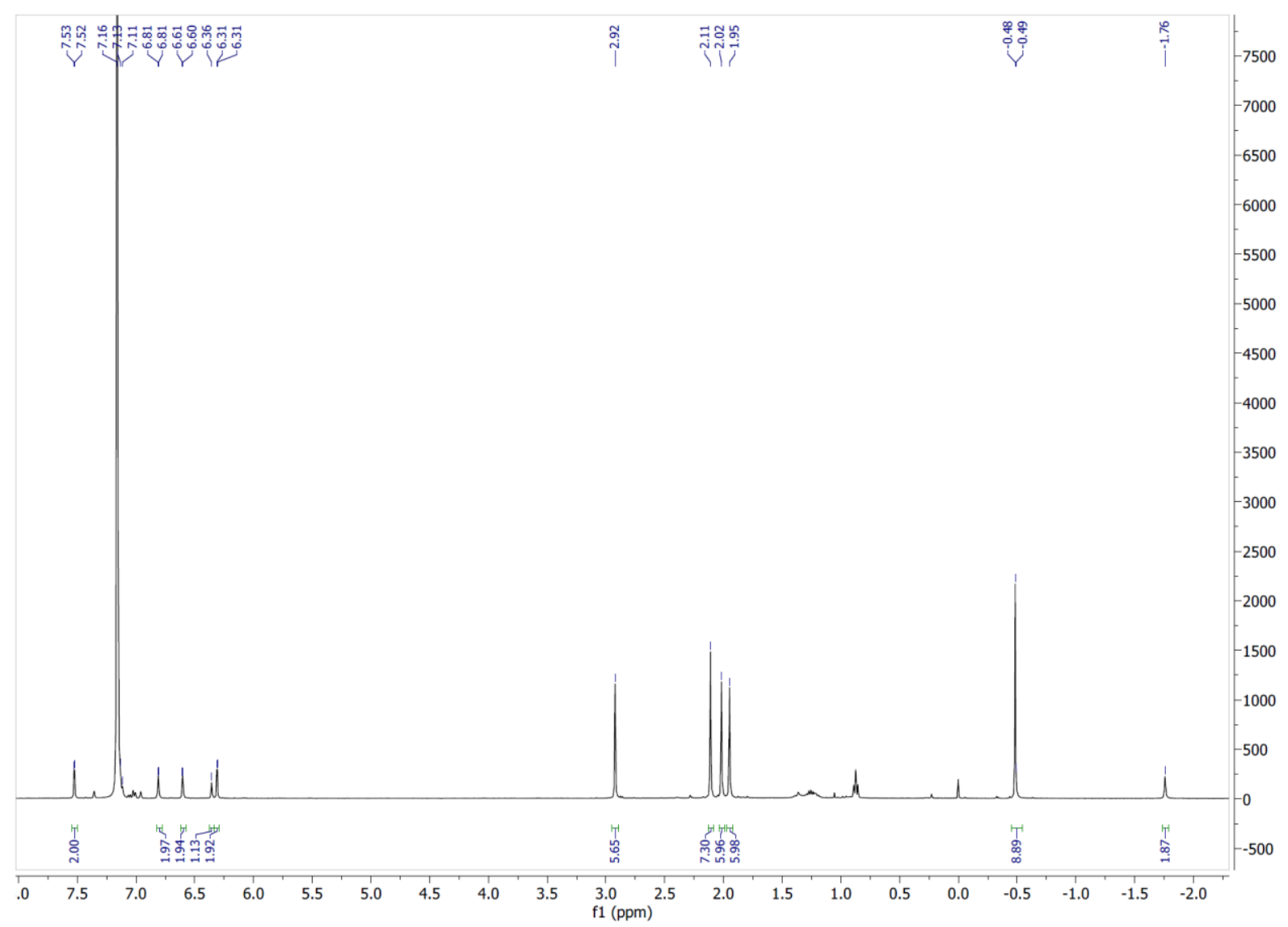

Figure S25. ${ }^{1} \mathrm{H}-\mathrm{NMR}$ spectrum of $\left(3,5-\mathrm{Me}_{2}{ }^{\mathrm{Mes}} \mathrm{CNC}\right) \mathrm{Fe}\left(\right.$ neosilyl) $\left(\mathrm{N}_{2}\right)(\mathrm{I})$ in $\mathrm{C}_{6} \mathrm{D}_{6}$ at $25^{\circ} \mathrm{C}$. 


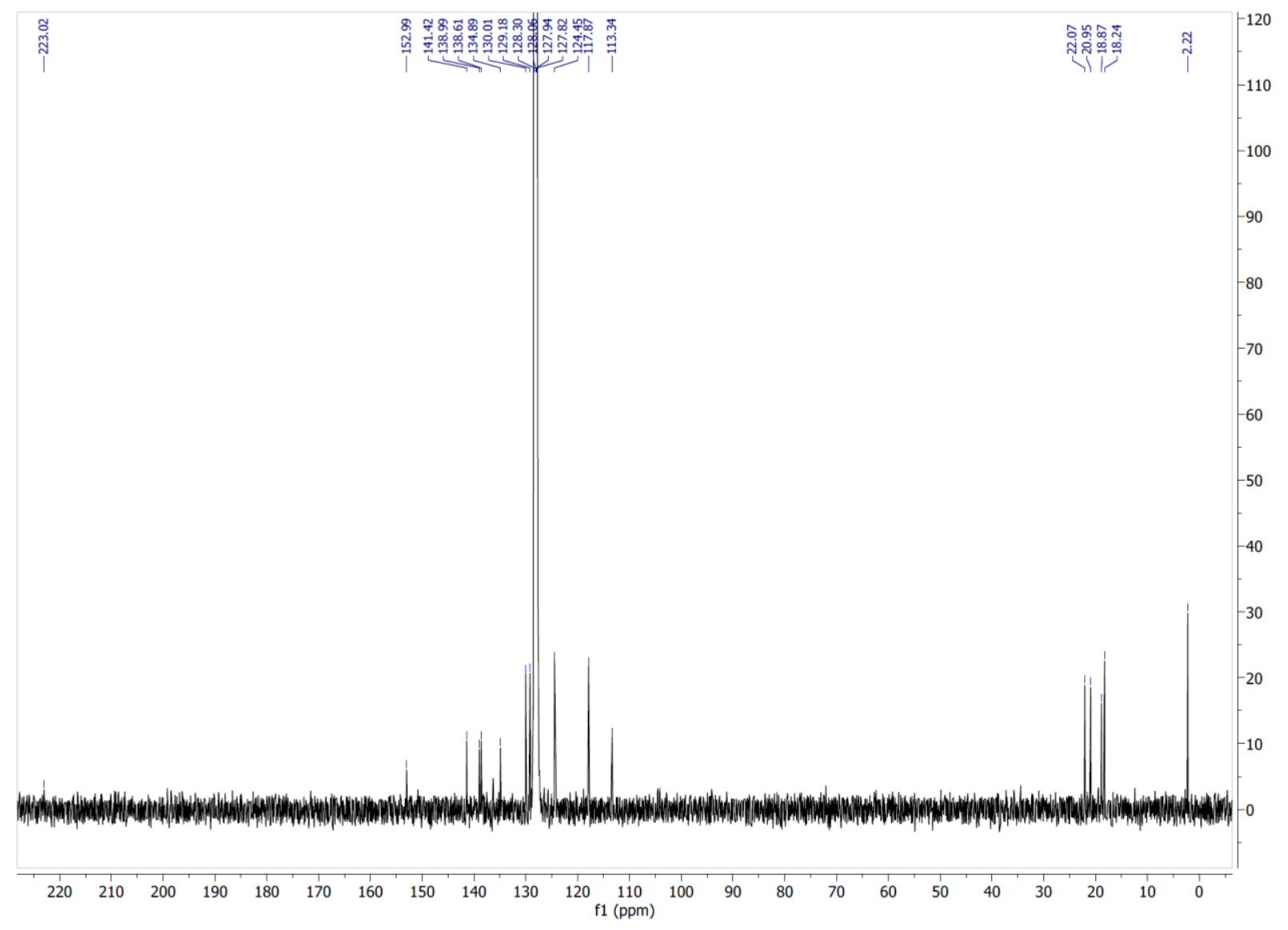

Figure S26. ${ }^{13} \mathrm{C}-\mathrm{NMR}$ spectrum of $\left(3,5-\mathrm{Me}_{2}{ }^{\mathrm{Mes}} \mathrm{CNC}\right) \mathrm{Fe}\left(\right.$ neosilyl) $\left(\mathrm{N}_{2}\right)(\mathrm{I})$ in $\mathrm{C}_{6} \mathrm{D}_{6}$ at $25^{\circ} \mathrm{C}$. 


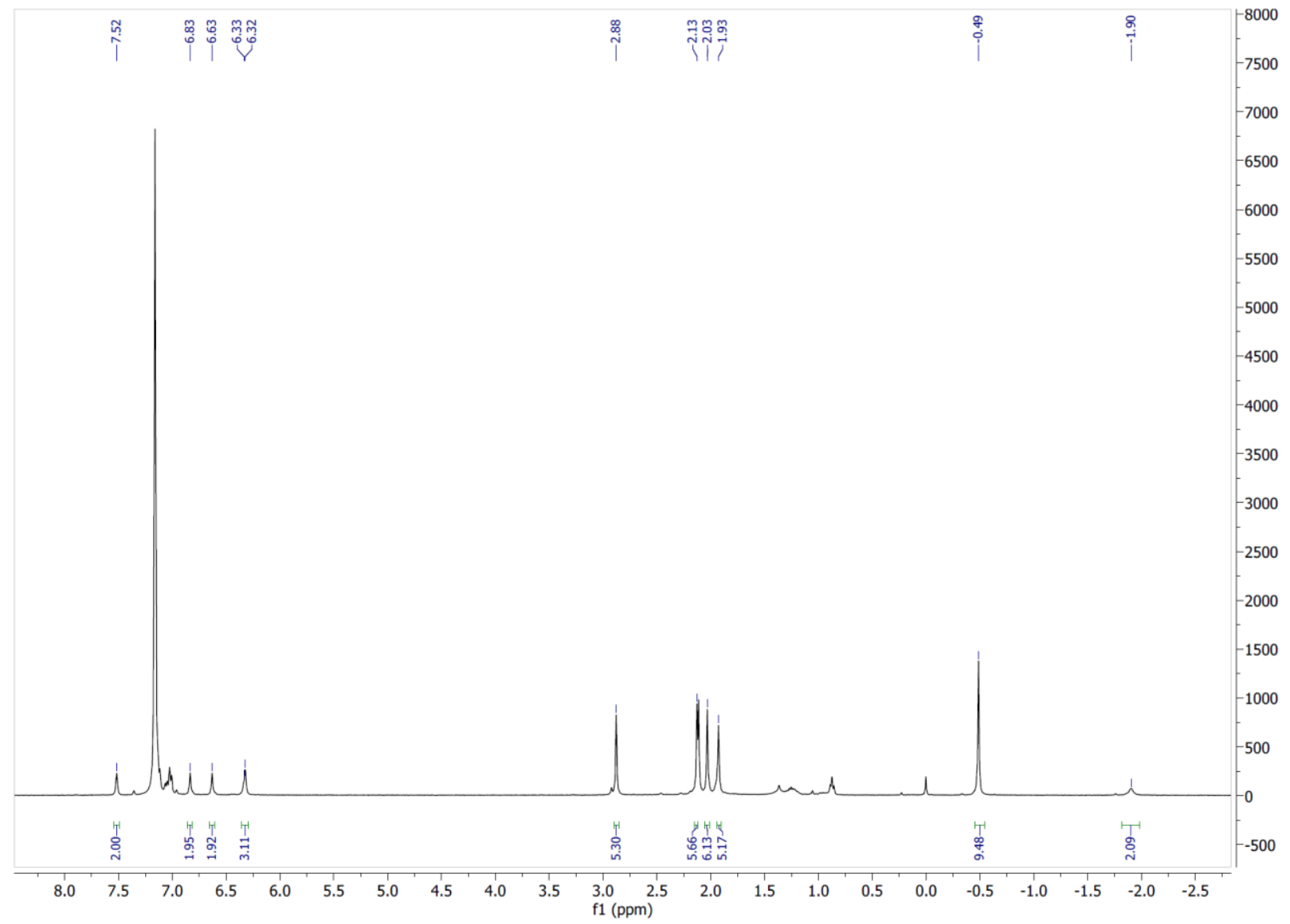

Figure S27. ${ }^{1} \mathrm{H}-\mathrm{NMR}$ spectrum of $\left(3,5-\mathrm{Me}_{2}{ }^{\mathrm{Mes}} \mathrm{CNC}\right) \mathrm{Fe}\left(\right.$ neosilyl) $\left(\mathrm{N}_{2}\right)(\mathrm{Br})$ in $\mathrm{C}_{6} \mathrm{D}_{6}$ at $25^{\circ} \mathrm{C}$. 


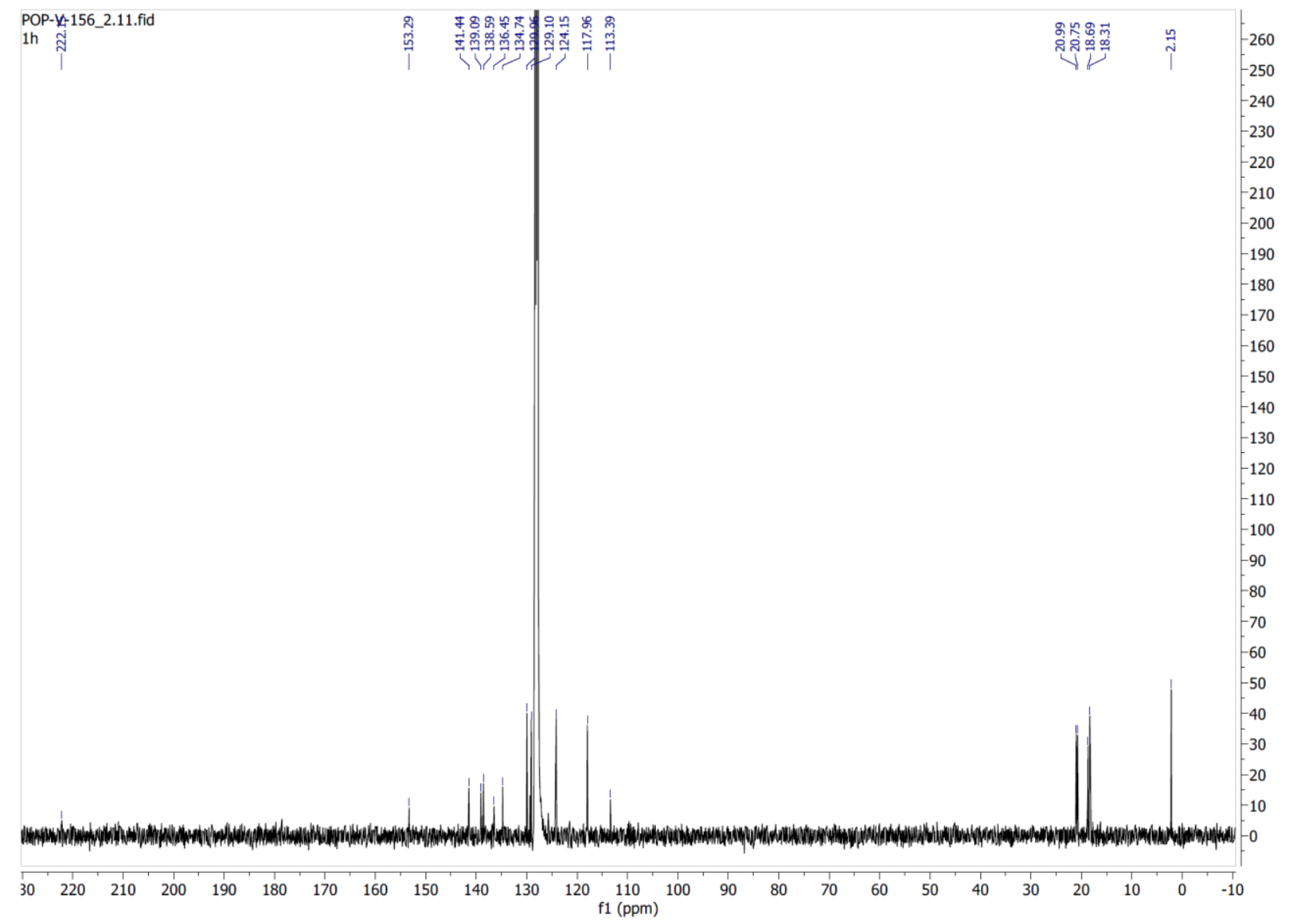

Figure S28. ${ }^{13} \mathrm{C}-\mathrm{NMR}$ spectrum of $\left(3,5-\mathrm{Me}_{2}-{ }^{\mathrm{Mes}} \mathrm{CNC}\right) \mathrm{Fe}($ neosilyl $)\left(\mathrm{N}_{2}\right)(\mathrm{Br})$ in $\mathrm{C}_{6} \mathrm{D}_{6}$ at $25^{\circ} \mathrm{C}$. 


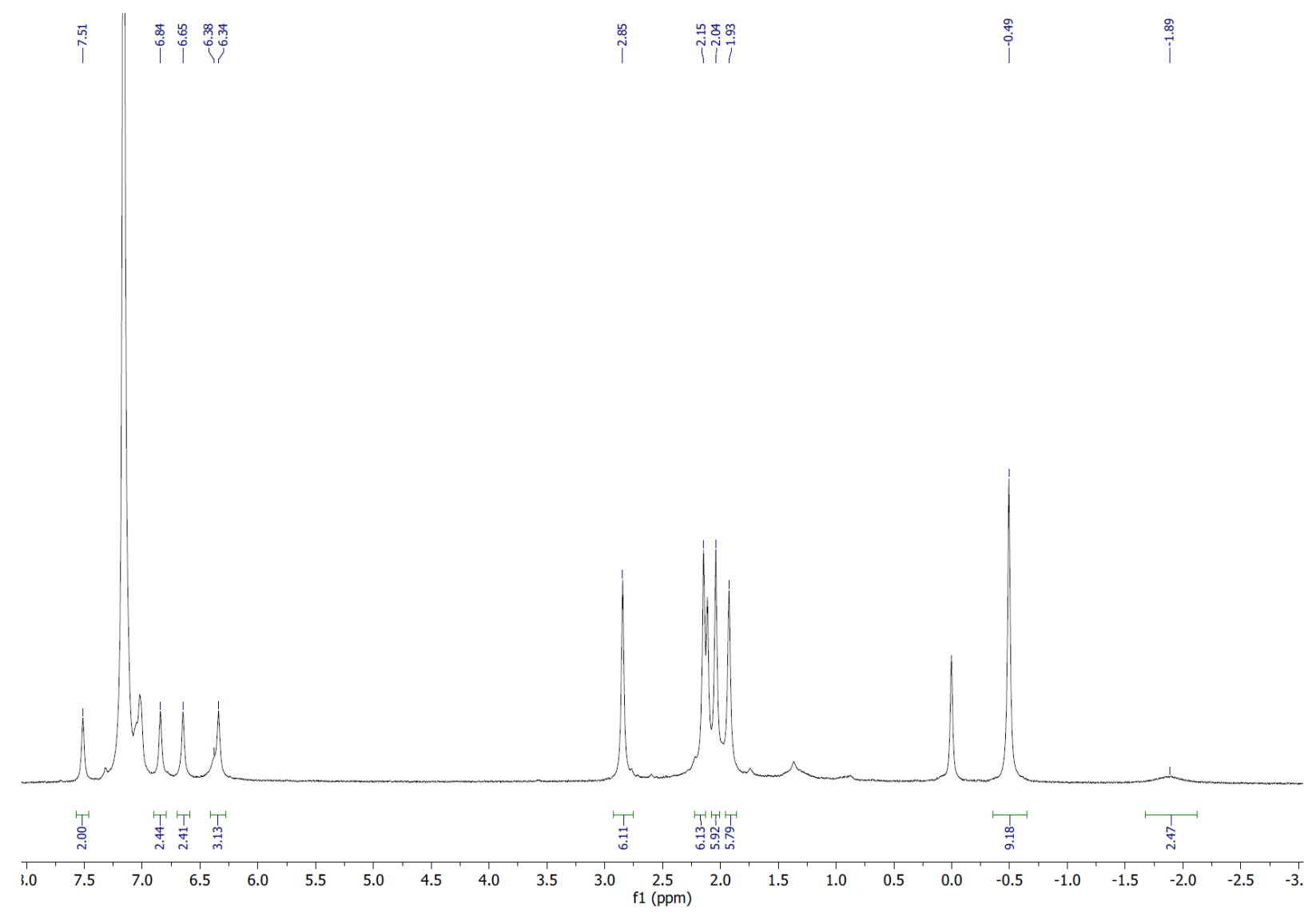

Figure S29. ${ }^{1} \mathrm{H}-\mathrm{NMR}$ spectrum of $\left(3,5-\mathrm{Me}_{2-}{ }^{\mathrm{Mes}} \mathrm{CNC}\right) \mathrm{Fe}($ neosilyl $)\left(\mathrm{N}_{2}\right)(\mathrm{Cl})$ in $\mathrm{C}_{6} \mathrm{D}_{6}$ at $25^{\circ} \mathrm{C}$. 


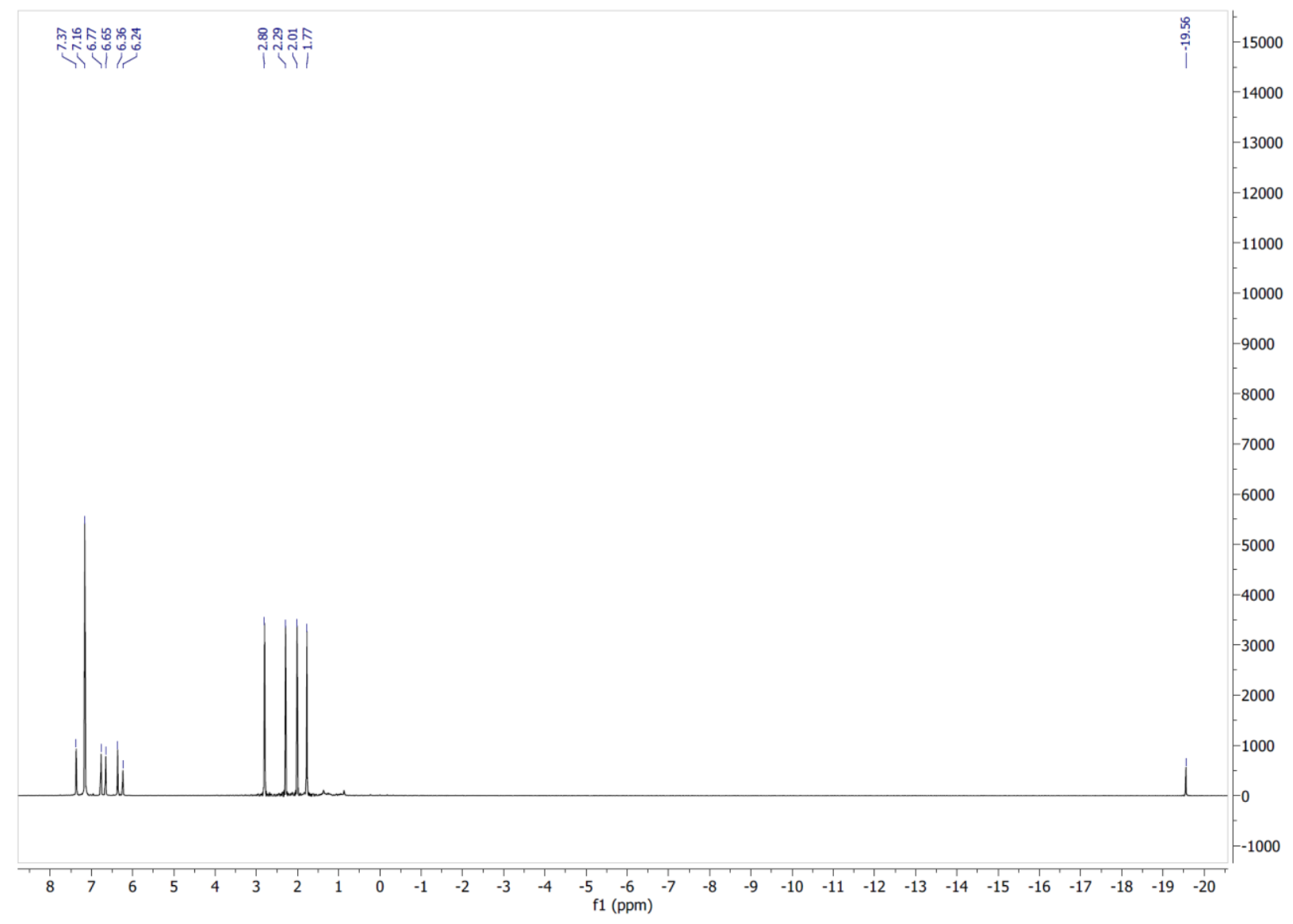

Figure S30. ${ }^{1} \mathrm{H}-\mathrm{NMR}$ spectrum of $\left(3,5-\mathrm{Me}_{2}{ }^{\mathrm{Mes}} \mathrm{CNC}\right) \mathrm{Fe}(\mathrm{H})\left(\mathrm{N}_{2}\right)(\mathrm{I})$ in $\mathrm{C}_{6} \mathrm{D}_{6}$ at $25^{\circ} \mathrm{C}$. 


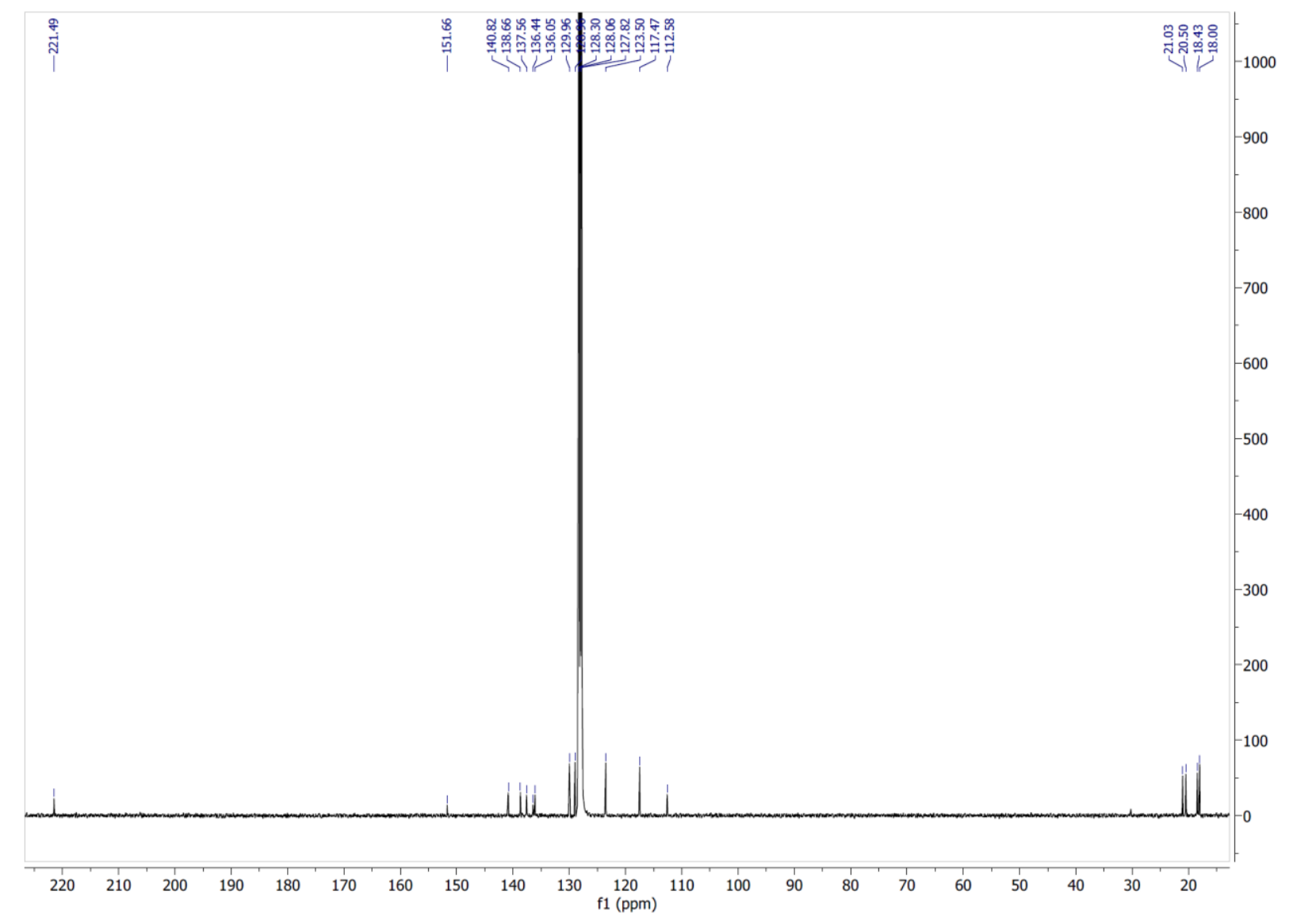

Figure S31. ${ }^{13} \mathrm{C}-\mathrm{NMR}$ spectrum of $\left(3,5-\mathrm{Me}_{2}-{ }^{\mathrm{Mes}} \mathrm{CNC}\right) \mathrm{Fe}(\mathrm{H})\left(\mathrm{N}_{2}\right)(\mathrm{I})$ in $\mathrm{C}_{6} \mathrm{D}_{6}$ at $25^{\circ} \mathrm{C}$. 


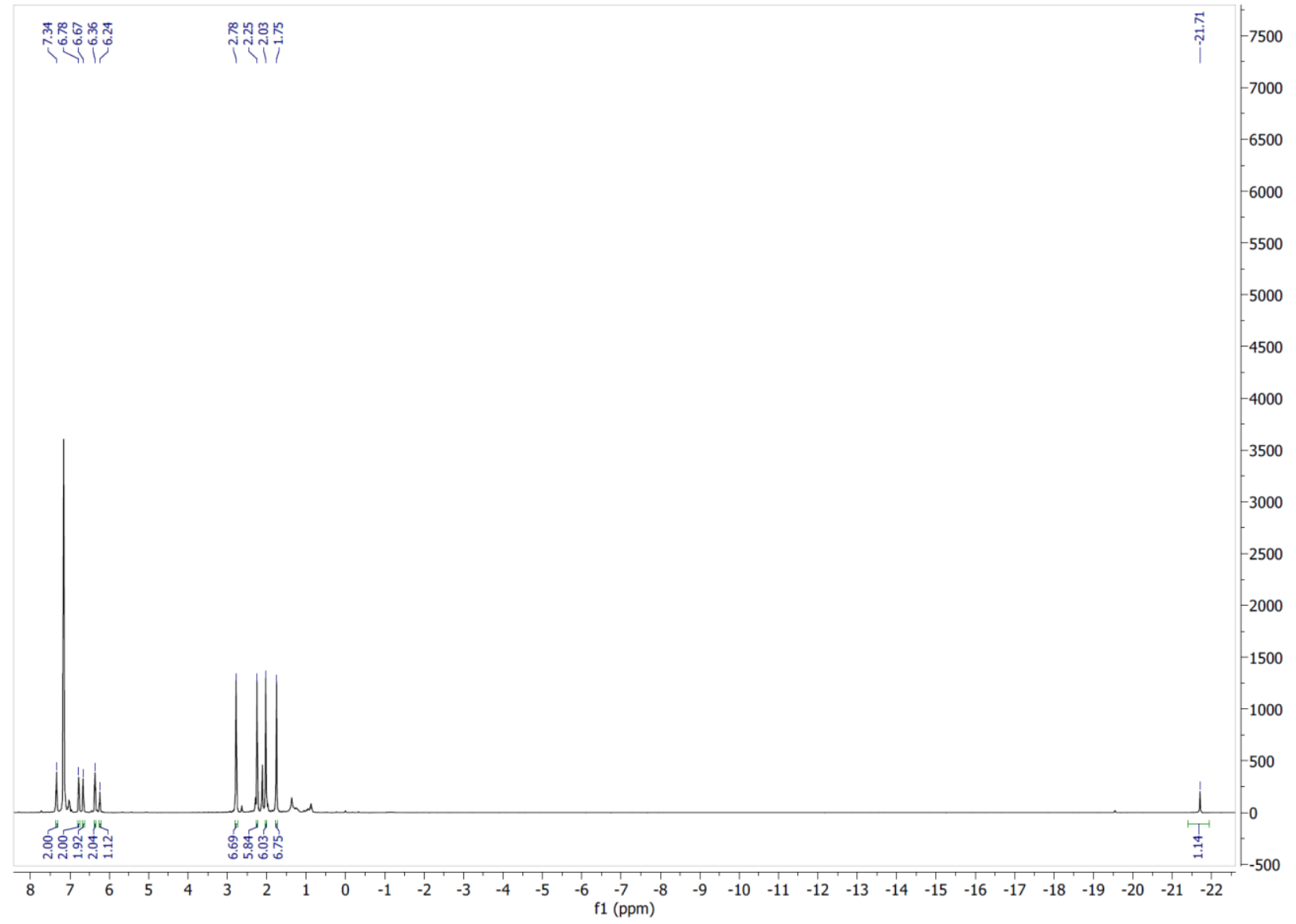

Figure S32. ${ }^{1} \mathrm{H}-\mathrm{NMR}$ spectrum of $\left(3,5-\mathrm{Me}_{2}{ }^{\mathrm{Mes}} \mathrm{CNC}\right) \mathrm{Fe}(\mathrm{H})\left(\mathrm{N}_{2}\right)(\mathrm{Br})$ in $\mathrm{C}_{6} \mathrm{D}_{6}$ at $25^{\circ} \mathrm{C}$. 


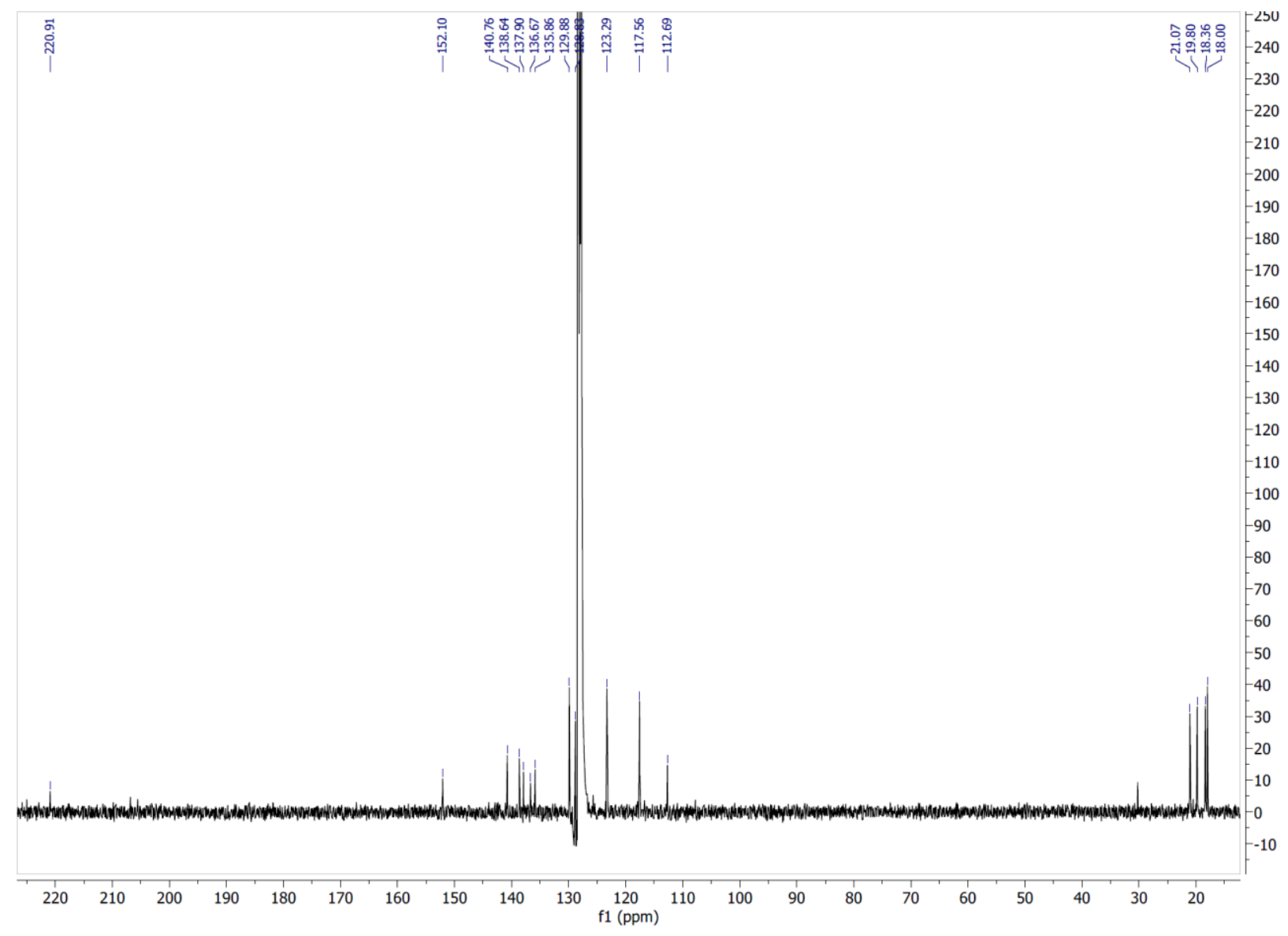

Figure S33. ${ }^{13} \mathrm{C}-\mathrm{NMR}$ spectrum of $\left(3,5-\mathrm{Me}_{2}-{ }^{\mathrm{Mes}} \mathrm{CNC}\right) \mathrm{Fe}(\mathrm{H})\left(\mathrm{N}_{2}\right)(\mathrm{Br})$ in $\mathrm{C}_{6} \mathrm{D}_{6}$ at $25^{\circ} \mathrm{C}$. 


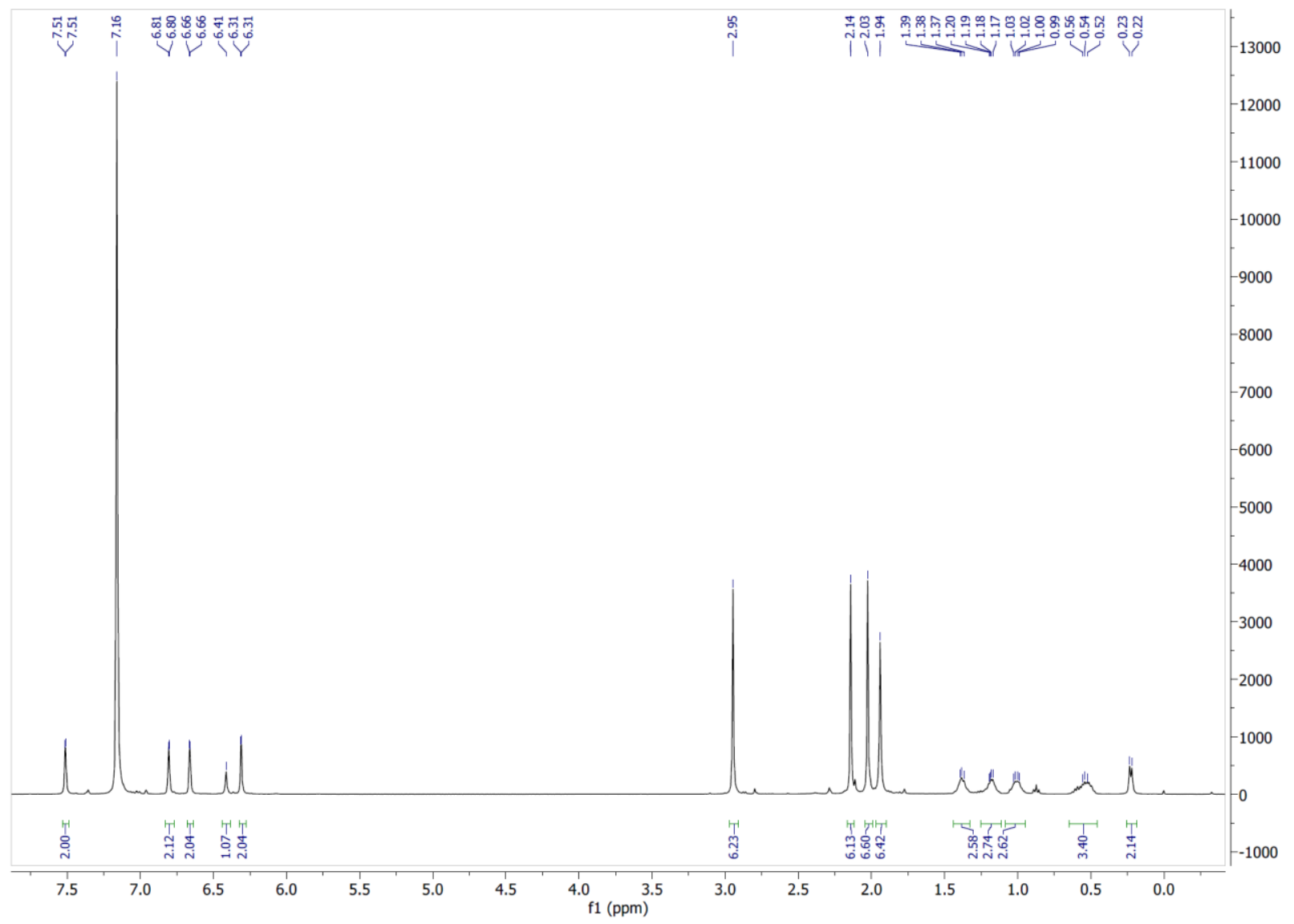

Figure S34. ${ }^{1} \mathrm{H}-\mathrm{NMR}$ spectrum of $\left(3,5-\mathrm{Me}_{2}{ }^{\mathrm{Mes}} \mathrm{CNC}\right) \mathrm{Fe}$ (methylcyclopentyl) $\left(\mathrm{N}_{2}\right)(\mathrm{I})$ in $\mathrm{C}_{6} \mathrm{D}_{6}$ at 25 ${ }^{\circ} \mathrm{C}$. 


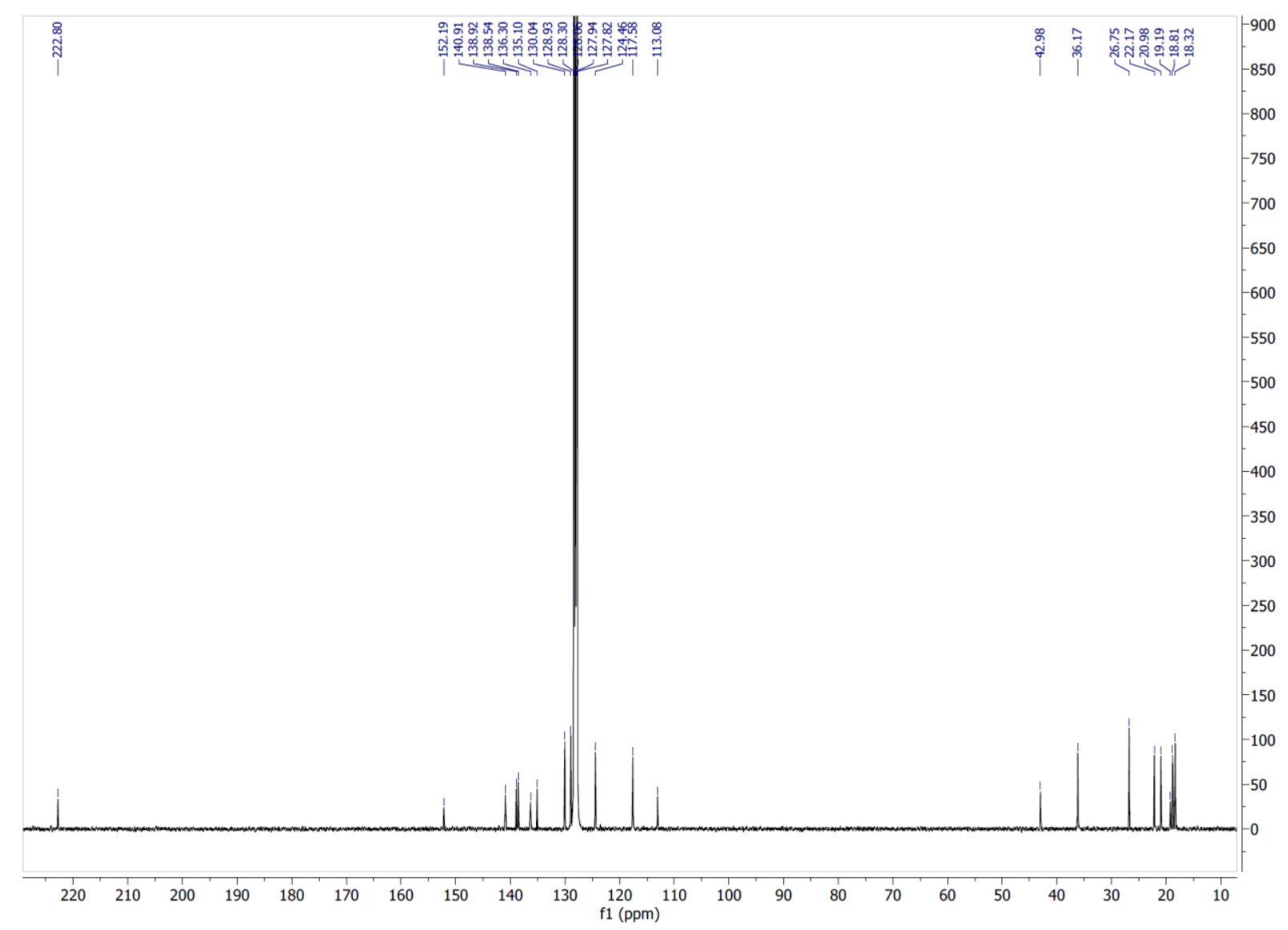

Figure S35. ${ }^{13} \mathrm{C}-\mathrm{NMR}$ spectrum of $\left(3,5-\mathrm{Me}_{2}{ }^{\mathrm{Mes}} \mathrm{CNC}\right) \mathrm{Fe}$ (methylcyclopentyl) $\left(\mathrm{N}_{2}\right)(\mathrm{I})$ in $\mathrm{C}_{6} \mathrm{D}_{6}$ at 25 ${ }^{\circ} \mathrm{C}$. 


\section{References}

1. Pangborn, A. B.; Giardello, M. A.; Grubbs, R. H.; Rosen, R. K.; Timmers, F. J. Safe and Convenient Procedure for Solvent Purification. Organometallics 1996, 15, 1518-1520.

2. Rummelt, S. M.; Darmon, J. M.; Yu, R. P.; Viereck, P.; Pabst, T. P.; Turner, Z. R.; Margulieux, G. W.; Gu, S.; Chirik, P. J. Synthesis, Structure, and Hydrogenolysis of Pyridine Dicarbene Iron Dialkyl Complexes. Organometallics 2019, 38, 3159-3168.

3. Ion Prisecaru, WMOSS4 Mössbauer Spectral Analysis Software, www.wmoss.org, 20092016.

4. Igau, A; Gladysz, J. A.; Reactions of the neohexyl iodide complex [( $\eta^{5}-$ $\left.\left.\mathrm{C}_{5} \mathrm{H}_{5}\right) \mathrm{Re}(\mathrm{NO})\left(\mathrm{PPh}_{3}\right)\left(\mathrm{ICH}_{2} \mathrm{CH}_{2} \mathrm{C}\left(\mathrm{CH}_{3}\right)_{3}\right)\right]^{+} \mathrm{BF}_{4}^{-}$and Nucleophiles: Stereochemistry of Carbon-lodine Bond Cleavage in Highly Accelerated $\mathrm{S}_{\mathrm{N}} 2$ reactions. Organometallics, 1991, 10, 2327-2334. 